\title{
Wave turbulence theory of elastic plates
}

\author{
Gustavo Düring ${ }^{1}$, Christophe Josserand ${ }^{2}$, and Sergio Rica ${ }^{3,4}$ \\ Facultad de Física, Pontificia Universidad Católica de Chile, Casilla 306, Santiago, Chile \\ ${ }^{2}$ Sorbonne Universits, CNRS \& UPMC Univ Paris 06, UMR 7190, Institut d'Alembert, F-75005, Paris, France \\ ${ }^{3}$ Facultad de Ingeniería y Ciencias, Universidad Adolfo Ibáñez, Avda. Diagonal las Torres 2640, Peñalolén, Santiago, Chile. \\ 4 UAI Physics Center, Universidad Adolfo Ibáñez, Santiago, Chile.
}

\begin{abstract}
This article presents the complete study of the long-time evolution of random waves of a vibrating thin elastic plate in the limit of small plate deformation so that modes of oscillations interact weakly. According to the wave turbulence theory a nonlinear wave system evolves in longtime creating a slow redistribution of the spectral energy from one mode to another. We derive step by step, following the method of cumulants expansion and multiscale asymptotic perturbations, the kinetic equation for the second order cumulants as well as the second and fourth order renormalization of the dispersion relation of the waves. We characterize the non-equilibrium evolution to an equilibrium wave spectrum, which happens to be the well known RayleighJeans distribution. Moreover we show the existence of an energy cascade, often called the Kolmogorov-Zakharov spectrum, which happens to be not simply a power law, but a logarithmic correction to the Rayleigh-Jeans distribution. We perform numerical simulations confirming these scenarii, namely the equilibrium relaxation for closed systems and the existence of an energy cascade wave spectrum. Both show a good agreement between theoretical predictions and numerics. We show also some other relevant features of vibrating elastic plates, such as the existence of a self-similar wave action inverse cascade which happens to blow-up in finite time. We discuss the mechanism of the wave breakdown phenomena in elastic plates as well as the limit of strong turbulence which arises as the thickness of the plate vanishes. Finally, we discuss the role of dissipation and the connection with experiments, and the generalization of the wave turbulence theory to elastic shells.
\end{abstract}

\section{Introduction}

Since more than fifty years it was established that long-time statistical properties of randomly fluctuating wavy systems possess a natural asymptotic closure because of the dispersive nature of the waves and the weakly nonlinear wave interaction $[1,2]$. This so-called wave (or weak) turbulence theory (noted WTT later on) has proven to be a powerful method to study the evolution of nonlinear dispersive wave systems $[3,4,5,6]$. As a main result, WTT predicts that the longtime dynamics is driven by a kinetic equation for the distribution of spectral densities. This method was first developed for surface gravity waves $[1,7]$, then for plasma waves [8], surface capillary waves [9] and nonlinear optics [10, 11, 12] among others.

The resulting kinetic equation has non-equilibrium properties similar to the usual Boltzmann equation for dilute gases, conserving the energy and the momentum. Moreover, it exhibits a H-theorem driving an isolated system towards equilibrium, characterized by the so-called Rayleigh-Jeans distribution. Most importantly, besides the elementary equilibrium (or thermodynamic) solution, Zakharov has shown [8] that non-equilibrium stationary solutions also arise which describe a constant flux transfer (or cascade) of a conserved quantities (e.g. energy) between large and small length scales. In the particular cases where these cascade solutions are power laws, they are named Kolmogorov-Zakharov (KZ) spectra.

Experimental evidences of $\mathrm{KZ}$ spectra have been found for ocean surface waves [13] and for capillary surfaces waves $[14,15,16]$. On the other hand, numerical simulations of surface waves have exhibited KZ spectrum for weak turbulent capillary waves [17] and, more recently, for gravity waves [18].

Ten years ago, we have shown using WTT that weak wave turbulence was also possible for elastic plates since they exhibit dispersive linear waves [19]. Indeed, adding inertia to the well known (static) theory of thin plates, one finds ballistic dispersive waves [20], which interact via cubic nonlinear terms that are weak if the plate deformations are small. Because of this cubic nonlinearity, the wave interaction mechanism involves four wave resonances and the kinetic equation has been deduced using the classical WTT framework. As a consequence, in addition to the Rayleigh-Jeans equilibrium distribution, the existence of spectra of direct energy cascade was proven theoretically and observed numerically. 
Soon after our original work, elastic plate wave turbulence was observed experimentally, showing slightly different spectrum power laws [21, 22]. Following these discoveries, numerous works have investigated the dynamics of oscillating elastic plates to explain this discrepancy between numerics-theory and experiments in the turbulent spectra [23, 24, 25, 26, 27, 28, 29], showing eventually that it could be mainly attributed to the particular dissipation of real plates.Vibrating elastic plates have been shown to provide an excellent prototype system to investigate and test different wave turbulence regimes, such as inverse cascade [30], transitory dynamics $[29,31,32,33]$ high forcing [34, 35], the breakdown of the WTT or the onset of intermittency [36, 37, 38] for instance.

The goal of the present paper is to present a full and consistent derivation of the wave turbulence theory for elastic plates in different configurations, from the planar plates to the cylindrical or spherical shells. First, we develop the general wave turbulence theory for the surface deflection of an elastic plate, we study the stationary solutions and its properties. More precisely, we derive step by step the kinetic equation of Ref. [19] using the WTT technique, solving the BBGKY hierarchy of differential equations for the cumulants of the Fourier amplitude of the waves via a multi-scale analysis. This general framework provides a natural asymptotic (long time) closure, that allows us to express high order cumulants in terms of the second order one. This kinetic equation results at the fourth order in the perturbative expansion of the wave amplitudes. Prior, we derive consistently the second order renormalization of the oscillation frequency for bending waves [39], experimentally observed in [26]. In addition we calculate the energy flux spectrum and explicitly verified the locality of the interaction. Then, we show numerically the relaxation to equilibrium and the existence of an energy cascade with a Kolmogorov spectra for the plate dynamics. Finally, we discuss several related topics, such as the posible existence of an inverse cascade and the effect of damping in experimental observations.

\subsection{The Föppl-von Kármán equations for a planar elastic plate.}

To model the vibration of an elastic plate, the starting point is the dynamical version of the Föppl-von Kármán (FvK hereafter) equations $[40,41,42]$ for the vertical amplitude of the deformation $\zeta(x, y, t)$ and for the Airy stress function $\chi(x, y, t)$ :

$$
\begin{aligned}
\rho \frac{\partial^{2} \zeta}{\partial t^{2}} & =-\frac{E h^{2}}{12\left(1-\sigma^{2}\right)} \Delta^{2} \zeta+\{\zeta, \chi\} \\
\frac{1}{E} \Delta^{2} \chi & =-\frac{1}{2}\{\zeta, \zeta\} .
\end{aligned}
$$

Here, $h$ is the thickness of the elastic sheet, the material has a mass density $\rho$, a Young modulus $E$ and its Poisson ratio is $\sigma$. $\Delta=\partial_{x x}+\partial_{y y}$ is the usual Laplacian and the bracket $\{\cdot, \cdot\}$ is defined by $\{f, g\} \equiv f_{x x} g_{y y}+f_{y y} g_{x x}-2 f_{x y} g_{x y}$, which is an exact divergence, so that equation (1) preserves the momentum of the center of mass, namely $\partial_{t t}\left(h \rho \int \zeta(x, y, t) d x d y\right)=0$. Equation (2) for the Airy stress function $\chi(x, y, t)$ may be seen as the compatibility equation for the in-plane stress tensor which follows the dynamics at the lowest order in the dynamics. In the derivation we have omitted the inertia of the in-plane modes of oscillations, or in other words we assume that the in-plane displacements are negligible and the quasi-static force balance holds, so that, as said, equation (2) follows the dynamics.

Small plane waves perturbations $\left(\zeta \propto e^{i(\boldsymbol{k} \cdot \boldsymbol{x}-\omega t)}\right.$ with $\left.\boldsymbol{x}=(x, y)\right)$ of a plane plate are dispersive with the usual ballistic behavior of bending waves [20, 42]:

$$
\omega_{\boldsymbol{k}}=\sqrt{\frac{E h^{2}}{12\left(1-\sigma^{2}\right) \rho}}|\boldsymbol{k}|^{2} .
$$

The nonlinearities modify this picture resulting in a nonlinear wave equation: deducing the "formal" solution of $\chi$ from equation (2) and inverting the bi-laplacian, one gets formally

$$
\chi=-\frac{E}{2} \Delta^{-2}\{\zeta, \zeta\}
$$

When introduced into (1), it yields:

$$
\rho \frac{\partial^{2} \zeta}{\partial t^{2}}=-\frac{E h^{2}}{12\left(1-\sigma^{2}\right)} \Delta^{2} \zeta-\frac{E}{2}\left\{\zeta, \Delta^{-2}\{\zeta, \zeta\}\right\},
$$

that can be interpeted as a cubic nonlinear wave equation for the amplitude of deformation. 
The total mechanical energy of the elastic plate is conserved by the dynamics of equation (1) and (2). This energy includes the kinetic part, the bending part, and the contribution for the stretching coming from the in-plane stresses induced by the amplitude deformations [42]:

$$
H[\zeta, \chi]=h \int\left(\frac{\rho}{2} \dot{\zeta}^{2}+\frac{E h^{2}}{24\left(1-\sigma^{2}\right)}(\Delta \zeta)^{2}-\frac{1}{2 E}(\Delta \chi)^{2}-\frac{1}{2} \chi\{\zeta, \zeta\}\right) d \boldsymbol{r} .
$$

As before, using (4) and integrating by parts, it simplifies to:

$$
H[\zeta, \chi]=h \int\left(\frac{\rho}{2} \dot{\zeta}^{2}+\frac{E h^{2}}{24\left(1-\sigma^{2}\right)}(\Delta \zeta)^{2}+\frac{E}{8}\left[\Delta^{-1}\{\zeta, \zeta\}\right]^{2}\right) d \boldsymbol{r}
$$

A first consequence of this type of writing is that the energy contains two quadratic contributions: the kinetic and the bending energy, and a quartic one corresponding to the nonlinear term: the stretching energy. More importantly, each term is positive bounding the total energy from below, witnessing a "defocusing behavior" of the wave dynamics. By "defocusing behavior" we mean that the energy contributions of bending and stretching are both positive, therefore the system cannot increase bending and stretching simultaneously because of energy conservation. Even more, since the energy is bounded from below and because of the energy conservation, none of these energies can diverge.

\subsection{Wave Turbulence}

The Föppl-von Kármán equations (1,2) are conservative equations which may be derived from a variational argument. However, in nature the energy is strongly dissipated through different damping mechanisms and equilibrium solutions are possible to achieve only in numerical simulations a priori. More interesting is to look for out-of-equilibrium stationary solutions under a constant energy injection and dissipation which can be modeled by adding an injection and a dissipation term in equation (1). Usually the energy injection takes place at large scales while the dissipation is concentrated at small scales. If the injection scale is well separated from the dissipation scale, an inertial range exists in which the conservative FvK equations (1,2) hold and are responsible for the energy transport from the large scales toward the dissipation scale. Such solutions are called turbulent in analogy with hydrodynamic turbulence and represent the main interest and motivation of the present work. A fundamental and common assumption in the search for turbulent solutions is the non-dependence of the dissipation and injection mechanism on the spectrum in the inertial range. Therefore in the following analytical work, we will not consider the details of the energy injection and dissipation that will be simply modeled as a net energy flux between scales. However, dissipation in elastic plates have various origins and may eventually play an influence on the experimentally observed spectra: dissipation at the boundaries, air entrainment, sound emission, viscoelastic flows or heat transfer, etc. Among all these mechanisms, sound radiation, air entrainment and heat losses are weak effect and can be in general neglected, while bulk viscoeslatic dissipation and bending waves radiation at the boundaries seem to be the dominant mechanisms, as shown in Ref. [27]. Then, the theoretical energy cascade is difficult to observe experimentally because the injection scale and the dissipation one are not well separated in the spectral available domain, as it will be discussed in section 8.1.

\subsection{Dimensional derivation of turbulent spectra}

Turbulent spectra can be discussed firstly on dimensional grounds, following the seminal work of Kolmogorov [43]. However, additional parameters are present in the case of wave turbulence due to the dispersion relation of the linear waves and the thickness of the plate. In principle, the energy spectrum does depend on the physical characteristics of the plate as the Young modulus $E$, the mass density $\rho$, the thickness $h$. Moreover, the Kolmogorov assumption tells us that the spectrum is also a function on the energy flux $P$ and the wave number $k$ or the typical length scale $\ell$.

More precisely, considering the energy per unit of mass $\mathcal{E}=H /(\rho h S)$ (here $S$ is the total surface of the plate) that has the dimension of the square of a velocity $L^{2} / T^{2}$, we obtain that the spectral density of this energy $E_{k}$, defined as

$$
\mathcal{E}=\int E_{k} d k
$$

has dimension $L^{3} / T^{2}$. Here the integral is one dimensional since it is over the wavenumber amplitude $k=|\boldsymbol{k}|$. The flux of energy per unit mass $P$ in the Fourier space is defined through :

$$
\frac{\partial}{\partial t} E_{k}=-\frac{\partial}{\partial k} P
$$


so that the dimension of $P$ is $L^{2} / T^{3}$.

Following the Kolmogorov's assumption, the turbulent spectra corresponds to a constant flux of energy per unit mass $P$, and the spectrum depends only on $E / \rho$ (which is proportional to the sound speed of elastic waves in the material), the effective thickness

$$
\ell=\frac{h}{\sqrt{3\left(1-\sigma^{2}\right)}},
$$

$P$ and $k$. In the general case one gets

$$
E_{k}=\frac{E \ell}{\rho} \Phi\left(\frac{P \ell}{(E / \rho)^{3 / 2}}, k \ell\right),
$$

where $\Phi(\cdot)$ is an unknown function of the dimensionless parameters. Obviously, this is not the only possible representation because the arbitrary choice of the independent dimensionless parameters. However, it is interesting to emphasize that, at this stage, the dimensional analysis does not provide a simple power law prediction for the energy spectrum, by contrast with the classical Kolmogorov theory for fluid turbulence. In fact, two additional parameters are playing a role here, leading to the two variables of the function $\Phi$. One parameter is usual for wave turbulence and comes from the dispersion relation, which is not present in classical fluid turbulence. However, the nonlinear interaction prescribes the number of waves interacting at the first nonlinear order, leading to an additional constraint on the power law exponent for $P$ in the turbulent spectra, so that $a$ priori a simple dimensional prediction for the wave turbulence spectra can be obtained [6]. More precisely, since the nonlinear interaction term in the FvK equation is cubic, the waves interact nonlinearly through 4-waves resonances (as it can be seen in the energy density), so that the following power-law relation holds between $E_{k}$ and $P$ :

$$
E_{k} \propto P^{1 / 3}
$$

Therefore, using eq. (8), the following prediction for the turbulent spectra is obtained:

$$
E_{k}=P^{1 / 3} \sqrt{\frac{E}{\rho}} k^{-4 / 3} \Phi_{1}(k \ell),
$$

here $\Phi_{1}$ is a function of the dimensionless parameter $k \ell$. In the present case of elastic plates, because of the existence of an additional parameter linked to the effective thickness $\ell$, the energy spectrum depends on a family of solution still involving one dimensionless parameter. This additional parameter makes a specificity of the elastic plate wave turbulence for which the wave turbulence spectra cannot be deduced by dimensional analysis alone. In fact, the derivation of this function will be done by solving exactly the kinetic equation for the wave spectrum in Section 6 .

Different or other assumptions could be made for the energy spectrum, starting from eq. (9), depending on the relevant physical mechanisms. They would lead to theoretical predictions that are worth to compare with the expected wave turbulent spectra:

- Consider first the limit where the spectrum does not depend on the effective thickness $\ell$. This case corresponds to the situation of very thin elastic bodies where the bending is negligible respecting to the stretching. In this case ridges and folds are expected [34]. In that case, if the spectrum does not depend on $\ell$, we obtain:

$$
E_{k}=\frac{E}{\rho k} \Phi_{2}\left(\frac{P}{(E / \rho)^{3 / 2} k}\right) .
$$

If we still impose the 4 -waves resonances, it gives:

$$
E_{k} \propto P^{1 / 3} \sqrt{\frac{E}{\rho}} k^{-4 / 3} .
$$

This later prediction is somehow non compatible with the other assumption that the bending waves are negligible, which explains why this spectrum is not the one that will be obtained by the WTT. 
- We could also consider a limit where the spectrum does not depend on $E / \rho$, which would indicate that the elastic property of the plate does not influence the spectrum, yielding:

$$
E_{k}=P^{2 / 3} \ell^{5 / 3} \Phi_{3}(k \ell) .
$$

or similarly

$$
E_{k}=P^{2 / 3} k^{-5 / 3} \tilde{\Phi}_{3}(k \ell) .
$$

These spectra are not compatible with the 4-waves resonances, which is consistent with the limit considered.

- Finally, in the limit where the spectrum depends neither on $E / \rho$ nor on $\ell$ one gets the well known Kolmogorov spectrum of fluid turbulence:

$$
E_{k}=C P^{2 / 3} k^{-5 / 3} \text {. }
$$

This last limit induces that the oscillation time scales do not depend on the material properties (Young modulus and mass density) and involve instead the energy flux itself, explaining why the power law dependence in $P$ is different than that of the weak turbulence. It can be formally considered as a strong turbulence regime for plates.

\subsection{Föppl-von Kármán equations in dimensionless form}

For the following, we shall use dimensionless variables, following $\zeta=\ell \zeta^{\prime}$, the in-plane position vector $\boldsymbol{x}=\ell \boldsymbol{x}^{\prime}$, the time $t=\tau t^{\prime}$, with $\tau=\sqrt{\frac{\rho}{E}} \ell$, and the Airy function $\chi=E \ell^{2} \chi^{\prime}$.Therefore the Föppl-von Kármán equations read in dimensionless form:

$$
\begin{aligned}
\frac{\partial^{2} \zeta^{\prime}}{\partial t^{\prime 2}} & =-\frac{1}{4} \Delta^{2} \zeta^{\prime}+\left\{\zeta^{\prime}, \chi^{\prime}\right\} \\
\Delta^{2} \chi^{\prime} & =-\frac{1}{2}\left\{\zeta^{\prime}, \zeta^{\prime}\right\}
\end{aligned}
$$

and the dispersion relation becomes

$$
\omega_{k}=\frac{1}{2} k^{2}
$$

For the following we will drop the primes everywhere for the sake of simplicity. It is important to notice that the resulting equations exhibit no parameters, as if all the plates were equivalent! In fact, one dimensionless parameter is hidden, so that the plate dynamics depends eventually on its dimensionless size $\Lambda=\frac{L_{\text {plate }}}{\ell}=\sqrt{3\left(1-\sigma^{2}\right)} \frac{L_{\text {plate }}}{h}$. Moreover, when injection and dissipation are added, additional dimensional parameters can be introduced.

\subsection{Plan of the paper.}

In the Section 2 we recapitulate the main results of this paper, that will be demonstrated in details in the other sections: Section 3 describes the Hamiltonian structure of the dynamics and deduces the canonical variables that diagonalize the Hamiltonian. Then section 4 presents the detailed derivation of the kinetic equation using a statistical description of the dynamics. The general properties of the kinetic equation are described in section 5, followed by the deduction of the KZ spectra (section 6). Section 7 shows the numerical validation of the theoretical predictions of the WTT for elastic plates. Finally, a physical discussion of the results is provided on section 8, comparing the theoretical predictions with the experiments and investigating the limits of the WTT and the generalization of the results to other configurations. A large appendix section is then provided at the end where the more complex and heavier calculations are written in order to make the main document more easy to read.

\section{Main results}

The main problem concerns the evolution of the wave amplitudes for different normal modes of oscillation. We will use often the variables in Fourier space, defined as

$$
\begin{aligned}
\zeta(\boldsymbol{r}, t) & =\frac{1}{2 \pi} \int \zeta_{\boldsymbol{k}}(t) e^{i \boldsymbol{k} \cdot \boldsymbol{r}} d \boldsymbol{k}, \\
\chi(\boldsymbol{r}, t) & =\frac{1}{2 \pi} \int \chi_{\boldsymbol{k}}(t) e^{i \boldsymbol{k} \cdot \boldsymbol{r}} d \boldsymbol{k} .
\end{aligned}
$$

Notice that because $\zeta(\boldsymbol{r}, t)$ and $\chi(\boldsymbol{r}, t)$ are real fields one has $\zeta_{\boldsymbol{k}}=\zeta_{-\boldsymbol{k}}^{*}$ and $\chi_{\boldsymbol{k}}=\chi_{-\boldsymbol{k}}^{*}$. 


\subsection{Hamiltonian formulation}

It can be shown that the Föppl-von Kármán equations derives from a Hamiltonian formulation, with the general Hamiltonian of the problem yielding in dimensionless form

$$
H\left[\zeta_{\boldsymbol{k}}, p_{\boldsymbol{k}}\right]=\frac{1}{2} \int\left[p_{\boldsymbol{k}_{1}} p_{-\boldsymbol{k}_{1}}+\omega_{k}^{2} \zeta_{\boldsymbol{k}_{1}} \zeta_{-\boldsymbol{k}_{1}}\right] d^{2} \boldsymbol{k}_{1}+\frac{1}{(2 \pi)^{2}} \int T_{\boldsymbol{k}_{1} \boldsymbol{k}_{2} ; \boldsymbol{k}_{3} \boldsymbol{k}_{4}} \zeta_{\boldsymbol{k}_{1}} \zeta_{\boldsymbol{k}_{2}} \zeta_{\boldsymbol{k}_{3}} \zeta_{\boldsymbol{k}_{4}} \delta\left(\boldsymbol{k}_{1}+\boldsymbol{k}_{2}+\boldsymbol{k}_{3}+\boldsymbol{k}_{4}\right) d^{2} \boldsymbol{k}_{1234}
$$

where the momentum $p_{\boldsymbol{k}}$ is defined by the relation $p_{\boldsymbol{k}}=\dot{\zeta}_{\boldsymbol{k}}, \omega_{k}=k^{2} / 2$ and the explicit form of the tensor $T_{\boldsymbol{k}_{1} \boldsymbol{k}_{2} ; \boldsymbol{k}_{3} \boldsymbol{k}_{4}}$ will be given in eq. (26). The Hamiltonian dynamics gives the Föppl-von Kármán equations using the classical relations:

$$
\dot{\zeta}_{\boldsymbol{k}}=\frac{\delta H}{\delta p_{\boldsymbol{k}}} \quad \text { and } \quad \dot{p}_{\boldsymbol{k}}=-\frac{\delta H}{\delta \zeta_{\boldsymbol{k}}}
$$

The dynamics will be then studied using the canonical variables $A_{\boldsymbol{k}}$ corresponding to the normal modes of the linear waves, defined by:

$$
\zeta_{\boldsymbol{k}}=\frac{X_{k}}{\sqrt{2}}\left(A_{\boldsymbol{k}}+A_{-\boldsymbol{k}}^{*}\right), \quad p_{\boldsymbol{k}}=-i \frac{X_{k}^{-1}}{\sqrt{2}}\left(A_{\boldsymbol{k}}-A_{-\boldsymbol{k}}^{*}\right) \quad \text { and } \quad X_{k}=\frac{1}{\sqrt{\omega_{k}}} .
$$

Using these normal variables, the linear term in the Hamiltonian simplifies into $\omega_{k}\left|A_{\boldsymbol{k}}\right|^{2}$ which emphasizes the vibration mode at linear order.

\subsection{Kinetic equation}

Considering the different cumulants of the canonical variables, a hierarchy of equations is obtained for which an asymptotic closure can be deduced, leading to a kinetic equation that describes the slow evolution of the second order cumulant through three or four waves resonance processes depending on the geometry. Written in term of the wave number density defined as

$$
\left\langle A_{\boldsymbol{k}} A_{\boldsymbol{k}^{\prime}}^{*}\right\rangle=n(\boldsymbol{k}) \delta\left(\boldsymbol{k}-\boldsymbol{k}^{\prime}\right)
$$

and assuming moreover the isotropy of the cumulants, $n(\boldsymbol{k})=n(|\boldsymbol{k}|)=n(k)$, the kinetic equation simplifies for the elastic plates:

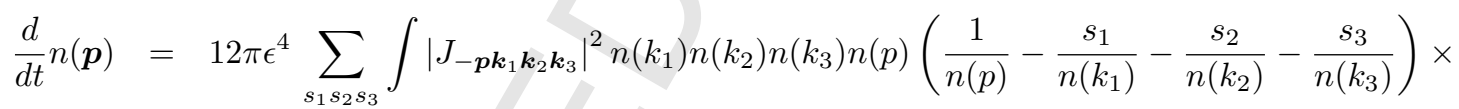

$$
\begin{aligned}
& \times \delta\left(\boldsymbol{k}_{1}+\boldsymbol{k}_{2}+\boldsymbol{k}_{3}-\boldsymbol{p}\right) \delta\left(\omega(p)-s_{1} \omega\left(k_{1}\right)-s_{2} \omega\left(k_{2}\right)-s_{3} \omega\left(k_{3}\right)\right) d \boldsymbol{k}_{123} \text {. }
\end{aligned}
$$

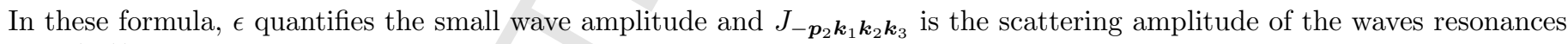
(see eq. (31)). In addition, the nonlinearity induces a frequency shift of the linear modes which reads

$$
\omega_{\boldsymbol{p}}^{\text {Ren }}=\frac{1}{2}|\boldsymbol{p}|^{2}+\epsilon^{2} \frac{3 \pi}{2}\left[\int_{0}^{p} \frac{k^{2}}{p^{2}} n(k) k d k+\int_{p}^{\infty} \frac{p^{2}}{k^{2}} n(k) k d k\right] .
$$

\subsection{Kolmogorov-Zakharov spectrum}

Beside the Rayleigh-Jeans distribution corresponding to the equipartition of the energy among the modes, additional stationary solutions of the kinetic equations can be found, corresponding to a direct cascade of energy from the large to the small scales. These so-called Kolmogorv-Zakharov spectra have a constant energy flux $P$ and exhibit different scalings depending on the plate geometry.

For elastic plate, the WTT predicts firstly KZ spectra with the same scalings than the Rayleigh-Jeans one, $n(k) \sim P^{1 / 3} k^{-2}$. It indicates a degeneracy of this solution leading to an undefined flux $P$, so that a perturbative approach has to be performed, leading to the following $\mathrm{KZ}$ spectra:

$$
n(k)=2^{4 / 3} C P^{1 / 3} \frac{\ln ^{1 / 3}\left(\kappa^{*} / k\right)}{k^{2}},
$$


where $C$ and $\kappa^{*}$ are real numbers while $P$ is the energy flux of the cascade. In the present paper we show that the interaction are local for the spectrum (22) and we obtain an upper and a lower bound for the numerical value of $C$ which reads: $0.22 \leq C \leq 0.33$. The displacement and energy spectrum can be straightforwardly deduced giving:

$$
\left\langle\left|\zeta_{k}\right|^{2}\right\rangle=2^{7 / 3} C P^{1 / 3} \frac{\ln ^{1 / 3}\left(\kappa^{*} / k\right)}{k^{4}} \text { and } E_{k}=2^{7 / 3} \pi C P^{1 / 3} k \ln ^{1 / 3}\left(\kappa^{*} / k\right)
$$

Similarly, the spectrum can be expressed in the frequency space using the relation $\omega=k^{2} / 2$, yielding:

$$
n(\omega)=C P^{1 / 3} \frac{\ln ^{1 / 3}\left(\omega^{*} / \omega\right)}{\omega} \text { and } E_{\omega}=\pi C P^{1 / 3} \ln ^{1 / 3}\left(\omega^{*} / \omega\right)
$$

Here $\omega^{*}=\kappa^{* 2} / 2$. Finally, it is interesting to write these spectra back in the physical units:

$$
n(k)=C \rho \ell^{4 / 3} P^{1 / 3} \frac{\ln ^{1 / 3}\left(k^{*} / k\right)}{k^{2}} \text { and } E_{k}=\pi C \sqrt{\frac{E}{\rho}} \ell^{7 / 3} P^{1 / 3} k \ln ^{1 / 3}\left(k^{*} / k\right),
$$

which is obviously consistent with the previous dimensional analysis $(9)$, in particular, one readily gets that the cut-off $k_{*}=\kappa^{*} / \ell$.

\section{Hamiltonian formalism.}

\subsection{Hamiltonian formalism in Fourier space.}

Despite their complexity due to highly nonlinear terms and high order derivatives, the Föppl-von Kármán equations exhibit a Hamiltonian structure. In fact, equations (1) and (2) can be deduced from a Hamilton principle in which the action $S$ defined by:

is an extreme. Here $d \boldsymbol{r}=d x d y$.

$$
S=\int\left(\frac{1}{2} \dot{\zeta}^{2}-\left[\frac{1}{8}(\Delta \zeta)^{2}-\frac{1}{2}(\Delta \chi)^{2}-\frac{1}{2} \chi\{\zeta, \zeta\}\right]\right) d \boldsymbol{r} d t
$$

The Föppl-von Kármán (1) and (2) equations are thus the Euler-Lagrange equations deduced from the action (24), naturally the Hamiltonian (6) follows directly from this variational principle.

Because the problem concerns the evolution of the wave amplitudes for different normal modes of oscillation, we will use now on variables in Fourier space. Introducing (15) and (16) into the action (24) one gets, after some algebra, the full Lagrangian density (as usual the action is $S=\int_{t_{1}}^{t_{2}} L d t$ ):

$$
L\left[\zeta_{\boldsymbol{k}}, \dot{\zeta}_{\boldsymbol{k}}\right]=\int\left[\frac{1}{2} \dot{\zeta}_{\boldsymbol{k}} \dot{\zeta}_{-\boldsymbol{k}}-\frac{1}{8}|\boldsymbol{k}|^{4} \zeta_{\boldsymbol{k}} \zeta_{-\boldsymbol{k}}\right] d^{2} \boldsymbol{k}-\frac{1}{(2 \pi)^{2}} \int T_{\boldsymbol{k}_{1} \boldsymbol{k}_{2} ; \boldsymbol{k}_{3} \boldsymbol{k}_{4}} \zeta_{\boldsymbol{k}_{1}} \zeta_{\boldsymbol{k}_{2}} \zeta_{\boldsymbol{k}_{3}} \zeta_{\boldsymbol{k}_{4}} \delta\left(\boldsymbol{k}_{1}+\boldsymbol{k}_{2}+\boldsymbol{k}_{3}+\boldsymbol{k}_{4}\right) d^{2} \boldsymbol{k}_{1234}
$$

with

$$
T_{\boldsymbol{k}_{1} \boldsymbol{k}_{2} ; \boldsymbol{k}_{3} \boldsymbol{k}_{4}}=\frac{1}{8}\left(\frac{1}{2\left|\boldsymbol{k}_{1}+\boldsymbol{k}_{2}\right|^{4}}+\frac{1}{2\left|\boldsymbol{k}_{3}+\boldsymbol{k}_{4}\right|^{4}}\right)\left(\boldsymbol{k}_{1} \times \boldsymbol{k}_{2}\right)^{2}\left(\boldsymbol{k}_{3} \times \boldsymbol{k}_{4}\right)^{2} .
$$

In (25), we have used the condensed notation $d \boldsymbol{k}_{123}=d \boldsymbol{k}_{1} d \boldsymbol{k}_{2} d \boldsymbol{k}_{3}$. The tensor $T_{12 ; 34}$ exhibits permutation symmetries $T_{12 ; 34}=$ $T_{21 ; 34}=T_{12 ; 43}=T_{34 ; 12}$ and it is a homogenous function of degree 4 in $\boldsymbol{k}$, namely:

$$
T_{\lambda \boldsymbol{k}_{1} \lambda \boldsymbol{k}_{2} ; \lambda \boldsymbol{k}_{3} \lambda \boldsymbol{k}_{4}}=\lambda^{4} T_{\boldsymbol{k}_{1} \boldsymbol{k}_{2} ; \boldsymbol{k}_{3} \boldsymbol{k}_{4}} .
$$

Finally, we emphasize that $T_{12 ; 34} \geq 0$. From the Lagrangian (25) the canonical momentum is $p_{\boldsymbol{k}}=\dot{\zeta}_{\boldsymbol{k}}$ and the Hamiltonian (17) can be deduced straightforwardly, with the followong Hamilton equations for the variables in Fourier space:

$$
\begin{aligned}
\dot{\zeta}_{\boldsymbol{k}} & =p_{\boldsymbol{k}} \\
\dot{p}_{\boldsymbol{k}} & =-\frac{1}{4} \boldsymbol{k}^{4} \zeta_{\boldsymbol{k}}-\frac{4}{(2 \pi)^{2}} \int T_{-\boldsymbol{k} \boldsymbol{k}_{2} ; \boldsymbol{k}_{3} \boldsymbol{k}_{4}} \zeta_{\boldsymbol{k}_{2}} \zeta_{\boldsymbol{k}_{3}} \zeta_{\boldsymbol{k}_{4}} \delta\left(\boldsymbol{k}-\boldsymbol{k}_{2}-\boldsymbol{k}_{3}-\boldsymbol{k}_{4}\right) d^{2} \boldsymbol{k}_{234}
\end{aligned}
$$

where the cubic nonlinearity of the interaction is clearly apparent in the second equation. 


\subsection{Dynamics equations in normal variables}

Introducing the transformations (18) into the general Hamiltonian (17) one finds that $H$ becomes the usual Hamiltonian of weakly interacting oscillators with associated frequency $\omega_{\boldsymbol{k}}$ (obeying the dispersion relation eq. 14):

$$
H=\int \omega_{\boldsymbol{k}} A_{\boldsymbol{k}} A_{\boldsymbol{k}}^{*} d \boldsymbol{k}+\frac{1}{4(2 \pi)^{2}} \int X_{\boldsymbol{k}_{1}} X_{\boldsymbol{k}_{2}} X_{\boldsymbol{k}_{3}} X_{\boldsymbol{k}_{4}} T_{\boldsymbol{k}_{1} \boldsymbol{k}_{2} ; \boldsymbol{k}_{3} \boldsymbol{k}_{4}} \sum_{s_{1} s_{2} s_{3} s_{4}} A_{\boldsymbol{k}_{1}}^{s_{1}} A_{\boldsymbol{k}_{2}}^{s_{2}} A_{\boldsymbol{k}_{3}}^{s_{3}} A_{\boldsymbol{k}_{4}}^{s_{4}} \delta\left(\boldsymbol{k}_{1}+\boldsymbol{k}_{2}+\boldsymbol{k}_{3}+\boldsymbol{k}_{4}\right) d \boldsymbol{k}_{1234} \text {. }
$$

We shall use along the paper the notation introduced by Newell et al. [4] : $A_{k}^{s}$ with $s=+,-$, witnessing the two directions of propagation for each mode. It means that $A_{k}^{+}=A_{\boldsymbol{k}}$ and $A_{\boldsymbol{k}}^{-}=A_{-\boldsymbol{k}}^{*}$ then $A_{-\boldsymbol{k}}^{-s}=A_{k}^{s *}$ and the sum is over the values $s_{i}=-1$ and +1 .

Weak turbulence is valid for small amplitudes, that is the local slope of the deformation is the small parameter $\epsilon$. The order of magnitude of the deformation is also $\epsilon$, following $\zeta_{\boldsymbol{k}} \simeq O(\epsilon)$. Rescaling $\zeta_{\boldsymbol{k}} \longrightarrow \epsilon \zeta_{\boldsymbol{k}}$ so that $A_{\boldsymbol{k}} \longrightarrow \epsilon A_{\boldsymbol{k}}$, one concludes that the rescaled Hamiltonian is of the form

$$
H=\epsilon^{2} H_{2}+\epsilon^{4} H_{4} \text {. }
$$

Up to the lowest order (order $\epsilon^{2}$ ) the Hamiltonian is simply $H \approx \epsilon^{2} H_{2}=\epsilon^{2} \int \omega_{\boldsymbol{k}} A_{\boldsymbol{k}} A_{\boldsymbol{k}}^{*} d \boldsymbol{k}$, the sum of the normal modes of oscillation. The wave interactions appear at the fourth order, with the term $\epsilon^{4} H_{4}$.

From the Hamiltonian we obtain the following equations of the dynamics for the normal variables:

$$
\frac{d A_{\boldsymbol{k}}^{s}}{d t}+i s \omega_{s \boldsymbol{k}} A_{\boldsymbol{k}}^{s}=\epsilon^{2} \sum_{s_{1} s_{2} s_{3}} \int L_{\boldsymbol{k k}_{1} \boldsymbol{k}_{2} \boldsymbol{k}_{3}}^{s s_{1} s_{2} s_{3}} A_{\boldsymbol{k}_{1}}^{s_{1}} A_{\boldsymbol{k}_{2}}^{s_{2}} A_{\boldsymbol{k}_{3}}^{s_{3}} \delta\left(\boldsymbol{k}_{1}+\boldsymbol{k}_{2}+\boldsymbol{k}_{3}-\boldsymbol{k}\right) d \boldsymbol{k}_{123},
$$

where we have used the symmetries of the tensor $T_{\boldsymbol{k}_{1} \boldsymbol{k}_{2} ; \boldsymbol{k}_{3} \boldsymbol{k}_{4}}$ and the nonlinear interaction coefficient reads ${ }^{1}$

$$
L_{\boldsymbol{k} \boldsymbol{k}_{1} \boldsymbol{k}_{2} \boldsymbol{k}_{3}}^{s s_{1} s_{2} s_{3}}=-\frac{i s}{(2 \pi)^{2}} J_{-\boldsymbol{k} \boldsymbol{k}_{1} \boldsymbol{k}_{2} \boldsymbol{k}_{3}}
$$

Here, we have defined for convenience

$$
J_{\boldsymbol{k}_{1} \boldsymbol{k}_{2} \boldsymbol{k}_{3} \boldsymbol{k}_{4}}=\frac{1}{3} X_{\boldsymbol{k}_{1}} X_{\boldsymbol{k}_{2}} X_{\boldsymbol{k}_{3}} X_{\boldsymbol{k}_{4}}\left(T_{\boldsymbol{k}_{1} \boldsymbol{k}_{2} ; \boldsymbol{k}_{3} \boldsymbol{k}_{4}}+T_{\boldsymbol{k}_{1} \boldsymbol{k}_{3} ; \boldsymbol{k}_{2} \boldsymbol{k}_{4}}+T_{\boldsymbol{k}_{1} \boldsymbol{k}_{4} ; \boldsymbol{k}_{3} \boldsymbol{k}_{2}}\right),
$$

which is the relevant scattering amplitude coefficient that will appear in the final kinetic equation.

In the weak turbulence theory the $L$ functions satisfy usually a number of general properties [4]. In our particular case it is easy to see that the following properties are satisfied :

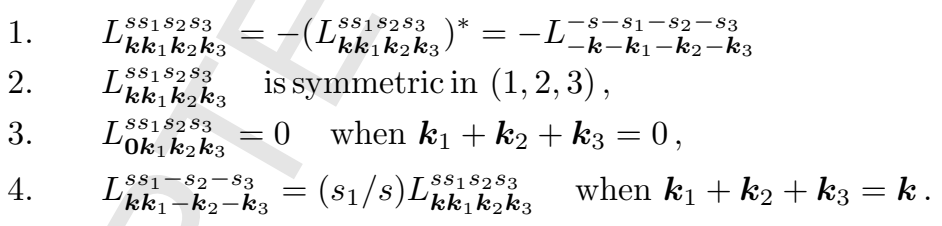

\section{Kinetic equation and statistical descripition}

\subsection{Moments and Cumulants}

Up to now the infinite set of dynamical equations (29) describe the motion of the plate, in the frame of the Föppl-von Kármán equations, without any approximation. Nevertheless, from now on, because of the random aspects of the fluctuations due to the high numbers of oscillating modes, we shall treat the system statistically. To perform this analysis, we consider the behavior of the moments and cumulants in both the physical (or real) and the Fourier spaces.

Although the physical variables in the problem are the canonical fields $\zeta(\boldsymbol{r}, t)$ and $p(\boldsymbol{r}, t)$, for the following it will be more adequate to define the "normal" field $u^{s}(\boldsymbol{r}, t)$, which is the Fourier transform of the normal mode of oscillation $A_{\boldsymbol{k}}^{s}$, namely:

$$
u^{s}(\boldsymbol{r}, t) \equiv \frac{1}{2 \pi} \int A_{\boldsymbol{k}}^{s} e^{i \boldsymbol{k} \cdot \boldsymbol{r}} d \boldsymbol{k} .
$$

\footnotetext{
${ }^{1}$ It is convenient to keep the indices $s_{i}$ only for the sake of generality.
} 
Because of the linear relationship between $A_{\boldsymbol{k}}^{s}$ and, $\zeta_{\boldsymbol{k}}$ and $p_{\boldsymbol{k}}$, one expects that $u^{s}(\boldsymbol{r}, t)$ is a linear (but non local) functional of the fields $\zeta(\boldsymbol{r}, t)$ and $p(\boldsymbol{r}, t)$. If the statistic of the fields $\zeta(\boldsymbol{r}, t)$ and $p(\boldsymbol{r}, t)$ are homogeneous, then the statistic of the two fields $u^{s}(\boldsymbol{r}, t)$ is also homogeneous.

The statistical properties of these fields depend on a joint probability distribution function $(\mathrm{PDF})$ at all points $\mathcal{P}[u(\cdot)]$, whose existence is assumed, even though we cannot compute it. Over the last 50 years, two different approaches have been developed, leading in practice to the same final results. The first one is based on the random phase approximation (RPA) for the Fourier modes[1,3], while the second one is based on the cumulant expansion of the statistical properties of the spatial homogeneous fields [2].

The method of random phase approximation (RPA) which has been extensibly used [1,3] in the theory of wave turbulence, is based on the hypothesis that all $n$-modes PDF in Fourier space (directly related to the $n$-point PDF $P^{n}$ in real space) are a product of 1 -mode PDFs, and that the phases take values uniformly distributed on $[0,2 \pi]$. However, this approximation has been proven to be right only recently by Choi et al. [44]. Indeed, if we assume that the initial data satisfies the random phase approximation properties described above, then, this property holds over the nonlinear time. Choi et al. derived an evolution equation for the full PDF and have shown that under weak wave interaction the RPA formulation is sufficient for the wave turbulence closure. Despite the fact that the RPA approach to the problem of weakly interacting waves may drive to better developments for future theories on non-Gaussianity of wave turbulence and intermittency (due to the knowledge of the full PDF), we develop in the present paper the second method, introduced by Benney and Saffman 50 years ago [2], that gives, in our opinion, a more elegant asymptotic closure.

This method, known as the cumulant expansion, has the advantage to not impose any condition to the PDF, with the exception that the statistics of the system for infinitely separated points are uncorrelated at some initial time. We consider thus an infinite system and make the assumption of spatial homogeneity. It means that the moments defined above are invariant under spatial translation, so that they depend only on the relative geometry. This allows us to have a better physical intuition about what we are averaging over. More precisely, because of the spatial homogeneity we can define the moment of order $n$ as

$$
\left\langle u^{s}(\boldsymbol{x}) u^{s_{1}}\left(\boldsymbol{x}+\boldsymbol{r}_{1}\right) \ldots u^{s_{n-1}}\left(\boldsymbol{x}+\boldsymbol{r}_{n-1}\right)\right\rangle=\frac{1}{V} \int u^{s}(\boldsymbol{x}) u^{s_{1}}\left(\boldsymbol{x}+\boldsymbol{r}_{1}\right) \ldots u^{s_{n-1}}\left(\boldsymbol{x}+\boldsymbol{r}_{n-1}\right) d \boldsymbol{x}
$$

where the integral is over all the volume $V$ of the system.

Unfortunately, for the following the moments are not adequate, because when the distances $\left|\boldsymbol{r}_{1}\right|,\left|\boldsymbol{r}_{2}\right|, \ldots,\left|\boldsymbol{r}_{n-1}\right|$ tend independently to infinity, the moments do not decay to zero in the physical space. Therefore, the Fourier transforms of the moments are not ordinary functions, but generalized functions that contains Dirac $\delta$-functions, and as we shall see later, it is necessarily to have a good tracking of the Dirac- $\delta$ singularities. Therefore, the use of moments would complicate in excess this procedure. For this reason we shall use cumulants, which are directly related to the moments by a one to one relation. The cumulants Fourier transforms are ordinary functions if the statistics of the system, for infinitely separated points, is uncorrelated (See the Appendix 10.1 for details on the Moment-Cumulant relationship).

For the first two order cumulants, their relations with the moments read

$$
\begin{aligned}
R^{(1) s}(t) & =\left\{u^{s}(\boldsymbol{x})\right\}=\left\langle u^{s}(\boldsymbol{x})\right\rangle, \\
R^{(2) s s_{1}}(t) & =\left\{u^{s}(\boldsymbol{x}) u^{s_{1}}\left(\boldsymbol{x}+\boldsymbol{r}_{1}\right)\right\}=\left\langle u^{s}(\boldsymbol{x}) u^{s_{1}}\left(\boldsymbol{x}+\boldsymbol{r}_{1}\right)\right\rangle-\left\langle u^{s}(\boldsymbol{x})\right\rangle\left\langle u^{s}(\boldsymbol{x})\right\rangle,
\end{aligned}
$$

and highest order cumulants can be similarly deduced (see Appendix 10.1).

Let be $R^{(n) s s_{1} \cdots s_{n-1}}\left(\boldsymbol{r}_{1}, \ldots, \boldsymbol{r}_{n-1}\right)$, the $n$-th order cumulant, then, we write the Fourier transform of $Q^{(n)}$ of the $n$-th order cumulant as

$$
Q^{(n) s s_{1} \cdots s_{n-1}}\left(\boldsymbol{k}_{1}, \ldots, \boldsymbol{k}_{(n-1)}\right)=\frac{1}{(2 \pi)^{n-1}} \int R^{(n) s s_{1} \cdots s_{n-1}}\left(\boldsymbol{r}_{1}, \ldots, \boldsymbol{r}_{n-1}\right) e^{-i \boldsymbol{k}_{1} \cdot \boldsymbol{r}_{1} \cdots-i \boldsymbol{k}_{n-1} \cdot \boldsymbol{r}_{n-1}} d \boldsymbol{r}_{1} \ldots d \boldsymbol{r}_{n-1}
$$

The $n$-th order cumulant, $Q^{(n)}$, is a smooth function in the Fourier space, while the cumulant in the real space goes to zero for two infinitely separated points. This assumption is in fact only necessary for the initial state (See Section 4.3). Obviously, we can easily recover the moments knowing the cumulants in Fourier space. From the definition (33) one has

$$
\left\langle A_{\boldsymbol{k}}^{s} A_{\boldsymbol{k}_{1}}^{s_{1}} \ldots A_{\boldsymbol{k}_{n-1}}^{s_{n-1}}\right\rangle=\frac{1}{(2 \pi)^{n}} \int\left\langle u^{s}(\boldsymbol{x}) u^{s_{1}}\left(\boldsymbol{x}+\boldsymbol{r}_{1}\right) \ldots u^{s_{n-1}}\left(\boldsymbol{x}+\boldsymbol{r}_{n-1}\right)\right\rangle e^{-i \boldsymbol{k} \cdot \boldsymbol{x}} e^{-i \boldsymbol{k}_{1} \cdot\left(\boldsymbol{x}+\boldsymbol{r}_{1}\right)} \ldots e^{-i \boldsymbol{k}_{n-1} \cdot\left(\boldsymbol{x}+\boldsymbol{r}_{n-1}\right)} d \boldsymbol{x} d \boldsymbol{r}_{1} \ldots d \boldsymbol{r}_{n-1}
$$


Then replacing the relations obtained in (34), and using (35), the first order moment and cumulant are related by:

$$
\left\langle A_{\boldsymbol{p}_{1}}^{l_{1}}\right\rangle=\int R^{(1) l_{1}} e^{-i \boldsymbol{p}_{1} \cdot \boldsymbol{x}} d \boldsymbol{x}=(2 \pi) Q^{(1) l_{1}} \delta\left(\boldsymbol{p}_{1}\right)
$$

As already said, the plate preserves the center of mass momentum, and we shall assume it is at rest in the reference frame. Then, it is natural to assume that the mean values of the displacement and the momentum and consequently the mean value of the field $u^{s}(\boldsymbol{x})$ are null. Therefore, it is expected that $Q^{(1) l_{1}}=0$. This condition may not be true in some situations as in the case of wave condensation of classical nonlinear waves [45]. However, in the present case, it is always zero, so that from now on we take $Q^{(1) l_{1}}=0$. Under this assumption, a larger number of simplifications are obtained. Firstly, the second order moment of the Fourier amplitudes is simply related to the second order cumulant by

$$
\left\langle A_{\boldsymbol{p}_{1}}^{l_{1}} A_{\boldsymbol{p}_{2}}^{l_{2}}\right\rangle=\int R^{(2) l_{1} l_{2}}(\boldsymbol{r}) e^{-i \boldsymbol{p}_{2} \cdot \boldsymbol{r}} d \boldsymbol{r} \delta\left(\boldsymbol{p}_{1}+\boldsymbol{p}_{2}\right)=(2 \pi) Q^{(2) l_{1} l_{2}}\left(\boldsymbol{p}_{2}\right) \delta\left(\boldsymbol{p}_{1}+\boldsymbol{p}_{2}\right) .
$$

Notice that $Q^{(2) l_{1} l_{2}}\left(\boldsymbol{p}_{2}\right)=Q^{(2) l_{2} l_{1}}\left(-\boldsymbol{p}_{2}\right)$ and that the wave number density satisfies:

$$
n(l p)=2 \pi Q^{(2)-l l}(\boldsymbol{p}) .
$$

Similarly, a one to one relation between the higher order moments and cumulants can be derived in the Fourier space (see Appendix 10.1).

\subsection{The moments and cumulant hierarchy}

From (29) a hierarchy of equations for the evolution of the moments $\left\langle A_{\boldsymbol{p}_{1}}^{l_{1}} A_{\boldsymbol{p}_{2}}^{l_{2}} \ldots A_{\boldsymbol{p}_{n}}^{l_{n}}\right\rangle$ in Fourier space can be written :

$$
\begin{aligned}
\frac{d}{d t}\left\langle A_{\boldsymbol{p}_{1}}^{l_{1}} A_{\boldsymbol{p}_{2}}^{l_{2}} \ldots A_{\boldsymbol{p}_{n}}^{l_{n}}\right\rangle= & \mathcal{P}_{1^{\prime} \ldots n^{\prime}}\left\langle\frac{d A_{\boldsymbol{p}_{1}}^{l_{1}}}{d t} A_{\boldsymbol{p}_{2}}^{l_{2}} \ldots A_{\boldsymbol{p}_{n}}^{l_{n}}\right\rangle= \\
= & \mathcal{P}_{1^{\prime} \ldots n^{\prime}}\left(-i l_{1} \omega\left(\boldsymbol{p}_{1}\right)\left\langle A_{\boldsymbol{p}_{1}}^{l_{1}} A_{\boldsymbol{p}_{2}}^{l_{2}} \ldots A_{\boldsymbol{p}_{n}}^{l_{n}}\right\rangle\right)+ \\
& \epsilon^{2} \mathcal{P}_{1^{\prime} \ldots n^{\prime}} \sum_{s_{1} s_{2} s_{3}} \int L_{\boldsymbol{p}_{n} \boldsymbol{k}_{1} \boldsymbol{k}_{2} \boldsymbol{k}_{3}}^{l_{n} s_{1} s_{2} s_{3}}\left\langle A_{\boldsymbol{p}_{1}}^{l_{1}} \ldots A_{\boldsymbol{p}_{n-1}}^{l_{n-1}} A_{\boldsymbol{k}_{1}}^{s_{1}} A_{\boldsymbol{k}_{2}}^{s_{2}} A_{\boldsymbol{k}_{3}}^{s_{3}}\right\rangle \delta\left(\boldsymbol{k}_{1}+\boldsymbol{k}_{2}+\boldsymbol{k}_{3}-\boldsymbol{p}_{n}\right) d \boldsymbol{k}_{123} .
\end{aligned}
$$

The resulting dynamics of the $n$-th order moments depends explicitly upon the $(n+2)$-th order ones. This hierarchy is linear in the moments $\left\langle A_{\boldsymbol{p}_{1}}^{l_{1}} A_{\boldsymbol{p}_{2}}^{l_{2}} \ldots A_{\boldsymbol{p}_{n}}^{l_{n}}\right\rangle$ like the BBGKY hierarchy for the kinetic theory of gases. From now on, we shall use the notation where $\mathcal{P}_{i^{\prime}}$ represents the permutation over the indices $l_{i}$ and the wave numbers $\boldsymbol{p}_{i}$. Later on, we shall also use the permutation $\mathcal{P}_{i}$ that runs over the indices $s_{i}$ and wave numbers $\boldsymbol{k}_{i}$.

In particular from the equation (39) we can build a non linear cumulant hierarchy using the relations (38) and (120) derived in Appendix 10.1. After some algebra one gets for $n=2$

$$
\begin{aligned}
\frac{d}{d t} Q^{(2) l_{1} l_{2}}\left(\boldsymbol{p}_{2}\right) & =\left(-i l_{1} \omega\left(\boldsymbol{p}_{1}\right)-i l_{2} \omega\left(\boldsymbol{p}_{2}\right)\right) Q^{(2) l_{1} l_{2}}\left(\boldsymbol{p}_{2}\right)+ \\
& +\epsilon^{2} \mathcal{P}_{1^{\prime} 2^{\prime}} \sum_{s_{1} s_{2} s_{3}} \int L_{\boldsymbol{p}_{2} \boldsymbol{k}_{1} \boldsymbol{k}_{2} \boldsymbol{k}_{3}}^{l_{2} s_{1} s_{2} s_{3}} Q^{(4) l_{1} s_{1} s_{2} s_{3}}\left(\boldsymbol{k}_{1}, \boldsymbol{k}_{2}, \boldsymbol{k}_{3}\right) \delta\left(\boldsymbol{p}_{1}+\boldsymbol{k}_{1}+\boldsymbol{k}_{2}+\boldsymbol{k}_{3}\right) d \boldsymbol{k}_{123}+ \\
& +\epsilon^{2}(2 \pi) \mathcal{P}_{1^{\prime} 2^{\prime}} \sum_{s_{1} s_{2} s_{3}} \int L_{\boldsymbol{p}_{2} \boldsymbol{k}_{1} \boldsymbol{k}_{2} \boldsymbol{k}_{3}}^{l_{2} s_{1} s_{2} s_{3}} \mathcal{P}_{123} Q^{(2) l_{1} s_{1}}\left(\boldsymbol{k}_{1}\right) Q^{(2) s_{2} s_{3}}\left(\boldsymbol{k}_{3}\right) \delta\left(\boldsymbol{p}_{1}+\boldsymbol{k}_{1}\right) \delta\left(\boldsymbol{k}_{2}+\boldsymbol{k}_{3}\right) d \boldsymbol{k}_{123},
\end{aligned}
$$

where $\boldsymbol{p}_{1}+\boldsymbol{p}_{2}=0$. Other cumulant equations are explicitly given in the Appendix 10.2.

Up to now we have an exact, but infinite, hierarchy of equations for the cumulants $Q^{(n)}$. The next step consists in finding a closure that allows us to obtain a consistent and closed set of equations. This is possible because of the dispersive nature of the waves that creates a fast decorrelation between the different modes, and because of the existence of a scale separation between the linear time $\omega(\boldsymbol{p})^{-1}$ and the nonlinear interaction time scales characterized by the small nonlinear coupling term leading to a resonant interaction process. This latter process regenerates the higher-order cumulants in a special way by producing long time cumulative effects. 
More precisely, if $\epsilon^{2}$ is the small parameter, defined by the ratio between the linear and the nonlinear terms, then the nonlinear time scale is of order $\epsilon^{-4}$ compared to the linear time scale of order one. This property will be exploited from a mathematical point of view, using a multiple time scale perturbation scheme, as exposed for the three waves kinetic equation by Newell et al. [4]. The general 4-waves interaction, that is also operating here, has been originally derived, using a slightly different method, by Newell in 1968 [46], but in a different context.

\subsection{Asymptotic perturbation expansion for the cumulants hierarchy}

The closure of this infinite hierarchy of equations for the cumulants is thus obtained by a perturbative method. That is by looking to the solutions of the cumulant hierarchy as asymptotic expansions ${ }^{2}$ in power of the small parameter $\epsilon$ and by solving this hierarchy order by order. Let us consider the formal expansion ${ }^{3}$

$$
Q^{(n) l_{1} \ldots l_{n}}\left(\boldsymbol{p}_{2}, \ldots, \boldsymbol{p}_{n}\right)=Q_{0}^{(n) l_{1} \ldots l_{n}}\left(\boldsymbol{p}_{2}, \ldots, \boldsymbol{p}_{n}\right)+\epsilon^{2} Q_{2}^{(n) l_{1} \ldots l_{n}}\left(\boldsymbol{p}_{2}, \ldots, \boldsymbol{p}_{n}\right)+\epsilon^{4} Q_{4}^{(n) l_{1} \ldots l_{n}}\left(\boldsymbol{p}_{2}, \ldots, \boldsymbol{p}_{n}\right)+\cdots
$$

Up to order $\epsilon^{0}$ one gets that any $n$-th order cumulants $Q^{(n)}$ satisfy the following ordinary differential equations:

$$
\frac{d}{d t} Q_{0}^{(n) l_{1} \ldots l_{n}}\left(\boldsymbol{p}_{2}, \ldots, \boldsymbol{p}_{n}\right)=-\left(i l_{1} \omega\left(\boldsymbol{p}_{1}\right)+\cdots+i l_{n} \omega\left(\boldsymbol{p}_{n}\right)\right) Q_{0}^{(n) l_{1} \ldots l_{n}}\left(\boldsymbol{p}_{2}, \ldots, \boldsymbol{p}_{n}\right)
$$

with $\boldsymbol{p}_{1}+\cdots+\boldsymbol{p}_{n}=0$. After an integration of this linear oscillator in time one retrieves a pure linear oscillation in time,

$$
Q_{0}^{(n) l_{1} \ldots l_{n}}\left(\boldsymbol{p}_{2}, \ldots, \boldsymbol{p}_{n}\right)=q_{0}^{(n) l_{1} \ldots l_{n}}\left(\boldsymbol{p}_{2}, \ldots, \boldsymbol{p}_{n}\right) e^{-i\left(l_{1} \omega\left(\boldsymbol{p}_{1}\right)+\cdots+l_{n} \omega\left(\boldsymbol{p}_{n}\right)\right) t}
$$

with the functions $q_{0}^{(n) l_{1} \ldots l_{n}}\left(\boldsymbol{p}_{2}, \ldots, \boldsymbol{p}_{n}\right)$ being constant at this order of the expansion. In fact, as we will demonstrate below, this solution is valid only for times scales such that $t \ll \epsilon^{-2}$ since the next order solution in the regular expansion exhibits a secular term that grows linearly with $t$, becoming dominant in the long time limit. To remove these secular terms, which are responsible for the slow spectral energy transfer between modes, we develop a multi-scale perturbation expansion in time. In this scheme, one assumes a slow dynamic evolution for $q_{0}^{(n) l_{1} \ldots l_{n}}\left(\boldsymbol{p}_{2}, \ldots, \boldsymbol{p}_{n}, t\right)$, that can be expressed as a formal asymptotic series for its time derivative, yielding :

$$
\frac{d}{d t} q_{0}^{(n) l_{1} \ldots l_{n}}\left(\boldsymbol{p}_{2}, \ldots, \boldsymbol{p}_{n}\right)=\epsilon^{2} F_{2}^{(n) l_{1} \ldots l_{n}}\left(\boldsymbol{p}_{2}, \ldots, \boldsymbol{p}_{n}\right)+\epsilon^{4} F_{4}^{(n) l_{1} \ldots l_{n}}\left(\boldsymbol{p}_{2}, \ldots, \boldsymbol{p}_{n}\right)+\ldots
$$

The oscillatory nature of the system suggests the following change of variable for all cumulants and for all orders in the cumulant expansion :

$$
Q_{s}^{(n) l_{1} \ldots l_{n}}\left(\boldsymbol{p}_{2}, \ldots, \boldsymbol{p}_{n}\right)=q_{s}^{(n) l_{1} \ldots l_{n}}\left(\boldsymbol{p}_{2}, \ldots, \boldsymbol{p}_{n}, t\right) e^{-i\left(l_{1} \omega\left(\boldsymbol{p}_{1}\right)+\cdots+l_{n} \omega\left(\boldsymbol{p}_{n}\right)\right) t}
$$

where the higher order perturbation terms $q_{s}^{(n) l_{1} \ldots l_{n}}\left(\boldsymbol{p}_{2}, \ldots, \boldsymbol{p}_{n}, t\right)$ might depend on the fast time scale only for $s>0$. Writing equation (40) for the new variables (43) up to order $\epsilon^{2}$ one gets

$$
\begin{aligned}
\frac{d}{d t} q_{2}^{(2) l_{1} l_{2}}\left(\boldsymbol{p}_{2}\right) & =-F_{2}^{(2) l_{1} l_{2}}\left(\boldsymbol{p}_{2}\right)+ \\
& +\mathcal{P}_{1^{\prime} 2^{\prime}} \sum_{s_{1} s_{2} s_{3}} \int L_{\boldsymbol{p}_{2} \boldsymbol{k}_{1} \boldsymbol{k}_{2} \boldsymbol{k}_{3}}^{l_{2} s_{1} s_{2} s_{3}} q_{0}^{(4) l_{1} s_{1} s_{2} s_{3}}\left(\boldsymbol{k}_{1}, \boldsymbol{k}_{2}, \boldsymbol{k}_{3}\right) e^{-i t \Omega_{p_{2} k_{1} k_{2} k_{3}}^{-l_{2} s_{1} s_{2} s_{3}} \delta\left(\boldsymbol{p}_{1}+\boldsymbol{k}_{1}+\boldsymbol{k}_{2}+\boldsymbol{k}_{3}\right) d \boldsymbol{k}_{123}+} \\
& +(2 \pi) \mathcal{P}_{1^{\prime} 2^{\prime}} \sum_{s_{1} s_{2} s_{3}} \int L_{\boldsymbol{p}_{2} \boldsymbol{k}_{1} \boldsymbol{k}_{2} \boldsymbol{k}_{3}}^{l_{2} s_{1} s_{2} s_{3}} \mathcal{P}_{123} q_{0}^{(2) l_{1} s_{1}}\left(\boldsymbol{k}_{1}\right) q_{0}^{(2) s_{2} s_{3}}\left(\boldsymbol{k}_{3}\right) e^{-i t \Omega_{p_{2} k_{1} k_{2} k_{3}}^{-l_{2} s_{1} s_{3}} \delta\left(\boldsymbol{p}_{1}+\boldsymbol{k}_{1}\right) \delta\left(\boldsymbol{k}_{2}+\boldsymbol{k}_{3}\right) d \boldsymbol{k}_{123}},
\end{aligned}
$$

where $\boldsymbol{p}_{1}+\boldsymbol{p}_{2}=0$ and we have used the short notation $\Omega_{p_{2} k_{1} k_{2} k_{3}}^{l_{2} s_{1} s_{2} s_{3}}=l_{2} \omega\left(\boldsymbol{p}_{2}\right)+s_{1} \omega\left(\boldsymbol{k}_{1}\right)+s_{2} \omega\left(\boldsymbol{k}_{2}\right)+s_{3} \omega\left(\boldsymbol{k}_{3}\right)$. The time dependance on (44) is only given by $e^{-i t \Omega_{p_{2} k_{1} k_{2} k_{3}}^{-l_{2} s_{1} s_{2} s_{3}}}$, hence can be easily integrated in time to obtain $q_{2}^{(2) l_{1} l_{2}}\left(\boldsymbol{p}_{2}, t\right)$. Expanding the sums and

\footnotetext{
${ }^{2}$ Although, it is not necessarily a convergent series, we do ask here that the sequence is well ordered.

${ }^{3}$ As we shall see later, higher order cumulants $(n>2)$ will develop, in general, non-smooth terms at long time. Therefore it will be convenient to have in mind that the formal asymptotic expansion is done in the physical space, where the formal series expansion reads $R^{(n)}=R_{0}^{(n)}+\epsilon^{2} R_{2}^{(n)}+\epsilon^{4} R_{4}^{(n)}+\cdots$
} 
permutations on the right hand side term of (44) one observes that for some terms $\Omega_{p_{2} k_{1} k_{2} k_{3}}^{-l_{2} s_{1} s_{2} s_{3}}$ vanish identically leading to resonant terms which grows linearly in time. Therefore, we can rewrite equation (44) as

$q_{2}^{(2) l_{1} l_{2}}\left(\boldsymbol{p}_{2}, t\right)=\alpha^{(2) l_{1} l_{2}}\left(\boldsymbol{p}_{2}\right)-t\left(F_{2}^{(2) l_{1} l_{2}}\left(\boldsymbol{p}_{2}\right)-3 \mathcal{P}_{1^{\prime} 2^{\prime}}\left[\sum_{s} 2 \pi \int L_{\boldsymbol{p}_{2} \boldsymbol{p}_{2}-\boldsymbol{k} \boldsymbol{k}}^{l_{2} l_{2}-s s} q_{0}^{(2)-s s}(\boldsymbol{k}) d \boldsymbol{k}\right] q_{0}^{(2) l_{1} l_{2}}\left(\boldsymbol{p}_{2}\right)\right)+$ "non resonant terms",

where $\alpha^{(2) l_{1} l_{2}}\left(\boldsymbol{p}_{2}\right)$ is the integration constant (see Appendix 10.3 for the full expression (123) and more details on the multiscale perturbation). Since we are interested in solutions of $q_{2}^{(2) l_{1} l_{2}}\left(\boldsymbol{p}_{2}, t\right)$ that remains bounded for times of order $O\left(\epsilon^{-2}\right)$ we remove the resonant term setting the slow dynamic evolution of $q_{0}^{(2) l_{1} l_{2}}\left(\boldsymbol{p}_{2}\right)$ at times scales $O\left(\epsilon^{-2}\right)$ by

$$
F_{2}^{(2) l_{1} l_{2}}\left(\boldsymbol{p}_{2}\right)=3 \mathcal{P}_{1^{\prime} 2^{\prime}}\left[\sum_{s} 2 \pi \int L_{\boldsymbol{p}_{2} \boldsymbol{p}_{2}-\boldsymbol{k} \boldsymbol{k}}^{l_{2} l_{2}-s s} q_{0}^{(2)-s s}(\boldsymbol{k}) d \boldsymbol{k}\right] q_{0}^{(2) l_{1} l_{2}}\left(\boldsymbol{p}_{2}\right) .
$$

This corresponds to a nonlinear shift to the natural dispersion relation for the frequency known as a frequency renormalization which is reduced up to the end to the simplified expression (21). Similarly, it is possible to show that for all cumulant the resonant terms at order $\epsilon^{2}$ provides the same frequency shift (see Appendix 10.3 for more details).

$$
F_{2}^{(n) l_{1} \ldots l_{n}}\left(\boldsymbol{p}_{2}, \ldots, \boldsymbol{p}_{n}\right)=3 \mathcal{P}_{1^{\prime} \ldots n^{\prime}}\left[\sum_{s} \int L_{\boldsymbol{p}_{n} \boldsymbol{p}_{n}-\boldsymbol{k} \boldsymbol{k}}^{l_{n} l_{n}-s s}(2 \pi) q_{0}^{(2)-s s}(\boldsymbol{k}) d \boldsymbol{k}\right] q_{0}^{(n) l_{1} \ldots l_{n}}\left(\boldsymbol{p}_{2}, \ldots, \boldsymbol{p}_{n}\right) .
$$

Remarkably, at this order $\epsilon^{2}$ in the expansion, the off-diagonal second order cumulant $q_{0}^{(2)-l l}(\boldsymbol{p})$ does not evolve since $\frac{d}{d t} q_{0}^{(2)-l l}(\boldsymbol{p})=\epsilon^{2} F_{2}^{(2)-l l}(\boldsymbol{p})+\mathcal{O}\left(\epsilon^{4}\right)$ with $F_{2}^{(2)-l l}(\boldsymbol{p})=0$ and one needs in fact to go up to the next order in the expansion to capture the slow dynamics of this cumulant ${ }^{4}$ A straightforward, but cumbersome, calculation at order $\epsilon^{4}$ following the same multiscale perturbation scheme leads to a close equation for the second order cumulant (see Appendix 10.4). The final result gives different informations depending on the cases $l_{1}=l_{2}$ or $l_{1}=-l_{2}$. For $l_{1}=l_{2}$, one has that the slow dynamical evolution of the diagonal element of the second order cumulant at order $\epsilon^{4}$ is

$$
F_{4}^{(2) l_{2} l_{2}}\left(\boldsymbol{p}_{2}\right)=i \epsilon^{4}\left(\omega_{4}^{l_{2}}\left(\boldsymbol{p}_{2}\right)+\omega_{4}^{l_{2}}\left(-\boldsymbol{p}_{2}\right)\right) q_{0}^{(2) l_{2} l_{2}}\left(\boldsymbol{p}_{2}\right)-\epsilon^{4}\left(\Gamma^{l_{2}}\left(\boldsymbol{p}_{2}\right)+\Gamma^{l_{2}}\left(-\boldsymbol{p}_{2}\right)\right) q_{0}^{(2) l_{2} l_{2}}\left(\boldsymbol{p}_{2}\right)
$$

where

$$
\omega_{4}^{l_{2}}\left(\boldsymbol{p}_{2}\right)=6(2 \pi)^{2} \sum_{s_{1} s_{2} s_{3}} P \int \frac{\left|L_{\boldsymbol{p}_{2} \boldsymbol{k}_{1} \boldsymbol{k}_{2} \boldsymbol{k}_{3}}^{l_{2} s_{1} s_{2} s_{3}}\right|^{2}}{\Omega_{\boldsymbol{p}_{2} \boldsymbol{k}_{1} \boldsymbol{k}_{2} \boldsymbol{k}_{3}}^{-l_{2} s_{2} s_{3}}} \mathcal{P}_{123}\left(\frac{s_{3}}{l_{2}}\right) q_{0}^{(2)-s_{1} s_{1}}\left(\boldsymbol{k}_{1}\right) q_{0}^{(2)-s_{2} s_{2}}\left(\boldsymbol{k}_{2}\right) \delta\left(\boldsymbol{k}_{1}+\boldsymbol{k}_{2}+\boldsymbol{k}_{3}-\boldsymbol{p}_{2}\right) d \boldsymbol{k}_{123},
$$

is thus a fourth order correction of the frequency of oscillation, and

$$
\begin{gathered}
\Gamma^{l_{2}}\left(\boldsymbol{p}_{2}\right)=18 \pi(2 \pi)^{2} \sum_{s_{1} s_{2}} \int\left|L_{\boldsymbol{p}_{2} \boldsymbol{k}_{1} \boldsymbol{k}_{2} \boldsymbol{k}_{3}}^{l_{2} s_{1} s_{2} l_{2}}\right|^{2} q_{0}^{(2)-s_{1} s_{1}}\left(\boldsymbol{k}_{1}\right) q_{0}^{(2)-s_{2} s_{2}}\left(\boldsymbol{k}_{2}\right) \delta\left(\boldsymbol{k}_{1}+\boldsymbol{k}_{2}+\boldsymbol{k}_{3}-\boldsymbol{p}_{2}\right) \delta\left(\Omega_{\boldsymbol{p}_{2} \boldsymbol{k}_{1} \boldsymbol{k}_{2} \boldsymbol{k}_{3}}^{-l_{2} s_{1} s_{2} l_{2}}\right) d \boldsymbol{k}_{123} \\
-18 \pi(2 \pi)^{2} \sum_{s_{1} s_{2}} \int\left|L_{\boldsymbol{p}_{2} \boldsymbol{k}_{1} \boldsymbol{k}_{2} \boldsymbol{k}_{3}}^{l_{2} s_{1} s_{2}-l_{2}}\right|^{2} q_{0}^{(2)-s_{1} s_{1}}\left(\boldsymbol{k}_{1}\right) q_{0}^{(2)-s_{2} s_{2}}\left(\boldsymbol{k}_{2}\right) \delta\left(\boldsymbol{k}_{1}+\boldsymbol{k}_{2}+\boldsymbol{k}_{3}-\boldsymbol{p}_{2}\right) \delta\left(\Omega_{\boldsymbol{p}_{2} \boldsymbol{k}_{1} \boldsymbol{k}_{2} \boldsymbol{k}_{3}}^{-l_{2} s_{1} s_{2}-l_{2}}\right) d \boldsymbol{k}_{123}
\end{gathered}
$$

is a damping term, which is also present in the slow dynamics of higher order cumulant. It turns out that $\Gamma_{\boldsymbol{p}_{2}}^{l_{2}}$ becomes positive, which means that $q_{0}^{(2) l_{2} l_{2}}\left(\boldsymbol{p}_{2}\right)$ decays towards zero in time as well as the higher order cumulants.

\footnotetext{
${ }^{4}$ The general second order cumulant, $q_{2}^{(2) l_{1} l_{2}}\left(\boldsymbol{p}_{2}, t\right)$, corresponds to a $2 \times 2$ matrix, $l_{1} \& l_{2}$ being the indices of the matrix. By analogy we call $q_{2}^{(2) l l}(\boldsymbol{p}, t)$ the diagonal second order cumulant and $q_{2}^{(2)-l l}(\boldsymbol{p}, t)$ the off-diagonal second order cumulant.
} 
Finally, for the case $l_{1}=-l_{2}$ the resonant terms give

$$
\begin{aligned}
& F_{4}^{(2)-l_{2} l_{2}}\left(\boldsymbol{p}_{2}\right)= \epsilon^{4} 12 \pi(2 \pi)^{2}\left\{\sum_{s_{1} s_{2} s_{3}} \int\left|L_{\boldsymbol{p}_{2} \boldsymbol{k}_{1} \boldsymbol{k}_{2} \boldsymbol{k}_{3}}^{l_{2} s_{1} s_{2} s_{3}}\right|_{0}^{2} q_{0}^{(2)-s_{1} s_{1}}\left(\boldsymbol{k}_{1}\right) q_{0}^{(2)-s_{2} s_{2}}\left(\boldsymbol{k}_{2}\right) q_{0}^{(2)-s_{3} s_{3}}\left(\boldsymbol{k}_{3}\right)\right. \\
&\left.\times \delta\left(\boldsymbol{k}_{1}+\boldsymbol{k}_{2}+\boldsymbol{k}_{3}-\boldsymbol{p}_{2}\right) \delta\left(\Omega_{\boldsymbol{p}_{2} \boldsymbol{k}_{1} \boldsymbol{k}_{2} \boldsymbol{k}_{3}}^{-l_{2} s_{1} s_{2} s_{3}}\right) d \boldsymbol{k}_{123}\right\} \\
&- \epsilon^{4} 12 \pi(2 \pi)^{2}\left\{\sum_{s_{1} s_{2} s_{3}} \int\left|L_{\boldsymbol{p}_{2} \boldsymbol{k}_{1} \boldsymbol{k}_{2} \boldsymbol{k}_{3}}^{l_{2} s_{2} s_{2} s_{3}}\right|^{2} \mathcal{P}_{123}\left(\frac{s_{3}}{l_{2}}\right) q_{0}^{(2)-l_{2} l_{2}}\left(\boldsymbol{p}_{2}\right) q_{0}^{(2)-s_{1} s_{1}}\left(\boldsymbol{k}_{1}\right) q_{0}^{(2)-s_{2} s_{2}}\left(\boldsymbol{k}_{2}\right)\right. \\
&\left.\times \delta\left(\boldsymbol{k}_{1}+\boldsymbol{k}_{2}+\boldsymbol{k}_{3}-\boldsymbol{p}_{2}\right) \delta\left(\Omega_{\boldsymbol{p}_{2} \boldsymbol{k}_{1} \boldsymbol{k}_{2} \boldsymbol{k}_{3}}^{-l_{2} s_{2} s_{2} s_{3}}\right) d \boldsymbol{k}_{123}\right\}
\end{aligned}
$$

Since $F_{4}^{(2)-l_{2} l_{2}}\left(\boldsymbol{p}_{2}\right)=\frac{d}{d t} q_{0}^{(2) l_{1} l_{2}}\left(\boldsymbol{p}_{2}\right)$ one obtains a closed equation for the second order cumulants. This four-wave kinetic equation is the final equation responsible for the slow evolution of the system, where the energy transfer between modes is carried out through a resonant four-waves interaction process. Nevertheless, it is important to notice that, in general, non-uniformities will arise in $R^{(n)}$ at small or large scales ( $\boldsymbol{k} \rightarrow \infty$ or $\boldsymbol{k} \rightarrow 0$ respectively) that will break down the well ordered expansion. This can lead to intermittent behavior dominated by fully nonlinear solutions [4, 48]. We shall comeback to this point later in section 8.2. From this equation (49), together with the definition of the second order cumulant $Q^{(2)-s s}(\boldsymbol{k})=(2 \pi) n(s \boldsymbol{k})$ one can deduce the general kinetic equation:

$$
\begin{aligned}
\frac{d}{d t} n\left(l_{2} \boldsymbol{p}_{2}\right)= & 12 \pi l_{2} \epsilon^{4} \sum_{s_{1} s_{2} s_{3}} \int \mid J_{-\boldsymbol{p}_{2} \boldsymbol{k}_{1} \boldsymbol{k}_{2} \boldsymbol{k}_{3}}^{-\left.l_{2} s_{2}\right|^{2}} n\left(s_{1} \boldsymbol{k}_{1}\right) n\left(s_{2} \boldsymbol{k}_{2}\right) n\left(s_{3} \boldsymbol{k}_{3}\right) n\left(l_{2} \boldsymbol{p}_{2}\right)\left(\frac{l_{2}}{n\left(l_{2} \boldsymbol{p}_{2}\right)}-\frac{s_{1}}{n\left(s_{1} \boldsymbol{k}_{1}\right)}-\frac{s_{2}}{n\left(s_{2} \boldsymbol{k}_{2}\right)}-\frac{s_{3}}{n\left(s_{3} \boldsymbol{k}_{3}\right)}\right) \times \\
& \times \delta\left(\boldsymbol{k}_{1}+\boldsymbol{k}_{2}+\boldsymbol{k}_{3}-\boldsymbol{p}_{2}\right) \delta\left(l_{2} \omega\left(\boldsymbol{p}_{2}\right)-s_{1} \omega\left(\boldsymbol{k}_{1}\right)-s_{2} \omega\left(\boldsymbol{k}_{2}\right)-s_{3} \omega\left(\boldsymbol{k}_{3}\right)\right) d \boldsymbol{k}_{123},
\end{aligned}
$$

which becomes (20) if one assumes isotropy in the wave number distributions.

\section{Properties of the kinetic equation}

\subsection{The resonant manifold for the four waves interactions}

From the kinetic equation (20) one may see that the collisional term has two $\delta$-functions, which implies the conservation of the energy and the momentum respectively for every four waves interaction. The conservations of energy and momentum constrain the domain of integration to a manifold, named the resonant manifold (RM). In general, if the dimension of the system is $d$ the integration of the four wave collisional term is over a volume of dimension $3 d$. However the $\delta$-functions decreasing by $d+1$ the dimension of the total volume of integration, the resonant manifold of integration is of $2 d-1$ dimensions. In our case $d=2$ so that the resonant manifold of integration is of dimension 3 .

We need to analyze three different kinds of 4 -wave interactions, which are characterized in terms of the signs of $s_{1}, s_{2}$ and $s_{3}$ of the resonant condition : $l_{1} \omega\left(\boldsymbol{p}_{1}\right)+s_{1} \omega\left(\boldsymbol{k}_{1}\right)+s_{2} \omega\left(\boldsymbol{k}_{2}\right)+s_{3} \omega\left(\boldsymbol{k}_{3}\right)$. Without any loss of generality we can take $l_{1}=1$ and the three different cases are: firstly, when all signs $s_{1}, s_{2}, s_{3}$ are +1 , we denote this case as a process $4 \leftrightarrow 0$. Then, when two terms have a negative sign, which represents two waves decaying into other two, labeled as a $2 \leftrightarrow 2$ process. Finally, when one sign only is -1 , which represents a process of three waves decaying into a single one, labeled as $3 \leftrightarrow 1$. It should be noticed that $\boldsymbol{p}_{1}$ is not an integration variable, so that the $2 d-1$ resonant manifold should exist for an arbitrary value of $\boldsymbol{p}_{1}$.

Next we study these three processes in detail:

i) Case $4 \leftrightarrow 0$. In this case all terms in the energy conservation $\delta$-funtion have the same sign:

$$
\omega\left(\boldsymbol{p}_{1}\right)+\omega\left(\boldsymbol{k}_{1}\right)+\omega\left(\boldsymbol{k}_{2}\right)+\omega\left(\boldsymbol{k}_{3}\right)=0,
$$

because in general $\omega(\boldsymbol{k}) \geq 0$, one concludes that the only solution is given by $\omega\left(\boldsymbol{k}_{i}\right)=\omega\left(\boldsymbol{p}_{1}\right)=0$, so that there is in fact no manifold. This kind of interaction is not allowed at any dimension and one may eliminate it from the kinetic equation (20).

ii) Case $2 \leftrightarrow 2$ In this case the resonant manifold is defined by the following restrictions :

$$
\begin{array}{r}
\omega\left(\boldsymbol{p}_{1}\right)+\omega\left(\boldsymbol{k}_{1}\right)-\omega\left(\boldsymbol{k}_{2}\right)-\omega\left(\boldsymbol{k}_{3}\right)=0 \\
\boldsymbol{p}_{1}+\boldsymbol{k}_{1}-\boldsymbol{k}_{2}-\boldsymbol{k}_{3}=0
\end{array}
$$


One can realize that such a restriction is always satisfied over a $2 d-1$ manifold for any dispersion relation at any space dimension $d \geq 2$.

iii) Case $3 \leftrightarrow 1$.- One may consider without loss of generality

$$
\begin{array}{r}
\omega\left(\boldsymbol{p}_{1}\right)+\omega\left(\boldsymbol{k}_{1}\right)+\omega\left(\boldsymbol{k}_{2}\right)-\omega\left(\boldsymbol{k}_{3}\right)=0 \\
\boldsymbol{p}_{1}+\boldsymbol{k}_{1}+\boldsymbol{k}_{2}-\boldsymbol{k}_{3}=0 .
\end{array}
$$

This case is particularly interesting, because, depending on the dispersion relation this resonant manifold could be empty and the total wave action of the system conserved. However, we will show that for an elastic plate this is not the case and the total wave number is not conserved.

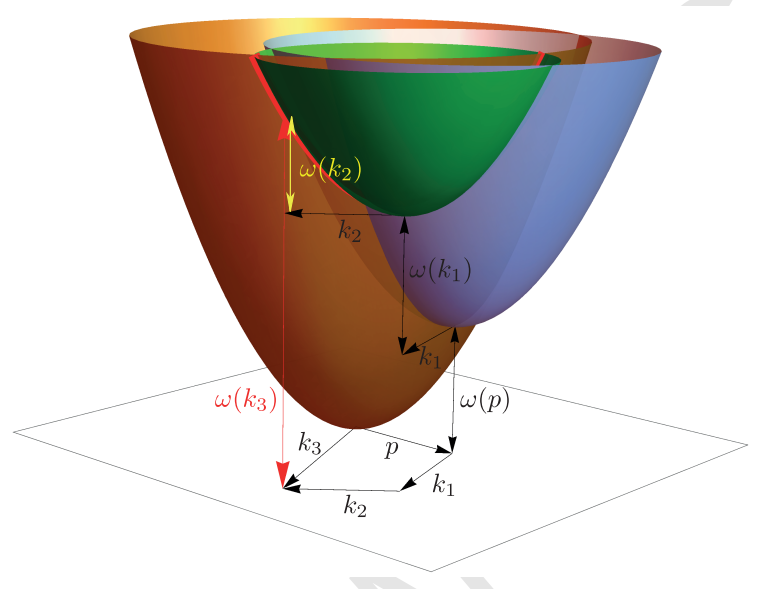

Figure 1: The plot represents the intersection of the surfaces for a parabolic dispersion relation $\omega(\boldsymbol{k})=\frac{1}{2}|\boldsymbol{k}|^{2}$. This is the case of a decaying situation in the $3 \leftrightarrow 1$.

To visualize the existence of the resonant manifold it is convenient to do geometrically. The dispersion relation is isotropic depending only on the modulus of $|\boldsymbol{k}|$, therefore the function $\omega(k)$ determines a paraboloid of revolution in the three space dimensions $\left(k_{x}, k_{y}, \omega\right)$. Take now a point $A$ on the surface with coordinates $\left(p_{1 x}, p_{1 y}, \omega\left(p_{1}\right)\right)$ and build a new surface $\omega(k)$ with $A$ as its origin. In the same way, take now another point $B$ over the new surface with coordinates $\left(p_{1 x}+k_{1 x}, p_{1 y}+k_{1 y}, \omega\left(p_{1}\right)+\omega\left(k_{1}\right)\right)$ and we build again a surface $\omega(k)$ but with an origin at $B$. Fig. 1 shows this geometric construction and we can see that for any wavenumber $\boldsymbol{p}_{1}$ and $\boldsymbol{k}_{1}$, an intersection exists between the revolution paraboloid of origin $O$ with the one that has B for origin, satisfying therefore precisely the conditions imposed in (52). As expected, this problem has 5 degrees of freedom, but one of this variable $\boldsymbol{p}_{1}$ is not integrated, and so, the domain of integration becomes a three dimensional manifold.

\subsection{Conserved quantities}

As the usual Boltzmann equation for diluted gases, (20) conserves formally the momentum and the energy density ${ }^{5}$, and the dynamics exhibits irreversibility because of the existence of a H-Theorem. Here we develop in details these three statements.

\subsubsection{Energy conservation}

Firstly, we can show that the kinetic energy per unit area

$$
\mathcal{E}=\frac{1}{2} \sum_{l_{1}} \int \omega\left(l_{1} \boldsymbol{p}_{1}\right) n\left(l_{1} \boldsymbol{p}_{1}, t\right) d \boldsymbol{p}_{1}
$$

is conserved.

\footnotetext{
${ }^{5}$ Here we stands by energy for the lowest order in $\epsilon^{2}$ of the full energy (28). The full energy is not preserved by the kinetics, as in diluted gases the conserved energy by Boltzmann equation preserves the kinetic energy of the gas only.
} 
Proof.- The proof is the same as for the usual Boltzmann equation, computing first the time derivative

$$
\frac{d \mathcal{E}}{d t}=\frac{1}{2} \sum_{l_{1}} \int \omega\left(l_{1} \boldsymbol{p}_{1}\right) \frac{d}{d t} n\left(l_{1} \boldsymbol{p}_{1}, t\right) d \boldsymbol{p}_{1}=\frac{1}{2} \sum_{l_{1} s_{1} s_{2} s_{3}} \int l_{1} \omega\left(l_{1} \boldsymbol{p}_{1}\right) \mathcal{C}_{\boldsymbol{p}_{1} \boldsymbol{k}_{1} \boldsymbol{k}_{2} \boldsymbol{k}_{3}}^{l_{1} s_{1} s_{2} s_{3}} d \boldsymbol{p}_{1} d \boldsymbol{k}_{123}
$$

where in the third equality the full kinetic equation (50) has been used. We have introduced here the collisional operator, which is completely symmetric in the index $\left\{l_{1}, s_{1}, s_{2}, s_{3}\right\}$ and $\left\{\boldsymbol{p}_{1}, \boldsymbol{k}_{1}, \boldsymbol{k}_{2}, \boldsymbol{k}_{3}\right\}$, following:

$$
\begin{aligned}
\mathcal{C}_{\boldsymbol{p}_{1} \boldsymbol{k}_{1} \boldsymbol{k}_{2} \boldsymbol{k}_{3}}^{l_{1} s_{1} s_{2} s_{3}}= & 12 \pi\left|J_{\boldsymbol{p}_{1} \boldsymbol{k}_{1} \boldsymbol{k}_{2} \boldsymbol{k}_{3}}^{l_{1} s_{1} s_{2} s_{3}}\right|^{2} n\left(s_{1} \boldsymbol{k}_{1}\right) n\left(s_{2} \boldsymbol{k}_{2}\right) n\left(s_{3} \boldsymbol{k}_{3}\right) n\left(l_{1} \boldsymbol{p}_{1}\right)\left(l_{1} \frac{1}{n\left(l_{1} \boldsymbol{p}_{1}\right)}+s_{1} \frac{1}{n\left(s_{1} \boldsymbol{k}_{1}\right)}+s_{2} \frac{1}{n\left(s_{2} \boldsymbol{k}_{2}\right)}+s_{3} \frac{1}{n\left(s_{3} \boldsymbol{k}_{3}\right)}\right) \\
& \times \delta\left(l_{1} \omega\left(l_{1} \boldsymbol{p}_{1}\right)+s_{1} \omega\left(s_{1} \boldsymbol{k}_{1}\right)+s_{2} \omega\left(s_{2} \boldsymbol{k}_{2}\right)+s_{3} \omega\left(s_{3} \boldsymbol{k}_{3}\right)\right) \delta\left(\boldsymbol{k}_{1}+\boldsymbol{k}_{2}+\boldsymbol{k}_{3}+\boldsymbol{p}_{1}\right) .
\end{aligned}
$$

Repeating four times each term, then exchanging integrals and re-labeling the index leads to:

$$
\frac{d \mathcal{E}}{d t}=\frac{1}{8} \sum_{l_{1} s_{1} s_{2} s_{3}} \int\left(l_{1} \omega\left(l_{1} \boldsymbol{p}_{1}\right)+s_{1} \omega\left(s_{1} \boldsymbol{k}_{1}\right)+s_{2} \omega\left(s_{2} \boldsymbol{k}_{2}\right)+s_{3} \omega\left(s_{3} \boldsymbol{k}_{3}\right)\right) \mathcal{C}_{\boldsymbol{p}_{1} \boldsymbol{k}_{1} \boldsymbol{k}_{2} \boldsymbol{k}_{3}}^{l_{1} s_{1} s_{2} s_{3}} d \boldsymbol{p}_{1} d \boldsymbol{k}_{123}=0 .
$$

which is exactly zero because of the $\delta\left(l_{1} \omega\left(l_{1} \boldsymbol{p}_{1}\right)+s_{1} \omega\left(s_{1} \boldsymbol{k}_{1}\right)+s_{2} \omega\left(s_{2} \boldsymbol{k}_{2}\right)+s_{3} \omega\left(s_{3} \boldsymbol{k}_{3}\right)\right)$ of the kinetic equation.

Remark 1.- Notice that isotropy: $\omega\left(l_{1} \boldsymbol{p}_{1}\right)=\omega\left(-l_{1} \boldsymbol{p}_{1}\right)$ and $n\left(l_{1} \boldsymbol{p}_{1}, t\right)=n\left(-l_{1} \boldsymbol{p}_{1}, t\right)$ is not required in the formal proof.

Remark 2.- This proof needs exchange of integrals, therefore it requires convergence of any simple integral before any exchange can be done [4] (Fubini's theorem).

\subsubsection{Momentum conservation}

Similarly, the total momentum per unit area

$$
\boldsymbol{J}=\frac{1}{2} \sum_{l_{1}} \int l_{1} \boldsymbol{p}_{1} n\left(l_{1} \boldsymbol{p}_{1}, t\right) d \boldsymbol{p}_{1}
$$

is conserved. The proof is in the same vein than for the conservation of energy (not detailed here).

Remark 1.- Because of the existence of the $3 \leftrightarrow 1$ processes the total wave action

$$
\mathcal{N}=\frac{1}{2} \sum_{l_{1}} \int n\left(l_{1} \boldsymbol{p}_{1}, t\right) d \boldsymbol{p}_{1}
$$

is not conserved. If one derives this quantity

$$
\frac{d \mathcal{N}}{d t}=\frac{1}{8} \sum_{l_{1} s_{1} s_{2} s_{3}}\left(l_{1}+s_{1}+s_{2}+s_{3}\right) \mathcal{C}_{\boldsymbol{p}_{1} \boldsymbol{k}_{1} \boldsymbol{k}_{2} \boldsymbol{k}_{3}}^{l_{1} s_{1} s_{2} s_{3}} d \boldsymbol{p}_{1} d \boldsymbol{k}_{123}
$$

the only terms that survive in the sum are those corresponding to the $3 \leftrightarrow 1$ processes, hence

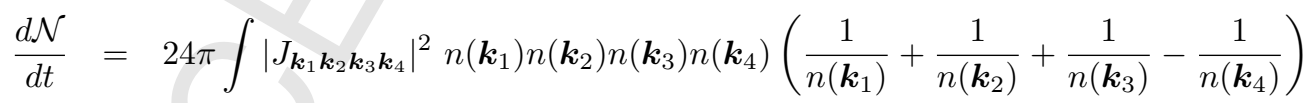

$$
\begin{aligned}
& \times \delta\left(\omega\left(\boldsymbol{k}_{1}\right)+\omega\left(\boldsymbol{k}_{2}\right)+\omega\left(\boldsymbol{k}_{3}\right)-\omega\left(\boldsymbol{k}_{4}\right)\right) \delta\left(\boldsymbol{k}_{1}+\boldsymbol{k}_{2}+\boldsymbol{k}_{3}+\boldsymbol{k}_{4}\right) d \boldsymbol{k}_{1234} .
\end{aligned}
$$

We notice then that because of the existence of the $3 \leftrightarrow 1$ processes the wave action is not conserved formally. However, the wave action reaches a steady state for the equilibrium distribution (Rayleigh-Jeans) as well as for the energy cascade spectrum, because in these cases the right hand side of eq. (59) vanishes. 


\subsubsection{H-Theorem}

Although the original equations are reversible, the kinetic theory exhibits an irreversible dynamics towards thermodynamical equilibrium for an isolated system. In practice, in systems with a large number of degrees of freedom, the irreversibility can be seen as a result of chaotic behavior together with some small but non zero noise (which always exists). A positive Lyapunov exponent, characteristic of chaotic system, is then able to amplify any extremely small noise. The irreversibility is linked to the loss of the informations contained in the phases of the Fourier amplitudes, which disappear in the averaging process over the ensemble. Mathematically, the loss of exact reversibility of the original equations is produced in the multi-scale perturbation expansion. More specifically, it is the result of taking the long time limit over the linear fast time, keeping the slower nonlinear time scale constant.

In the context of kinetic theory the irreversibility and the evolution towards equilibrium can be expressed by a H-theorem, that we state next. Let

$$
\mathcal{S}(t)=\frac{1}{2} \sum_{l_{1}} \int \log \left[n\left(l_{1} \boldsymbol{p}_{1}, t\right)\right] d \boldsymbol{p}_{1}
$$

be the non-equilibrium entropy, then one can show that

$$
d \mathcal{S} / d t \geq 0
$$

for increasing time.

Proof.- Consider the time derivative of the entropy defined above

$$
\frac{d S}{d t}=\frac{1}{2} \sum_{l_{1}} \int \frac{1}{n\left(l_{1} \boldsymbol{p}_{1}, t\right)} \frac{d n\left(l_{1} \boldsymbol{p}_{1}, t\right)}{d t} d \boldsymbol{p}
$$

proceeding again in the same way as above, one splits the four terms, leading to

$$
\frac{d \mathcal{S}}{d t}=\frac{1}{8} \sum_{l_{1} s_{1} s_{2} s_{3}} \int\left(l_{1} \frac{1}{n\left(l_{1} \boldsymbol{p}_{1}, t\right)}+s_{1} \frac{1}{n\left(s_{1} \boldsymbol{k}_{1}, t\right)}+s_{2} \frac{1}{n\left(s_{2} \boldsymbol{k}_{2}, t\right)}+s_{3} \frac{1}{n\left(s_{3} \boldsymbol{k}_{3}, t\right)}\right) \mathcal{C}_{\boldsymbol{p}_{1} \boldsymbol{k}_{1} \boldsymbol{k}_{2} \boldsymbol{k}_{3}}^{l_{1} s_{1} s_{2} s_{3}} d \boldsymbol{p}_{1} d \boldsymbol{k}_{123} \geq 0
$$

Using (55), one can see that the integrand is always positive or at least zero, so that the entropy of a closed wave system can only increase or remain constant.

The thermodynamical equilibrium corresponds to the case of $\frac{d S}{d t} \equiv 0$ which is satisfied only by the functional relation

$$
\left(l_{1} \frac{1}{n\left(l_{1} \boldsymbol{p}_{1}\right)}+s_{1} \frac{1}{n\left(s_{1} \boldsymbol{k}_{1}\right)}+s_{2} \frac{1}{n\left(s_{2} \boldsymbol{k}_{2}\right)}+s_{3} \frac{1}{n\left(s_{3} \boldsymbol{k}_{3}\right)}\right)=0
$$

for $\boldsymbol{p}_{1}, \boldsymbol{k}_{1}, \boldsymbol{k}_{2}$ and $\boldsymbol{k}_{3}$, in the resonant manifold. Because of the resonant conditions, the solution to this functional equation is a linear combination of $\omega(\boldsymbol{k})$ and $\boldsymbol{k}$, that is: $n(\boldsymbol{k})^{-1}=\frac{1}{T} \omega(\boldsymbol{k})+\frac{1}{T} \boldsymbol{u} \cdot \boldsymbol{k}$ with $T$ and $\boldsymbol{u}$ two constant parameters. This solution corresponds to the thermodynamical equilibrium and it is known as the Rayleigh-Jeans distribution [49, 50]:

$$
n(\boldsymbol{k})=\frac{T}{\omega(\boldsymbol{k})+\boldsymbol{u} \cdot \boldsymbol{k}} .
$$

Similarly, this Rayleigh-Jeans distribution may be deduced by maximizing the entropy with the corresponding constrains of constant energy and momentum [51]. Using the technique of Lagrange multipliers (below $T$ and $\boldsymbol{u}$ are Lagrange multipliers) and writing the extremum of the entropy condition yielding

$$
\frac{\delta}{\delta n(\boldsymbol{k})}\left(\mathcal{S}-\frac{1}{T} \mathcal{E}-\frac{1}{T} \boldsymbol{u} \cdot \boldsymbol{J}\right)=0
$$

one readily gets $(62)$.

Remark 1.- As we shall see below, the energy flux of this solution is identically zero. The wave system at equilibrium presents thus no energy, neither momentum flux. This is because the detailed balance condition at equilibrium forbids any net flux of energy or other quantity. 
Remark 2.- In general in our case the total initial momentum $\boldsymbol{J}$ is zero, the resulting spectrum is isotropic so that $\boldsymbol{u}=0$. The Rayleigh-Jeans equilibrium distribution reads then:

$$
n_{\boldsymbol{k}}^{e q}=\frac{T}{\omega_{k}}
$$

Where $T$ is called, by analogy with thermodynamics, the temperature which is naturally related to the initial energy by $\mathcal{E}_{0}=\int \omega_{k} n_{k}^{e q} d^{2} \boldsymbol{k}=T \int d^{2} \boldsymbol{k}$, the quantity $\int d^{2} \boldsymbol{k}$ being the number of degrees of freedom per surface unit. Therefore each degree of freedom contains the same energy: $T$. Naturally, for an infinite system this number diverges (as well as the energy). This classical Rayleigh-Jeans catastrophe is always suppressed due to some physical cut-off, which corresponds to dissipation processes at small scales. Numerical simulations on regular grids provide also a natural cut-off $k_{c}=\pi / d x$, where $d x$ is the mesh size, which gives $\mathcal{E}_{0}=\pi T k_{c}^{2}$ for a large system.

\subsection{Kinetic equation for an isotropic spectra}

In this section we consider the kinetic equation (20) for the special case of an isotropic distribution $n(\boldsymbol{p})=n(|\boldsymbol{p}|)=n_{p}$ (where $p=|\boldsymbol{p}|$ ), an hypothesis that is often done in wave turbulence. Thus, because of the isotropic dispersion relation $\omega(\boldsymbol{p})=\omega(|\boldsymbol{p}|)=\omega_{p}$, the spectrum $n_{p}$ is simply a function of the frequency $\omega_{p}$, something which is more useful for the comparison with experiments.

Multiplying (20) by $\frac{p}{\omega_{p}^{\prime}} d \varphi_{p}$, where $\omega_{p}^{\prime}=d \omega_{p} / d p$, and integrating over the polar variables $d \varphi_{p} d \varphi_{k_{1}} d \varphi_{k_{2}} d \varphi_{k_{3}}$ in $[0,2 \pi]$, the resulting kinetic equation reads:

$$
\frac{d}{d t}\left(\frac{2 \pi p}{\omega_{p}^{\prime}} n_{p}\right)=12 \pi \sum_{s_{1} s_{2} s_{3}} \int_{\mathcal{D}} S_{\omega_{p} \omega_{1} \omega_{2} \omega_{3}} n_{1} n_{2} n_{3} n_{p}\left(\frac{1}{n_{p}}+s_{1} \frac{1}{n_{1}}+s_{2} \frac{1}{n_{2}}+s_{3} \frac{1}{n_{3}}\right) \delta\left(\omega_{p}+s_{1} \omega_{1}+s_{2} \omega_{2}+s_{3} \omega_{3}\right) d \omega_{123}
$$

The domain of integration, $\mathcal{D}$, is defined in such a way that each frequency is positive, i.e. $\omega_{1}, \omega_{2}, \omega_{3}>0$. For simplicity we use the notation $n_{k_{i}}=n_{i}$ and $\omega_{k_{i}}=\omega_{i}$, and the scattering matrix, which is completely symmetric, reads

$$
S_{\omega_{p} \omega_{1} \omega_{2} \omega_{3}}=\frac{p}{\omega_{p}^{\prime}} \frac{k_{1}}{\omega_{1}^{\prime}} \frac{k_{2}}{\omega_{2}^{\prime}} \frac{k_{3}}{\omega_{3}^{\prime}}\left(\int_{0}^{2 \pi}\left|J_{\boldsymbol{p k}_{1} \boldsymbol{k}_{2} \boldsymbol{k}_{3}}^{+s_{1} s_{2} s_{3}}\right|^{2} \delta\left(\boldsymbol{k}_{1}+\boldsymbol{k}_{2}+\boldsymbol{k}_{3}+\boldsymbol{p}\right) d \varphi_{p} d \varphi_{k_{1}} d \varphi_{k_{2}} d \varphi_{k_{3}}\right) .
$$

Obtain an explicit form of this isotropic scattering matrix it is complicated. However, an explicit expression for an upper and lower bound to this scattering matrix can be obtained (see section 6.2 or Appendix 10.7).

Remark 1.- It maybe useful to define the frequency spectrum

$$
N\left(\omega_{p}\right)=2 \pi \frac{p}{\omega_{p}^{\prime}} n_{p}
$$

which is naturally normalized since $\int_{0}^{\infty} N(\omega) d \omega=\int n(\boldsymbol{p}) d \boldsymbol{p}$.

Remark 2.- Because the dispersion relation has an homogeneity degree 2, $\omega(\lambda \boldsymbol{k})=\lambda^{2} \omega(\boldsymbol{k})$, and because $J_{\lambda \boldsymbol{p} \lambda \boldsymbol{k}_{1} \lambda \boldsymbol{k}_{2} \lambda \boldsymbol{k}_{3}}=$ $J_{p \boldsymbol{k}_{1} \boldsymbol{k}_{2} \boldsymbol{k}_{3}}$ does not scale in $k$, the $S$-matrix has an homogeneity

$$
S_{\lambda \omega_{p} \lambda \omega_{1} \lambda \omega_{2} \lambda \omega_{3}}=\lambda^{-1} S_{\omega_{p} \omega_{1} \omega_{2} \omega_{3}} .
$$

Equation (64) contains seven different terms, four of them of the type $3 \leftrightarrow 1$ and the three other terms of the type $2 \leftrightarrow 2$. These terms may be collected in two distinct collision integrals:

$$
\frac{d}{d t}\left(\frac{2 \pi p}{\omega_{p}^{\prime}} n_{p}\right)=\operatorname{Coll}_{3 \leftrightarrow 1}[n]+\operatorname{Coll}_{2 \leftrightarrow 2}[n]
$$


Here $\mathrm{Coll}_{3 \leftrightarrow 1}[n]$ collects the wave interaction of the type $3 \leftrightarrow 1$. Using the symmetries of $S_{\omega_{p} \omega_{1} \omega_{2} \omega_{3}}^{+s_{3}}$, this collision integral reads:

$$
\begin{aligned}
& \operatorname{Coll}_{3 \leftrightarrow 1}[n]=12 \pi \times\left[\int_{\mathcal{D}} S_{\omega_{p} \omega_{1} \omega_{2} \omega_{3}} n_{1} n_{2} n_{3} n_{p}\left(\frac{1}{n_{p}}-\frac{1}{n_{1}}-\frac{1}{n_{2}}-\frac{1}{n_{3}}\right) \delta\left(\omega_{p}-\omega_{1}-\omega_{2}-\omega_{3}\right) d \omega_{123}\right. \\
& +\int_{\mathcal{D}} S_{\omega_{p} \omega_{1} \omega_{2} \omega_{3}} n_{p} n_{1} n_{2} n_{3}\left(\frac{1}{n_{p}}-\frac{1}{n_{1}}+\frac{1}{n_{2}}+\frac{1}{n_{3}}\right) \delta\left(\omega_{1}-\omega_{p}-\omega_{2}-\omega_{3}\right) d \omega_{123} \\
& +\int_{\mathcal{D}} S_{\omega_{p} \omega_{1} \omega_{2} \omega_{3}} n_{p} n_{1} n_{2} n_{3}\left(\frac{1}{n_{p}}+\frac{1}{n_{1}}-\frac{1}{n_{2}}+\frac{1}{n_{3}}\right) \delta\left(\omega_{2}-\omega_{p}-\omega_{1}-\omega_{3}\right) d \omega_{123} \\
& \left.+\int_{\mathcal{D}} S_{\omega_{p} \omega_{1} \omega_{2} \omega_{3}} n_{p} n_{1} n_{2} n_{3}\left(\frac{1}{n_{p}}+\frac{1}{n_{1}}+\frac{1}{n_{2}}-\frac{1}{n_{3}}\right) \delta\left(\omega_{3}-\omega_{p}-\omega_{2}-\omega_{1}\right) d \omega_{123}\right] .
\end{aligned}
$$

These four terms could be easily collected into a single one by relabelling the integration variables. However, we keep the more symmetric form (68), because it will be useful in the calculations. On the other hand the collision integral $\mathrm{Coll}_{2 \leftrightarrow 2}[n]$ gathers the terms of the $2 \leftrightarrow 2$ wave interaction. These term can be re-arranged with an adequate change of variables, yielding

$$
\operatorname{Coll}_{2 \leftrightarrow 2}[n]=36 \pi \int_{\mathcal{D}} S_{\omega_{p} \omega_{1} \omega_{2} \omega_{3}} n_{1} n_{2} n_{3} n_{p}\left(\frac{1}{n_{p}}+\frac{1}{n_{1}}-\frac{1}{n_{2}}-\frac{1}{n_{3}}\right) \delta\left(\omega_{p}+\omega_{1}-\omega_{2}-\omega_{3}\right) d \omega_{123} .
$$

It is important to emphasize that one can deduce from the kinetic equation (67) a conservation equation for the energy spectrum $E(\omega, t)=\omega N(\omega, t)$ in the form

$$
\frac{\partial E(\omega, t)}{\partial t}+\frac{\partial P(\omega, t)}{\partial \omega}=0
$$

where $P(\omega, t)$ is the energy flux in the frequency space $\omega$ given by the relation

$$
\frac{\partial P(\omega, t)}{\partial \omega}=-\omega\left(\operatorname{Coll}_{3 \leftrightarrow 1}[n]+\operatorname{Coll}_{2 \leftrightarrow 2}[n]\right) .
$$

Equation (70) satisfies two types of isotropic stationary solutions:

i) No flux solution.- The case whenever the energy flux is identically null, $P=0$, which corresponds to the equilibrium solution, the so called Rayleigh-Jeans distribution [49, 50].

ii) Constant flux solution.- If $P(\omega)=P \neq 0$ is constant inside an inertial range, a stationary solution satisfying (71) with a constant energy flux can exist. These kinds of out-of-equilibrium solutions are usually, but not always, power law distributions, in which case are known as Kolmogorov-Zakharov spectra. To obtain these kinds of turbulent spectra it is formally necessary to inject energy (mostly) at frequencies below some injection range $\omega_{i}$ and to dissipate it (mostly) at frequencies larger than some dissipation scale $\omega_{d}$.

In principle, the injection can be modeled as a function $\mathcal{I}$ independent of the plate deformation $\zeta$ which vanishes for $\omega>\omega_{i}$ and the dissipation as a function $\mathcal{D}$ linear on the deformation velocity of the plate $\dot{\zeta}$ which vanishes for $\omega<\omega_{d}$. These functions can be added to the Föppl-von Kármán equation (1) to describe the injection and dissipation of energy (see section (7) for an example).

The kinetic equation under injection and dissipation could be derived in principle from the wave dynamical equation considering that injection and dissipation take place in time scales similar to the nonlinear transfer. The kinetic equation then should read

$$
\frac{d}{d t} N(\omega)=\operatorname{Coll}_{3 \leftrightarrow 1}[n]+\operatorname{Coll}_{2 \leftrightarrow 2}[n]+\Lambda(\omega)
$$

where $\Lambda(\omega)$ depends on the functions $\mathcal{I}$ and $\mathcal{D}$ and on the plate deformation. The explicit expression of $\Lambda$ has not been determined to the best of our knowledge and will be considered elsewhere. However it is not relevant as long as it vanishes within the transparency window $\left[\omega_{i}, \omega_{d}\right]$.

The properties on the collisional terms (56) and (61) implies that for a stationary solution $\frac{d}{d t} N(\omega)=0$ to exist, the $\Lambda$ function has to satisfy the following restrictions:

$$
\begin{aligned}
\int_{0}^{\infty} \omega \Lambda(\omega) d \omega & =0 \\
\int_{0}^{\infty} \frac{\Lambda}{n(\omega)} d \omega & \leq 0
\end{aligned}
$$


The condition (74) has a simple interpretation: out-equilibrium stationary solutions have less entropy than equilibrium solutions. Since the spectrum $n(\omega)$ is a positive quantity the constrains (74) and (73) implies that $\Lambda$ must change its sign. The $\Lambda>0$ zone corresponds then to the injection scale (usually at large scale or small frequency), while the $\Lambda<0$ corresponds to dissipation usually at small scales or large frequencies. If the interactions between the modes are local and the inertial range is large enough $\omega_{i} \ll \omega_{d}$, one can take approximately $\omega_{i} \rightarrow 0$ and $\omega_{d} \rightarrow \infty$ and look for turbulent stationary solutions to the conservative kinetic equation (67) (see section 6). Under such simplification, the injection and the dissipation take place at the boundary of the frequency domain i.e. $\lim _{\omega_{i} \rightarrow 0} P\left(\omega_{i}, t\right) \neq 0$ but $P(\omega=0, t)=0$. Therefore, the out of equilibrium solutions satisfies

$$
P(\omega, t)=-\int_{0}^{\omega} \omega\left(\operatorname{Coll}_{3 \leftrightarrow 1}[n]+\operatorname{Coll}_{2 \leftrightarrow 2}[n]\right) d \omega=P \neq 0 .
$$

These formula indicates that the energy is injected at $\omega=0$ only.

Moreover, from (72) and (73) one concludes that the energy flux may be defined through:

$$
P=-\int_{0}^{\omega_{d}} \omega \Lambda(\omega) d \omega=\int_{\omega_{d}}^{\infty} \omega \Lambda(\omega) d \omega
$$

where $\omega_{d}$ is a dissipation scale (see eq. (97) below).

\section{Kolmogorov Spectra}

\subsection{Zakharov-type of solutions}

In this section we derive precisely the exact stationary non zero flux solutions of the kinetic equation. These types of solutions were first obtained by V.E. Zakharov in the context of Langumir oscillations in plasma physics [8]. Essentially we consider both collisional integrals $\operatorname{Coll}_{3 \leftrightarrow 1}[n](68)$ and $\operatorname{Coll}_{2 \leftrightarrow 2}[n](69)$ acting on a power law type of solutions of the form $n_{p}=K \omega_{p}^{-x}$, where $K$ is a constant pre-factor which will be determined later in terms of the energy flux $P$. Although the collisional integral $C o l l_{2 \leftrightarrow 2}$, has been considered extensively in the literature for different context $[3,8]$, the case of $\operatorname{Coll}_{3 \leftrightarrow 1}[n]$ is definitely less known and to our knowledge, no reference exists prior to our work on plate turbulence [19]. Therefore, we will focus here principally on the calculations for this latter case.

Let us introduce a spectrum $n_{p}=K \omega_{p}^{-x}$ into the collisional terms $\operatorname{Coll}_{3 \leftrightarrow 1}[n]$ that is composed by four distinct contributions. The first term reads

$$
12 \pi K^{3} \int_{O} S_{\omega_{p} \omega_{1} \omega_{2} \omega_{3}}\left(\omega_{p} \omega_{1} \omega_{2} \omega_{3}\right)^{-x}\left(\omega_{p}^{x}-\omega_{1}^{x}-\omega_{2}^{x}-\omega_{3}^{x}\right) \delta\left(\omega_{p}-\omega_{1}-\omega_{2}-\omega_{3}\right) d \omega_{123}
$$

where the domain of integration $O$ corresponds to $\omega_{1}=\omega_{p}-\omega_{2}-\omega_{3} \geq 0$, as located specifically in Fig. 2. The three others terms exhibit the same structure:

$$
12 \pi K^{3} \int_{\mathcal{D}} S_{\omega_{p} \omega_{1} \omega_{2} \omega_{3}}\left(\omega_{1} \omega_{2} \omega_{3} \omega_{p}\right)^{-x}\left(\omega_{p}^{x}-\omega_{1}^{x}+\omega_{2}^{x}+\omega_{3}^{x}\right) \delta\left(\omega_{1}-\omega_{p}-\omega_{2}-\omega_{3}\right) d \omega_{123},
$$

obtained then by the permutations of $1 \leftrightarrow 2$ and $1 \leftrightarrow 3$. In the equation (77), the domain of integration $\mathcal{D}$ is the whole upper-right quadrant $\omega_{2}, \omega_{3} \geq 0,(\mathcal{D}=O \cup I \cup I I \cup I I I \cup I V$ in Fig. 2).

After the Zakharov's transformation [3,8] (see Appendix 10.6 for details) one readily gets:

$$
\operatorname{Coll}_{3 \leftrightarrow 1}[n]=K^{3} \omega_{p}^{-3 x+1} I_{3 \leftrightarrow 1}^{(1)}(x),
$$

where $I_{3 \leftrightarrow 1}^{(1)}(x)$ is a pure function of $x$ defined by

$$
I_{3 \leftrightarrow 1}^{(1)}(x)=12 \pi \int_{O^{\prime}} S_{1 u_{1} u_{2} u_{3}}\left(u_{1} u_{2} u_{3}\right)^{-x}\left(1-u_{1}^{x}-u_{2}^{x}-u_{3}^{x}\right)\left(1-u_{1}^{3 x-2}-u_{2}^{3 x-2}-u_{3}^{3 x-2}\right) \delta\left(1-u_{1}-u_{2}-u_{3}\right) d u_{123} .
$$

and $O^{\prime}$, is the domain $O$ rescaled by $\omega_{p}$. More precisely, it is the triangle defined by $u_{2}=0, u_{3}=0$ and $u_{2}+u_{3}=1$.

A similar calculation can be performed for the collisional integral (69), as presented in the Appendix 10.6, leading to

$$
\operatorname{Coll}_{2 \leftrightarrow 2}[n]=K^{3} \omega_{p}^{-3 x+1} I_{2 \leftrightarrow 2}^{(1)}(x),
$$




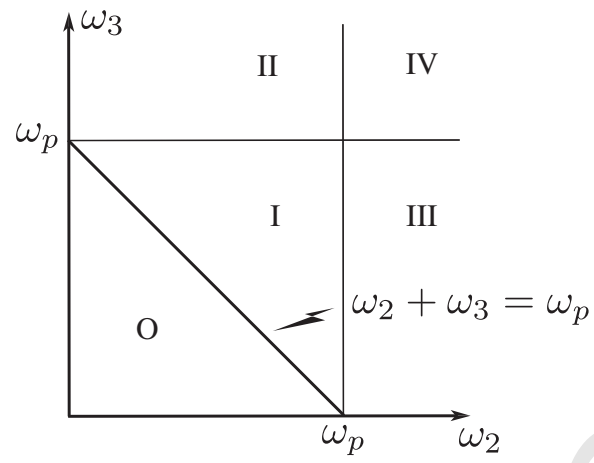

Figure 2: Relevant domains of integration in the plane $\left(\omega_{2}, \omega_{3}\right)$. The diagonal line represents $\omega_{2}+\omega_{3}=\omega_{p}$.

where $I_{2 \leftrightarrow 2}^{(1)}(x)$ is also a pure function of $x$, yielding

$$
I_{2 \leftrightarrow 2}^{(1)}(x)=36 \pi \int_{I^{\prime}} S_{1 u_{1} u_{2} u_{3}}\left(u_{1} u_{2} u_{3}\right)^{-x}\left(1+u_{1}^{x}-u_{2}^{x}-u_{3}^{x}\right)\left(1+u_{1}^{3 x-2}-u_{2}^{3 x-2}-u_{3}^{3 x-2}\right) \delta\left(1+u_{1}-u_{2}-u_{3}\right) d u_{123},
$$

and where, as before, $I^{\prime}$ is the rescaled domain deduced from the domain $I$ of Fig. 2. From now on, we seek for which values of $x$ these two integrals vanish. From (79) and (81) one sees immediately that the collisional integrals vanish both exactly for $x=1$, because of the first bracket in (79) and in (81). This stationary spectrum corresponds in fact to the Rayleigh-Jeans spectrum (62) already described above. Furthermore, vanishing the second bracket in the integrals selects usually the KZ spectra solutions. However, the second brackets in both integrals $I_{2 \leftrightarrow 2}^{(1)}(x)$ and $I_{3 \leftrightarrow 1}^{(1)}(x)$ vanish also for $x=1$. Therefore, both $I_{3 \leftrightarrow 1}^{(1)}(x)$ and $I_{2 \leftrightarrow 2}^{(1)}(x)$ have a double zero at $x=1$, indicating that these functions behave as $\sim(x-1)^{2}$ close to $x=1$. More physically, this mathematical analysis tells us that the Rayleigh-Jeans and the Kolmogorov-Zakharov spectra coincides. Therefore the energy flux is necessarily null, because $x=1$ vanishes exactly the r.h.s of the entropy production equation (61). Thus $x=1$ can only represent the equilibrium solution that exhibits zero flux. This degeneracy requires an extra effort in the computation of the Kolmogorov-Zakharov spectrum as we shall see in Section 6.3.

We end this section with the following remarks. The collisional integral $I_{2 \leftrightarrow 2}^{(1)}(x)$ alone vanishes also exactly for $x=0$ and $x=2 / 3$. The first one $(x=0)$ corresponds to an uniform spectrum that describes the equipartition of wave action and the second one $(x=2 / 3)$ has the significance of a wave action cascade, by analogy with the cases of gravity surface waves [7, 18] and nonlinear optics $[10,11,12]$. This latter solution would correspond to an inverse cascade of wave action with a constant flux but recall that it cannot exist because of the $I_{3 \leftrightarrow 1}^{(1)}(x)$ that does not vanish for $x=2 / 3$.

\subsection{Locality of the interactions.}

The only physically consistent Kolmogorov spectra are the ones that provide convergent results for the collisional integrals. If the collisional integrals $I_{3 \leftrightarrow 1}^{(1)}(79)$ and $I_{2 \leftrightarrow 2}^{(1)}(81)$ are convergent for a given $x$, it is said that the solution $n_{k} \sim k^{-2 x}$ is a local spectrum. However, if the integrals diverge, then the spectrum $n_{k} \sim k^{-2 x}$ is not a suitable solution.

In the following, we check the convergence criteria for the collision integrals $I_{3 \leftrightarrow 1}^{(1)}(x)$ and $I_{2 \leftrightarrow 2}^{(1)}(x)$ from equations $(79)$ and (81) respectively as a function of $x$. First, notice that it is possible to bound the $S$-matrix (64) by

$$
\frac{1}{3} \hat{S}_{\omega_{p} \omega_{1} \omega_{2} \omega_{3} \omega} \leq S_{\omega_{p} \omega_{1} \omega_{2} \omega_{3}} \leq \hat{S}_{\omega_{p} \omega_{1} \omega_{2} \omega_{3}}
$$

where the modified $S$-matrix

$$
\hat{S}_{\omega_{p} \omega_{1} \omega_{2} \omega_{3}}=\frac{1}{3 \omega_{p} \omega_{1} \omega_{2} \omega_{3}}\left[I_{\omega_{p} \omega_{1} ; \omega_{2} \omega_{3}}+I_{\omega_{p} \omega_{2} ; \omega_{1} \omega_{3}}+I_{\omega_{p} \omega_{3} ; \omega_{2} \omega_{1}}\right]
$$

and $I_{\omega_{p} \omega_{1} ; \omega_{2} \omega_{3}}$ reads

$$
I_{\omega_{p} \omega_{1} ; \omega_{2} \omega_{3}}=\frac{\pi}{2^{9}} \int_{u_{\min }}^{u_{\max }} \frac{1}{u^{4}}\left[\left(\left(\sqrt{\omega_{p}}+\sqrt{\omega_{1}}\right)^{2}-u\right)\left(u-\left(\sqrt{\omega_{p}}-\sqrt{\omega_{1}}\right)^{2}\right)\right]^{3 / 2}\left[\left(\left(\sqrt{\omega_{2}}+\sqrt{\omega_{3}}\right)^{2}-u\right)\left(u-\left(\sqrt{\omega_{2}}-\sqrt{\omega_{3}}\right)^{2}\right)\right]^{3 / 2} d u .
$$




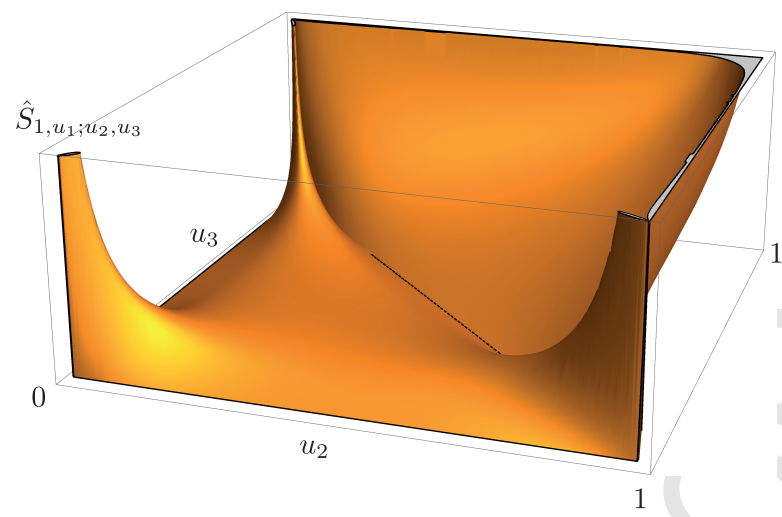

Figure 3: The bound $S$-matrix $\hat{S}_{\omega_{p} \omega_{1} \omega_{2} \omega_{3}}$ as a function of the dimensionless variables $u_{2}=\omega_{2} / \omega_{p}, u_{3}=\omega_{3} / \omega_{p}$, and here it is understood that $u_{1}=\omega_{1} / \omega_{p}$ and $u_{1}=u_{2}+u_{3}-1$ if $u_{2}+u_{3} \geq 1, u_{1}=1-\left(u_{2}+u_{3}\right)$ if $u_{2}+u_{3}<1$, and, finally, $u_{1}=0$ if $u_{2}+u_{3}=1$. Notice that $\hat{S}_{\omega_{p} \omega_{1} \omega_{2} \omega_{3}=0 \text { at }}$ the line $\omega_{2}+\omega_{3}=\omega_{p}$.

The integration limits are given by $u_{\min }=\max \left\{\left(\sqrt{\omega_{p}}-\sqrt{\omega_{1}}\right)^{2},\left(\sqrt{\omega_{2}}-\sqrt{\omega_{3}}\right)^{2}\right\}$ and $u_{\max }=\min \left\{\left(\sqrt{\omega_{p}}+\sqrt{\omega_{1}}\right)^{2},\left(\sqrt{\omega_{2}}+\sqrt{\omega_{3}}\right)^{2}\right\}$ (see Appendix 10.7 for more details). A surface plot of the $\hat{S}$-matrix is shown in Fig. 3. As it can be seen, $\hat{S}$ presents nodal lines at $\omega_{2}+\omega_{3}=\omega_{p}, \omega_{2}=0$ and $\omega_{3}=0$. Further, it presents four singular points at: $\omega_{2}=0 \& \omega_{3}=0, \omega_{2}=0 \& \omega_{3}=\omega_{p}$, $\omega_{2}=\omega_{p} \& \omega_{3}=0$, and $\omega_{2}=\omega_{p} \& \omega_{3}=\omega_{p}$, and two singular lines: $\omega_{2}=\omega_{p}$ and $\omega_{3}=\omega_{p}$.

We shall define $\hat{I}_{2 \leftrightarrow 2}^{(1)}$ and $\hat{I}_{3 \leftrightarrow 1}^{(1)}$ as estimated collisional integral using the upper bound $\hat{S}_{1 u_{1} u_{2} u_{3}}$ instead of the true $S$-matrix in equations (79) and (81) respectively. The upper and lower bound for the $S$-matrix (82) leads to the inequalities

$$
\frac{1}{3}\left|\hat{I}_{2 \leftrightarrow 2}^{(1)}(x)\right| \leq\left|I_{2 \leftrightarrow 2}^{(1)}(x)\right| \leq\left|\hat{I}_{2 \leftrightarrow 2}^{(1)}(x)\right| \quad \text { and } \quad \frac{1}{3}\left|\hat{I}_{3 \leftrightarrow 1}^{(1)}(x)\right| \leq\left|I_{3 \leftrightarrow 1}^{(1)}(x)\right| \leq\left|\hat{I}_{3 \leftrightarrow 1}^{(1)}(x)\right| .
$$

The above bounds guarantees that the convergence criterion for $I_{2 \leftrightarrow 2}^{(1)}$ and $I_{3 \leftrightarrow 1}^{(1)}$ are the same as for $\hat{I}_{2 \leftrightarrow 2}^{(1)}$ and $\hat{I}_{3 \leftrightarrow 1}^{(1)}$ respectively. A rather cumbersome calculation analyzing each singular point and nodal lines of the $S$-matrix (see Appendix 10.8 for details) shows that $\hat{I}_{2 \leftrightarrow 2}^{(1)}$ converges for $\frac{1}{2}<x<\frac{7}{4}$ while $\hat{I}_{3 \leftrightarrow 1}^{(1)}$ converges for $\frac{1}{4}<x<\frac{7}{4}$. Therefore the interaction can be considered local for $\frac{1}{2}<x<\frac{7}{4}$.

We have computed numerically both collisional integrals $\hat{I}_{2 \leftrightarrow 2}^{(1)}(x)$ and $\hat{I}_{3 \leftrightarrow 1}^{(1)}(x)$ for some values of $x$. The final exact values of $I_{2 \leftrightarrow 2}^{(1)}(x)$ and $I_{3 \leftrightarrow 1}^{(1)}(x)$ are in between the intervals shown in Fig. 4 for both collisional integrals.

\subsection{Log correction of the $K Z$ spectrum for an elastic plate}

We shall see here first that energy flux associated to the $x=1$ Zakharov exponent is zero. Replacing the collisional terms (78) and (80) into the equation for the energy flux (75), one gets

$$
P=-\int_{0}^{\omega} K^{3} \omega_{p}^{-3 x+2}\left(I_{3 \leftrightarrow 1}^{(1)}(x)+I_{2 \leftrightarrow 2}^{(1)}(x)\right) d \omega_{p}=\frac{K^{3} \omega^{-3(x-1)}}{3(x-1)}\left(I_{3 \leftrightarrow 1}^{(1)}(x)+I_{2 \leftrightarrow 2}^{(1)}(x)\right) .
$$

This energy flux must be independent of $\omega$, so that one takes the limit $x \rightarrow 1$ of this expression, that is:

$$
\lim _{x \rightarrow 1} P=\frac{K^{3}}{3}\left(\left.\frac{\partial I_{3 \leftrightarrow 1}^{(1)}(x)}{\partial x}\right|_{x=1}+\left.\frac{\partial I_{2 \leftrightarrow 2}^{(1)}(x)}{\partial x}\right|_{x=1}\right)=0 .
$$

Usually, for $x \neq 1$, the r.h.s. of (85) is a finite non-zero quantity, which allows to express the constant $K$ in terms of the energy flux $P$. However in the present case $x=1$ and the derivatives with respect to $x$ of $I_{3 \leftrightarrow 1}^{(1)}(x)$ and $I_{2 \leftrightarrow 2}^{(1)}(x)(79,81)$ are both zero. Therefore, the energy flux vanishes for the stationary power law solution of the form $n_{\omega} \propto \omega^{-1}$, in agreement with the fact that such solution corresponds to the equilibrium Rayleigh-Jeans distribution. In conclusion, a stationary solution with a constant flux may exist but it cannot be written as a simple power law in $\omega$. 


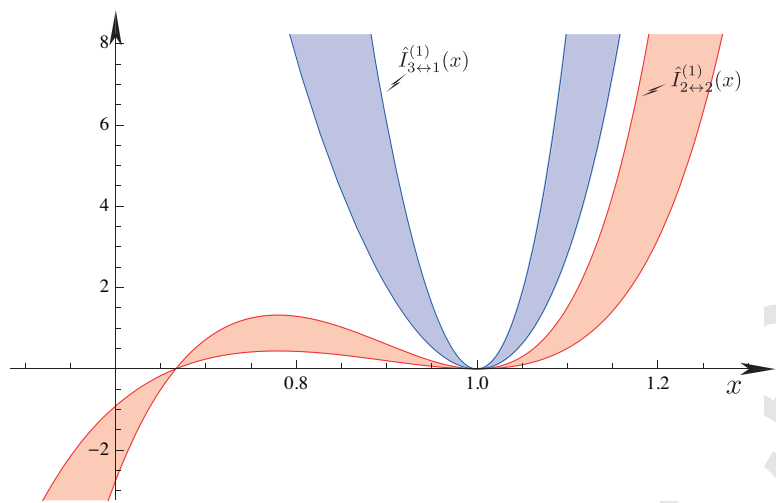

Figure 4: The bounds of the collisional integrals $\hat{I}_{2 \leftrightarrow 2}^{(1)}(x)$ and $\hat{I}_{3 \leftrightarrow 1}^{(1)}(x)$ as functions of $x$. The shadow domains represent the possible values of the exact collisional integrals $I_{2 \leftrightarrow 2}^{(1)}(x)$ and $I_{3 \leftrightarrow 1}^{(1)}(x)$.

This degeneracy of the Rayleigh-Jean distribution, which has been previously observed in plasma waves and non linear optics, suggests that the KZ spectrum is close to the thermodynamic equilibrium [3]. In the context of non linear optics (nonlinear Schördinger equation) the authors of [10] search for a logarithmic correction to the equilibrium solution, however the resulting constant energy flux solution was non local, hence meaningless. Further progress on the search for a Kolmogorov spectrum in optical turbulence has been achieved using an appropriate hidden parameter [11, 12]. For elastic plates we will follow a similar procedure as the one used in [10], we search for solution of the form

$$
n_{\omega}=K \omega^{-x} \log ^{z}\left(\omega / \omega_{c}\right) .
$$

To properly define the coefficient $K$, and the exponents $x$ and $z$, a third order perturbation expansion, in terms of the small parameter $\omega / \omega_{c} \ll 1$, should be carried out. In order to calculate the coefficient $K$ in terms of the energy flux, one needs to take carefully the limits, because the two limits $z \rightarrow 1 / 3$ and $x \rightarrow 1$ have to be taken independently (see details in Appendix 10.9). Cumbersome calculations lead to the constant energy spectrum

$$
n_{\omega}=\left(\frac{18 P}{I^{(1)^{\prime \prime}}(1)}\right)^{1 / 3} \frac{1}{\omega} \log ^{1 / 3}\left(\omega_{c} / \omega\right)
$$

which gives a positive wave action for $\omega<\omega_{c}$. Here $I^{(1)^{\prime \prime}}(1)=I^{(1)}{ }_{3 \leftrightarrow 1}{ }^{\prime \prime}(1)+I_{2 \leftrightarrow 2}^{(1)}{ }^{\prime \prime}(1)$ is the second derivative of the total collision integral where

$$
\left.I_{3 \leftrightarrow 1}^{(1)}{ }_{3 \leftrightarrow}^{\prime \prime}(1) \equiv \frac{\partial^{2} I_{3 \leftrightarrow 1}^{(1)}(x)}{\partial x^{2}}\right|_{x=1}=72 \pi \int_{O} S_{1 u_{1} u_{2} u_{3}}\left(u_{1} u_{2} u_{3}\right)^{-1}\left(u_{1} \log u_{1}+u_{2} \log u_{2}+u_{3} \log u_{3}\right)^{2} \delta\left(1-u_{1}-u_{2}-u_{3}\right) d u_{123}>0
$$

and

$$
\left.I_{2 \leftrightarrow 2}^{(1)}{ }^{\prime \prime}(1) \equiv \frac{\partial^{2} I_{2 \leftrightarrow 2}^{(1)}(x)}{\partial x^{2}}\right|_{x=1}=216 \pi \int_{I} S_{1 u_{1} u_{2} u_{3}}\left(u_{1} u_{2} u_{3}\right)^{-1}\left(-u_{1} \log u_{1}+u_{2} \log u_{2}+u_{3} \log u_{3}\right)^{2} \delta\left(1+u_{1}-u_{2}-u_{3}\right) d u_{123}>0 .
$$

The spectrum (87) show two main difference with respect to the result obtained in [10] for optical turbulence. In first place the pre factor is different since the first three order term in the expansion contribute to the flux while two of them where mistakenly neglected in [10]. Secondly an more important, the interaction are local for (87), hence is a meaningful turbulent cascade. The second derivative $I^{(1)^{\prime \prime}}(1)$ can be estimated as we have done for the collisional integrals in previous section since

$$
\frac{1}{3}\left|\hat{I}_{2 \leftrightarrow 2}^{(1)}{ }^{\prime \prime}(1)\right| \leq\left|I_{2 \leftrightarrow 2}^{(1)}{ }^{\prime \prime}(1)\right| \leq\left|\hat{I}_{2 \leftrightarrow 2}^{(1)}{ }^{\prime \prime}(1)\right| \quad \text { and } \quad \frac{1}{3}\left|\hat{I}_{3 \leftrightarrow 1}^{(1)}{ }^{\prime \prime}(1)\right| \leq\left|I_{3 \leftrightarrow 1}^{(1)}{ }^{\prime \prime}(1)\right| \leq\left|\hat{I}_{3 \leftrightarrow 1}^{(1)}{ }^{\prime \prime}(1)\right| \quad .
$$


Numerically we have

$$
\hat{I}_{2 \leftrightarrow 2}^{(1)}{ }^{\prime \prime}(1) \approx 0.29 \times 216 \pi \approx 197 \quad \& \quad \hat{I}_{3 \leftrightarrow 1}^{(1)}{ }^{\prime \prime}(1) \approx 6.12 \times 72 \pi \approx 1384 .
$$

Therefore, the coefficient $I^{(1)^{\prime \prime}}(1)$ is bounded by $527 \leq I^{(1)^{\prime \prime}}(1) \leq 1581$, hence the final numerical coefficient in the spectrum (87), namely $\left(18 / I^{(1)^{\prime \prime}}(1)\right)^{1 / 3}$, is bounded by 0.22 and 0.33 .

Remarkably, a similar procedure can be done for the inverse case $\omega / \omega_{c} \gg 1$, where the result is given by

$$
n_{\omega}=\left(\frac{18 P}{I^{(1)^{\prime \prime}}(1)}\right)^{1 / 3} \omega^{-1} \log ^{1 / 3}\left(\omega / \omega_{c}\right)
$$

which also gives a positive wave action for $\omega>\omega_{c}$. In both cases, this mathematical solution exhibits a cut-off frequency above (below) which the KZ spectra is null! The physical meaning of this cut-off frequency $\omega_{c}$ is not completely clear, for instance, could be associated to the existence of a warm cascade. Rewriting (87) as $n_{\omega}=\frac{1}{\omega}\left(T-\frac{18 P}{I^{(1)^{\prime \prime}}(1)} \log \omega\right)^{1 / 3}$ one can relate a "temperature" $T$ with the cut-off frequency scale $\omega_{c}$. Anyway, the logarithmic correction remain valid independently of the cut-off frequency scale.

The log-correction (87) to the KZ spectrum gives a correction to the frequency, estimated by the frequency shift formula (21):

$$
\omega_{2}(\boldsymbol{p})=\frac{9 \pi\left(1-\sigma^{2}\right)}{2 \rho h^{4}}\left(\frac{18 P}{I^{(1)^{\prime \prime}}(1)}\right)^{1 / 3}\left[\frac{\omega_{p}}{2 \omega_{c}} \int_{0}^{\log \left(\omega_{c} / \omega_{p}\right)} t^{1 / 3} e^{t} d t+\frac{\omega_{c}}{2 \omega_{p}} \int_{\log \left(\omega_{c} / \omega_{p}\right)}^{\infty} t^{1 / 3} e^{-t} d t\right],
$$

which is a function of $\omega_{p}$.

It is now important to investigate whether (and how) such KZ spectrum establishes in practice for elastic pates. In general, the experimental realization of wave turbulence cascade has always been a challenge due to different experimental limitations: for instance, the separation of scales between the injection and the dissipation, defining the so-called transparency window, is often difficult to achieve. Furthermore, realistic dissipation might not be concentrated only at small scale, perturbing the cascade process and sometime the measurement is not adapted to the quantities involved in the WTT. It can be thus interesting to investigate first the KZ spectra using numerical simulations of the dynamics, where the injection and the dissipation can be imposed in order to satisfy the WTT hypothesis.

\section{Numerical validations}

In this section, we investigate numerically the different regimes studied theoretically above. We address in details the vibrations of an elastic plate in two idealized situations: firstly, we study the dissipation free (Hamiltonian) dynamics, to observe the dynamics towards the Rayleigh-Jeans equilibrium. Then, injecting energy at large scales and adding dissipation at small scales only, we mimic the ideal situation of direct cascade in wave turbulence.

Solving numerically the coupled set of dynamical equations for the plates can be done thanks to a pseudo-spectral method which take advantage of the linear wave dynamics in the Fourier space. Although spectral methods can allow different boundary conditions, it is usually written for periodic boundary conditions as it will be the case further on. In fact, periodic boundary conditions provide the natural framework to investigate wave turbulence. On the other hand, methods using finite difference scheme would be more appropriate to account for general boundary conditions although it might be less accurate to capture the wave dynamics $[32,52]$. As described below the pseudo-spectral method uses the real space for computing the non-linear term while the integration is performed in the Fourier space thanks to an Adams-Bashford scheme.

\subsection{Numerical Scheme}

We present the numerical scheme for the elastic plate equations $(1,2)$ since this general framework can be adapted easily to other situations. The set of equations (1) and (2) reads in the Fourier space:

$$
\begin{aligned}
\ddot{\zeta}_{\boldsymbol{k}} & =-\frac{k^{4}}{4} \zeta_{\boldsymbol{k}}+N L_{\boldsymbol{k}}^{(1)} ; \\
k^{4} \chi_{\boldsymbol{k}} & =N L_{\boldsymbol{k}}^{(2)} .
\end{aligned}
$$


The functions $N L_{\boldsymbol{k}}^{(1)}$ and $N L_{\boldsymbol{k}}^{(2)}$ are the Fourier transform of the nonlinear terms $N L^{(1)}(\boldsymbol{x}, t)=\{\zeta, \chi\}$ and $N L^{(2)}(\boldsymbol{x}, t)=$ $-\frac{1}{2}\{\zeta, \zeta\}$ respectively. The dynamics of the plate, eq. (91), can be rewritten as:

$$
\frac{d X_{k}}{d t}=\mathcal{L}_{k} \cdot X_{k}+B_{k}
$$

with,

$$
X_{\boldsymbol{k}}=\left[\begin{array}{c}
\zeta_{\boldsymbol{k}} \\
\dot{\zeta}_{\boldsymbol{k}}
\end{array}\right], \quad B_{\boldsymbol{k}}=\left[\begin{array}{c}
0 \\
N L_{\boldsymbol{k}}^{(1)}
\end{array}\right] \text { and } \mathcal{L}_{k}=\left[\begin{array}{cc}
0 & 1 \\
-\omega_{k}^{2} & 0
\end{array}\right]
$$

While, the second equation (92) makes a simple relation in the Fourier space for $\chi_{\boldsymbol{k}}$ :

$$
\chi_{k}=\frac{1}{k^{4}} N L_{\boldsymbol{k}}^{(2)} .
$$

Integrating (93) between $t$ and $t+d t$ leads to:

$$
X_{\boldsymbol{k}}(t+d t)=e^{d t \mathcal{L}_{k}} X_{\boldsymbol{k}}(t)+\int_{0}^{d t} e^{(d t-s) \mathcal{L}_{k}} B_{\boldsymbol{k}}(t+s) d s .
$$

Notice that the exponential of the $\mathcal{L}_{k}$ matrix is very simple since $\mathcal{L}_{k}^{2}=-\omega_{k}^{2} \mathrm{Id}$, where Id is the $2 \times 2$ identity matrix. Therefore, the numerical scheme follows the general algorithm:

1. compute in the real space $N L^{(1)}(\boldsymbol{x}, t)$

2. calculate $N L_{\boldsymbol{k}}^{(1)}(t)$ using the Fourier transform

3. in the Fourier space, integrate equation (94) using a polynomial expansion of $B_{k}(t+s)$, giving $\zeta_{\boldsymbol{k}}(t+d t)$ and $\dot{\zeta}_{\boldsymbol{k}}(t+d t)$.

4. compute $\zeta(\boldsymbol{x}, t+d t)$ and its derivatives by inverse Fourier transform for computing $N L^{(2)}(\boldsymbol{x}, t+d t)$

5. use the Fourier transform to calculate $N L_{\boldsymbol{k}}^{(2)}(t+d t)$

6. compute $\chi_{\boldsymbol{k}}(t+d t)$ and make its inverse Fourier transform

The poynomial expansion for $B_{\boldsymbol{k}}(t+s)$ is performed using the known values of $B_{\boldsymbol{k}}(t), B_{\boldsymbol{k}}(t-d t), B_{\boldsymbol{k}}(t-2 d t) \ldots$ depending on the order of the polynomial expansion needed (order one polynomial is used in our numerics).

The plate is discretized in the real space using a constant grid size $d x$, so that the discrete set of computation points is $\left(x_{i}, y_{j}\right)=(i \cdot \Delta x, j \cdot \Delta x)$. The computational domain is defined by the number, $N$, of grid points in each direction, leading to a square plate of size $L \times L=(N \cdot \Delta x) \times(N \cdot \Delta x)$, and taking $0 \leq i, j<N$. The Fourier transform of the discretized field $\hat{\zeta}_{k}$ in $(91,92)$ is defined using the fftw library [53]:

$$
\hat{\zeta}_{\boldsymbol{k}}=\frac{1}{N^{2}} \sum_{i, j=0}^{N} \zeta\left(x_{i}, y_{j}\right) e^{-i\left(k_{m} \cdot x_{i}+k_{n} \cdot x_{j}\right)},
$$

for wave-numbers

$$
\boldsymbol{k}=\left(k_{m}, k_{n}\right)=\left(\frac{2 \pi m}{L}, \frac{2 \pi m}{L}\right) \text { for }-\frac{N}{2}<m, n \leq \frac{N}{2} .
$$

Notice that a rescaling factor is introduced between the discretized field $\hat{\zeta}_{\boldsymbol{k}}$ in the numerics and the continuous one $\zeta_{\boldsymbol{k}}$. Using the discretization of the integral term:

$$
\zeta_{\boldsymbol{k}}(t)=\frac{1}{2 \pi} \int \zeta(\boldsymbol{r}, t) e^{-i \boldsymbol{k} \cdot \boldsymbol{r}} d \boldsymbol{r}=\frac{1}{2 \pi} \sum_{i, j=0}^{N} \zeta\left(x_{i}, y_{j}\right) e^{-i\left(k_{m} \cdot x_{i}+k_{n} \cdot x_{j}\right)} \Delta x^{2}=\frac{L^{2}}{2 \pi} \hat{\zeta}_{\boldsymbol{k}}(t) .
$$

Similarly, the $\delta^{(2)}(\boldsymbol{k})$ factor involved in the relation (19) between $\left|\zeta_{\boldsymbol{k}}\right|^{2}$ and $n(\boldsymbol{k})$ induces a $1 / \Delta k^{2}=(L /(2 \pi))^{2}$ factor in the discretized relation:

$$
\left\langle\left|\hat{\zeta}_{k}\right|^{2}\right\rangle=\frac{1}{L^{2}} \frac{n_{k}}{\omega_{k}}
$$

Finally, dealiaising has been tested for the Fourier transform with no significant changes so that we usually do not use it in order to maximize the Fourier range of our simulations.

Now that we have the general scheme to solve numerically the plate dynamics, wave action and/or energy injection as well as dissipation can be easily implemented as described below. 


\subsection{Decaying turbulence of an isolated system: towards the Thermodynamical Equilibrium}

Prior to observe KZ spectra induced by injection/dissipation balance, it is interesting to observe for a free system how the dynamics converges to the thermodynamical equilibrium. Additionally, it will provide a validation of the numerical method and an estimation of its accuracy. In the case of the elastic plate, the Rayleigh-Jeans equilibrium spectrum $\left(n_{k}=T / \omega_{k}\right)$ gives for the Fourier transform of the deflection:

$$
\left\langle\left|\zeta_{k}\right|^{2}\right\rangle=\frac{n_{k}}{\omega_{k}} \propto \frac{T}{k^{4}}
$$

In that purpose, we start with a Gaussian distribution of the Fourier modes as initial condition

$$
\zeta_{\boldsymbol{k}}(t=0)=A e^{-\frac{\sigma_{0}^{2} k^{2}}{2}+i \theta_{\boldsymbol{k}}}
$$

where $\sigma_{0}$ is the width of the distribution and $\theta_{\boldsymbol{k}}$ is a random phase with $\theta_{-\boldsymbol{k}}=-\theta_{\boldsymbol{k}}$ since $\zeta$ is a real field. Starting with such initial conditions we observe the formation of the Rayleigh-Jeans spectrum as shown on figure 5 -a) for a typical case. There the relaxation towards a Rayleigh-Jeans spectrum is seen although for the latest time reached by the numerics, only intermediate scales are close to this regime while small and large scale modes are still far from equilibrium.

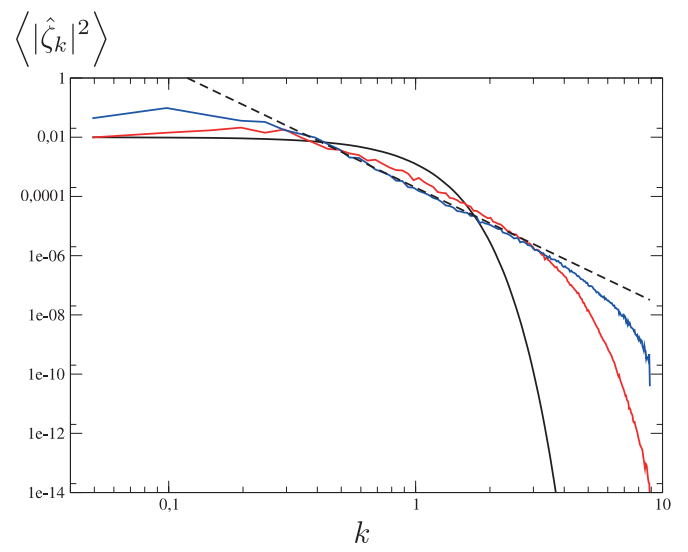

a)

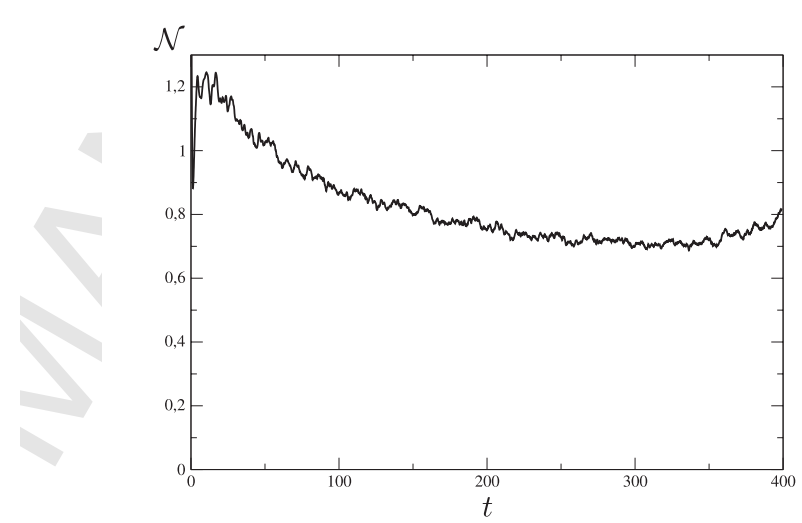

b)

Figure 5: a) The density spectrum $\left\langle\left|\hat{\zeta}_{k}\right|^{2}\right\rangle$ at different times for the free dynamics (no injection nor dissipation), starting with a Gaussian spectrum at $t=0$ (black curve). The spectra for $t=100$ (red curve) and $t=300$ (blue curve) unit times are shown while the expected $k^{-4}$ law is plotted (dashed line) for comparison. The spectrum is obtained by computing the mean value of $\left|\hat{\zeta}_{k}\right|^{2}$ through an angular average. The simulation was performed on a $128 \times 128$ plate with $d x=0.5$ in dimensionless units (so that the simulation is done with $256 \times 256$ modes. b) The evolution of the wave action $\int n_{k} d k$ with time during the relaxation of the dynamics towards the statistical equilibrium.

Ironically, such numerical simulations are the hardest to perform for two main reasons: first, the relaxation is much slower than when energy is injected at one scale and dissipated at some scales, as we will show below. Moreover, as the predicted spectrum illustrate it, the equilibrium spectrum would correspond to very high amplitude of low $k$ modes and the numerics becomes there no more stable, saying nothing about nonlinear effects due to these high amplitudes. For such numerical simulation, the energy was conserved up to $0.5 \%$ while the wave action decreases with time as plotted on figure 5 -b).

In fact, one can observe that the wave action seems to slightly increase for the later times reached by the numerics $(t>350)$. This could be related to the high amplitudes reached by the low- $k$ modes that increase the wave action for constant energy. However, at the same time, the numerics becomes unstable, precisely because the low- $k$ modes have high amplitudes and the energy is no more preserved by the scheme. We have thus not been able to determine whether this increase was corresponding to a physical effects (the filling of the low- $k$ modes) or whether it was a numerical artifact. Finally, let us notice that for low- $k$, because of the high amplitude predicted by the theory, the equipartition spectrum should deviate from the $k^{-4}$ law which is obtained in a weak amplitude limit and nonlinear terms should be accounted for determining the spectrum (see for instance [51] for a different case where a soliton-like solution is valid at low- $k$ ). 
a)

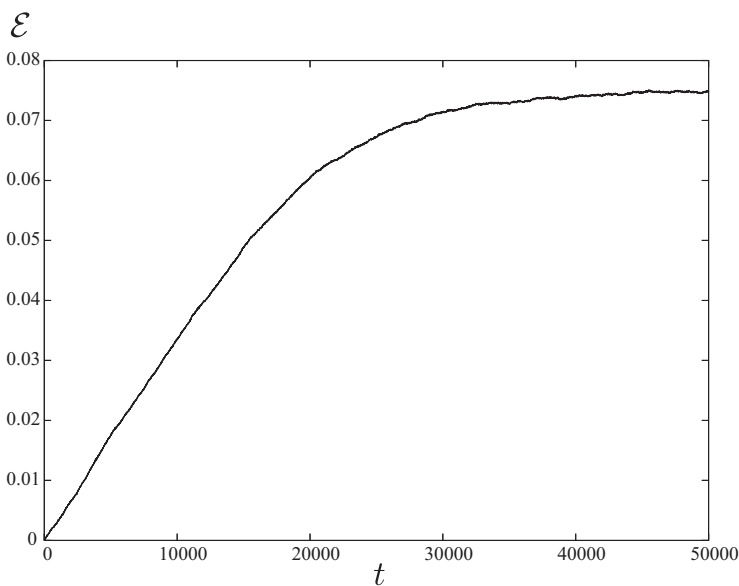

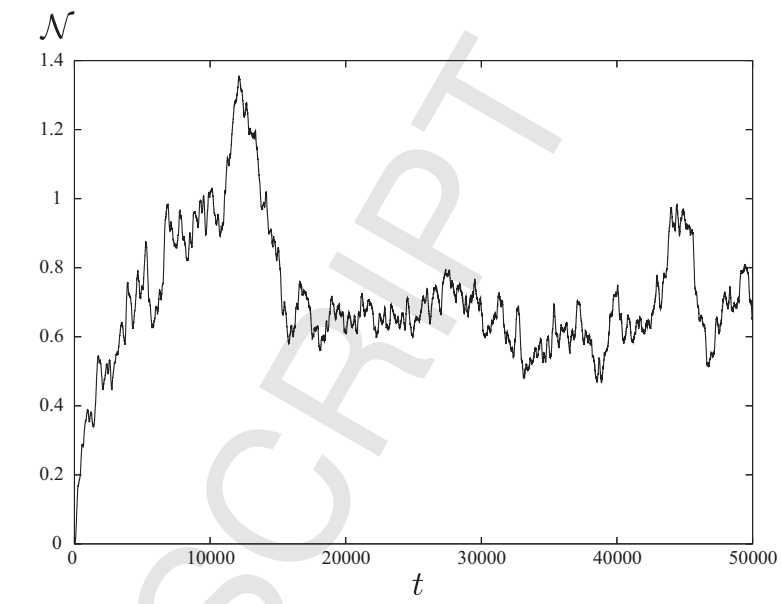

b)

Figure 6: Energy (a) and wave action (b) as function of time for a vibrating plate of size $L \times L=512 \times 512$ over $1024 \times 1024$ Fourier modes. The injection at large scale and dissipation at small scale is performed using $k_{i}=0.1, A_{i}=1.25 \cdot 10^{-4}$ and $k_{d}=5.5, \eta=0.5$.

\subsection{Kolmogorov spectra of an Energy cascade}

Our numerical scheme provides an ideal tool to investigate the wave turbulence processes: indeed, it is easy in Fourier space to impose numerically injection and dissipation at prescribed scales, i.e. for limited values in $k$. Formally, injecting energy (and wave action since it is coupled) and dissipation can be implemented in the dynamical equation (12) following

$$
\frac{\partial^{2} \zeta}{\partial t^{2}}=-\frac{1}{4} \Delta^{2} \zeta+\{\zeta, \chi\}+\mathcal{I}+\mathcal{D}
$$

where $\mathcal{I}$ and $\mathcal{D}$ stand for the injection and dissipation processes respectively which will be described in the Fourier space (noted $I_{\boldsymbol{k}}$ and $D_{\boldsymbol{k}}$ ). To mimic the configuration potentially leading to a direct cascade, we take for instance

$$
I_{\boldsymbol{k}}=A_{i} \frac{k^{2}\left(4 k_{i}^{2}-k^{2}\right)}{k_{i}^{4}} \eta_{\boldsymbol{k}} \text { for }|k| \leq 2 k_{i}, \text { and } I_{\boldsymbol{k}}=0 \text { otherwise. }
$$

Here, the injection amplitude is centered around the typical wave number $k_{i}$ with a random noise delta correlated in time (following $\eta_{\boldsymbol{k}}^{*}=\eta_{-\boldsymbol{k}}$ ). Regardless the physical dissipation mechanisms present in plate dynamics [26, 27], we will first introduce a phenomenological dissipation at small scale in order to investigate the cascade process itself:

$$
D_{k}=-\eta\left(k^{2}-k_{d}^{2}\right) \dot{\zeta}_{k} \text { for }|k| \geq k_{d} \text {, and } D_{k}=0 \text { otherwise. }
$$

The dissipation is thus only present for scales smaller than the cut-off scale defined by the wave-number $k_{d}$. Starting with a still plate, a stationary regime is reached after a long transient (few thousands of unit time), as shown on figure (6) where the time evolution of the energy and the wave action are plotted.

In this statistically steady state, the deformation spectrum $\left\langle\left|\zeta_{\boldsymbol{k}}\right|^{2}\right\rangle$ exhibits a power-like behavior in good agreement with the predicted spectrum:

$$
\left\langle\left|\zeta_{\boldsymbol{k}}\right|^{2}\right\rangle=\frac{n(k)}{\omega_{k}} \propto P^{1 / 3} \frac{\log ^{1 / 3}\left(\frac{k_{d}}{k}\right)}{k^{4}}
$$

as shown on Fig. 7-a. Notice that the cut-off wave-number $k_{c}$ of equation (87) is exactly that of the dissipation scale $k_{d}$ ! In the figure, we observe that the logarithmic correction term is pertinent near the cut-off where it describes very precisely the spectrum. Nevertheless, let us emphasize that the mathematical cut-off of the KZ solution is not seen numerically: actually for $k>k_{c}$ the spectrum is not exactly zero indicating that higher order terms (remember that the KZ solution is obtained by an expansion of the solution) are present. This result is not surprising in fact, since the derivation of the logarithmic correction is obtained through a perturbative approach to the Rayleigh-Jeans spectrum. Therefore, it cannot be valid near the cutoff where 
a)

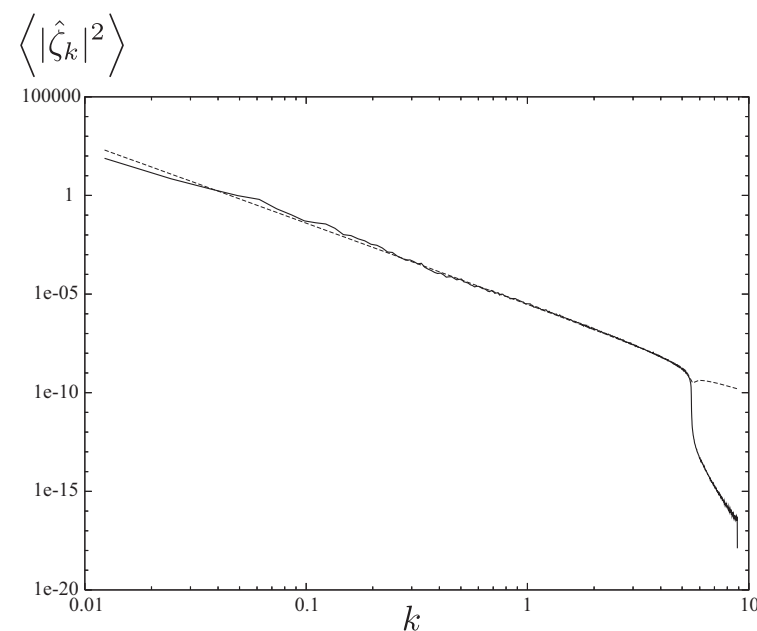

$P(t) / P_{s t}$

c)

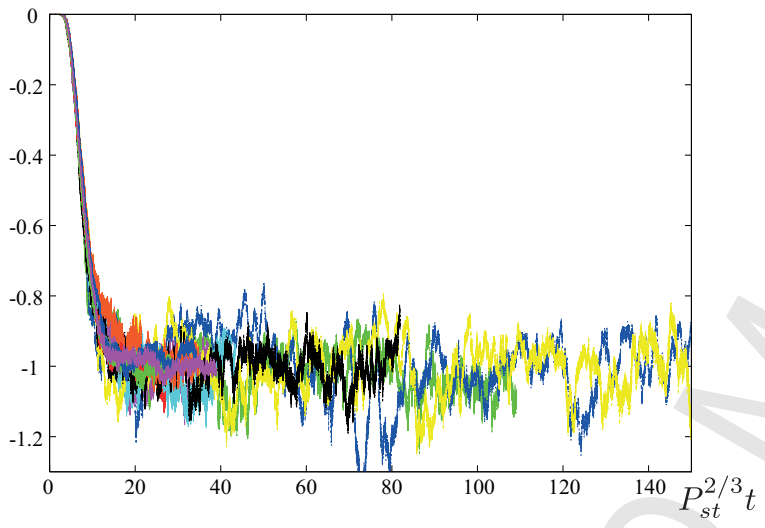

b)

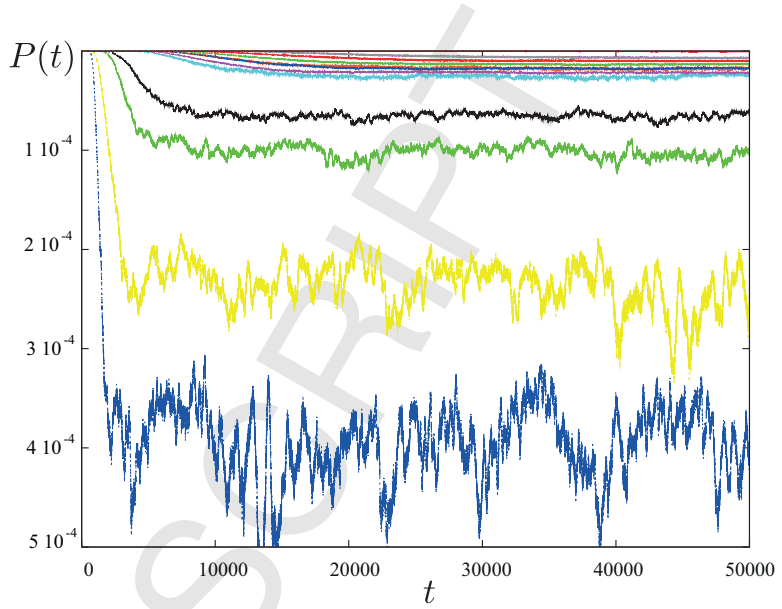

$\hat{C}(k)$

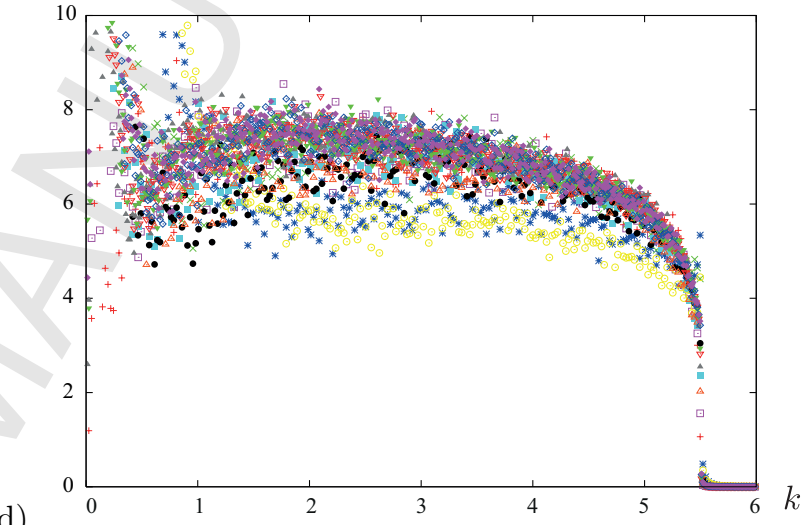

Figure 7: a) Numerical power spectrum (solid line) of the displacement field $\left\langle\left|\hat{\zeta}_{\boldsymbol{k}}\right|^{2}\right\rangle$ as a function the wave number $k$ for the same situation than figure (6). The dashed line shows the expected relation (96) deduced from the weak turbulence theory with the prefactor $2.5 \times 10^{-6}$ and using exactly for the cut-off wave-number $k_{c}=k_{d}=5.5$. b) Dissipation rate density (97) as a function of time for the vibrating plate described above for varying injection amplitudes $A_{i}=1.25 \times 10^{-4}, 1.5 \times 10^{-4}, 1.75 \times 10^{-4}, 2 \times 10^{-4}$ and $2.25 \times 10^{-4}$. Simulations for a half-size smaller plate $L \times L=256 \times 256$ with $512 \times 512$ modes are also shown for varying injection amplitudes $A_{i}=1.25 \times 10^{-4}, 2.5 \times 10^{-4}, 4 \times 10^{-4}, 5 \times 10^{-4}, 8 \times 10^{-4}, 0.001,0.0015$ and 0.002. After a short transient depending on the injection rate, the dissipation rate converges towards a stationary value $P_{s t}$ (plus fluctuations) that we identify with the energy flux of the cascade. The dissipation rates then exhibit small fluctuations around $P_{s t}$. c) The same dissipation rate curves (for all the 13 configurations) rescaled by the stationary dissipation rate $P_{s t}$ while the time is rescaled by $P_{s t}^{2 / 3} t$, showing that the time scale of the transient regime is precisely $P_{s t}^{-2 / 3}$ as it can be deduced from the kinetic equation eq. (20). d) The spectra for all the injection amplitudes of figures b) and c) (thus for different system lengths $L=512$ and $L=256$ ) rescaled by the expected energy flux dependence $\hat{C}(k)$ defined through (99) vs. the wavenumber $k$, showing an almost single universal spectrum curve.

the logarithmic term becomes dominant. Furthermore, near this cut-off, the kinetic equation becomes itself questionable since the dissipation time scale dominates the nonlinear one involved in the WWT.

The statistically state of the dynamics can also be illustrated with the energy dissipation rate which reaches a stationary regime for large time, as shown on Fig. 7-b for different injection amplitudes and system sizes $(L=512$ and $L=256$, for the same discretization mesh $d x=0.5)$. This dissipation rate density is in fact easily computed from the plate dynamics $(95)$ and reads:

$$
P(t)=-\eta \int_{k_{d}}^{k_{c}}\left(k^{2}-k_{d}^{2}\right)\left|\dot{\zeta}_{k}\right|^{2} d \boldsymbol{k} .
$$


Fig. 7-b shows that the dissipation rate decreases during a transient dynamics until reaching a stationary state plus fluctuations, where the energy injected at large scale is statistically balanced by the dissipation. In this stationary regime, we can easily extract the mean value $P_{s t}$ of the dissipation rate that is identified thus with the energy flux density of the energy cascade. The dependence of transient dynamics with $P_{s t}$ can be inferred from the kinetic equation (20). Indeed, introducing the $P_{s t}^{1 / 3}$ factor of the KZ spectra in the wave number densities $n(\boldsymbol{k})$, it leads to a $P_{s t}^{-2 / 3}$ time scale for the unsteady dynamics. This can be observed on Fig. 7-c where the dissipation rate (rescaled by the steady mean value $P_{s t}$ ) is plotted as function of $P_{s t}^{2 / 3} t$ for all the injection rates and system sizes investigated here. The different curves collapse into a single one, proving the $P_{s t}^{-2 / 3}$ time scale involved in the transient dynamics. Furthermore, it is tempting to check the $P_{s t}^{1 / 3}$ prefactor in the cascade spectrum itself. In that purpose, we need to compute precisely the prefactor involved for the displacement field Fourier transform by the numerical discretization, due to the contribution $L^{2} /(2 \pi)$ in the Fourier transform and that $(L /(2 \pi))^{2}$ of the Dirac function in the $n(\boldsymbol{k})$ definition, yielding the following form for the numerical spectrum:

$$
\left\langle\left|\hat{\zeta}_{\boldsymbol{k}}\right|^{2}\right\rangle=\frac{n_{k}}{\omega_{k} L^{2}}=\frac{2^{7 / 3} C}{L^{2}} P_{s t}^{1 / 3} \frac{\ln ^{1 / 3}\left(\kappa^{*} / k\right)}{k^{4}} .
$$

The different spectra are now rescaled according to the formula (98) above, plotting

$$
\hat{C}(k)=\frac{L^{2} k^{4}\left\langle\left|\hat{\zeta}_{k}\right|^{2}\right\rangle}{2^{7 / 3} P_{s t}^{1 / 3} \ln ^{1 / 3}\left(\kappa^{*} / k\right)}
$$

as function of $k$ using the mean value $P_{s t}$ of the dissipation rate measured in the statistically stationary regime. In the cascade domain, this rescaling should give the constant $\hat{C}(k)$ computed using the weak turbulence theory. Indeed, the weak turbulence cascade manifests here as a plateau regime for the inertial range (typically here for $1<k<5.5$ between the injection and dissipative ranges), where all the spectra collapse into a single curve. Notice however that the plateau regime is not perfectly constant as would suggest the WTT. Furthermore, the spectra in this plateau regime leads to $\hat{C}(k) \approx 7 \pm 1$, while the constant $C$ of the WTT is expected to lie between 0.22 and 0.33 . We have not been able yet to determine the origin of such difference that we will be consider elsewhere.

\section{Selected Topics}

In this last section, we first discuss how experiments compare with the WTT and explain where the differences might most probably come from. Then recent results on high forcing, breakdown of wave turbulence and potential inverse cascades are also presented and, finally we draw different perspectives of this work, in particular on curved (cylindrical and spherical shell) shells, and on the $h \rightarrow 0$ asymptotic limit of strong plate turbulence.

\subsection{Experimental observations.}

Soon after our prediction of the WTT KZ spectra for elastic plates, two different experimental groups have measured independently the energy spectrum of a vibrating elastic plate $[21,22]$. By vibrating the plate at low frequency, both found a turbulent regime with a large inertial range but with noticeable differences with the predicted WTT energy spectra (23): firstly, instead of a flat spectrum (up to the logarithmic correction), they found a slight decrease in agreement with $\omega^{-0.5 \pm 0.1}$. Furthermore, they both observed a dependence of the spectrum amplitude more consistent with a $P^{1 / 2}$ law than the expected $P^{1 / 3}$. Different explanations have been proposed for these discrepancies: a lack of isotropy of the system due to the rectangular shape of the plate [23], three waves resonances due to natural curvature of the plate and/or finite size effects [21, 24] and the specific dissipation valid in plates [26]. In fact, while the two first reasons were not shown to have strong effects on the turbulent spectra, recent results have proven that the dissipation in elastic plates is the main reason for the differences between the WTT predictions and the experiments [27, 28]. Indeed, the damping in real plates appears to be present at all scales (although more important at small scales) perturbing thus the inertial range of the WTT and leading to the steeper spectra observed experimentally. Finally, the dependence of the spectra on the energy flux $P$ appears also affected by this real damping, although the $P^{1 / 2}$ was not obtained when simulating the dynamics using the realistic dissipation. 


\subsection{Beyond wave turbulence: breaking wave turbulence, D-cones, ridges, high forcing and intermittencies}

As already discussed along the paper, it has been stressed that weak turbulence is not a completely satisfactory theory, because it always exhibits a breakdown at either large or small scales $[4,48,54]$. Because the theory is based on an asymptotic (ordered) perturbation procedure, its self-consistency requires higher-order terms to be smaller than lower-order terms, a property that should be verified for every scale, i.e. every wave number $k$. Naturally, such a self-consistency cannot be verified by the equilibrium solution (62), neither by the constant energy flux since they are essentially power-law solutions.

As discussed by Newell and collaborators [4, 48, 54], a well-ordered asymptotic expansion procedure is valid as long as the nonlinear frequency (only using scaling estimate here):

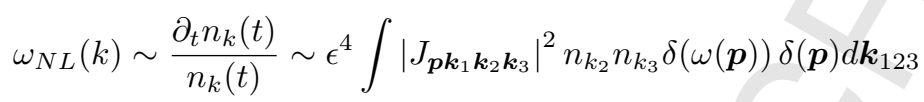

is much smaller than the linear frequency $\omega(k)$. Keeping in mind that for the elastic plate the $J$-matrix scales as $k^{0}$ and $\omega(k)=k^{2} / 2$, then one gets the condition

$$
\frac{\omega_{N L}(k)}{\omega(k)} \sim \epsilon^{4} n_{k}^{2} \ll 1
$$

The weak turbulence equations are thus valid for $\epsilon^{2} n_{k} \ll 1$ defining the crossover wavenumber $k_{N L}$ through $\epsilon^{2} n\left(k_{N L}\right) \sim 1$. For instance, for the KZ spectrum (22)

$$
\frac{k_{N L}^{2}}{\ln ^{1 / 3}\left(k_{N L} / \kappa^{*}\right)} \sim \epsilon^{2} P^{1 / 3}
$$

so that one expects breakdown of the WTT for $k<k_{N L}$, that is at large scale. The breakdown of the WTT arises therefore for plates at large scale primarily (something on which we will come back in Sec. 8.3. However, this crossover scale has to be compared to the other large scales involved in the system, particularly the injection scale $k_{i}$ and the size of the domain $L$ leading to a minimal wave number $k_{0}=2 \pi / L$ (in general, one has however $k_{i n}>k_{0}$.). Thus, for instance, if $k_{N L}$ is outside the frequency-bandwidth of interest delimited by the infrared, $k_{i r}=\min \left\{k_{i}, k_{0}\right\}$ and the ultraviolet cut-offs $k_{c}$, or in other words, if $\left(k_{N L} \notin\left[k_{i r}, k_{c}\right]\right)$, the weak turbulence description is safe. For a finite system size, one has always $k_{N L} \ll k_{i r}$ in the WTT asymptotic limit $\epsilon \rightarrow 0$ and the kinetic theory holds for any wavenumber $k$. Notice however that in that case some problems may come when the other asymptotic limit, the thermodynamic limit $L \rightarrow \infty$, is considered. However this is not the general situation and one should expect to observe the effect of the breakdown of WTT in the large scale of the KZ spectra.

Finite amplitude effects arise on the wave turbulence spectrum as the injection amplitude (and therefore the flux $P$ ) increases, as already observed in $[35,34,38]$, leading eventually to the breakdown of wave turbulence at large scales. Figure (8) shows how the spectrum is affected by higher injection amplitude: the theoretical wave turbulence behavior is changed as axpected mostly at large scale where the amplitudes of the modes become much higher than one. There, for the highest amplitude plotted, the slope of the deformation for the low- $k$ modes $\left(|\nabla \zeta|^{2} \sim k^{2}\left\langle\left|\zeta_{\boldsymbol{k}}\right|^{2}\right\rangle\right)$ is of order one, so that the weak amplitude hypothesis is no more valid. Similarly, one can show in this regime the breakdown of wave turbulence since the nonlinear frequency becomes bigger than the linear frequency of the waves [38] In fact, in this case, it has been shown [34] that the spectrum follows at large scale (small $k$ ) a scaling compatible with that of $d$-cones, leading to $\left|\zeta_{k}\right|^{2} \propto k^{-6}$, so that the vibrations of the plates can be interpreted as oscillations of ridges separating $d$-cones (see figure 9 for an illustration of the plate deformation). Finally, this high forcing regime corresponds also to the occurrence of intermittencies in the statistical properties of the turbulent fields [38].

\subsection{A possible inverse cascade?}

Although an inverse cascade of constant flux of wave action is not possible for vibrating plate because of the $3-1$ wave resonances that do not conserve the wave action, one can still question whether injecting deformation at small scale (large $k$ ) can give rise to an inverse transfer of some quantities (a priori wave action) towards large scales. In fact, in the kinetic equation, the absence of an inverse cascade solution is due to the presence of these collision terms that violate the wave-action conservation. As explained above, if these collision terms vanish, one would expect an inverse cascade spectrum with the following properties (see the end of Section 6.1):

$$
n(k) \propto k^{-4 / 3} \text { giving }\left\langle\left|\zeta_{k}\right|^{2}\right\rangle \propto k^{-10 / 3} .
$$

We can thus investigate such processes numerically by injecting at small $k$ following:

$$
I_{k}=A_{i} \frac{\left(k^{2}-\left(k_{i}-\delta_{i}\right)^{2}\right)\left(\left(k_{i}+\delta_{i}\right)^{2}-k^{2}\right)}{k_{i}^{4}} e^{i \theta_{k}} \text { for } k_{i}-\delta_{i} \leq|k| \leq k_{i}+\delta_{i}, \text { and } I_{k}=0 \text { otherwise. }
$$




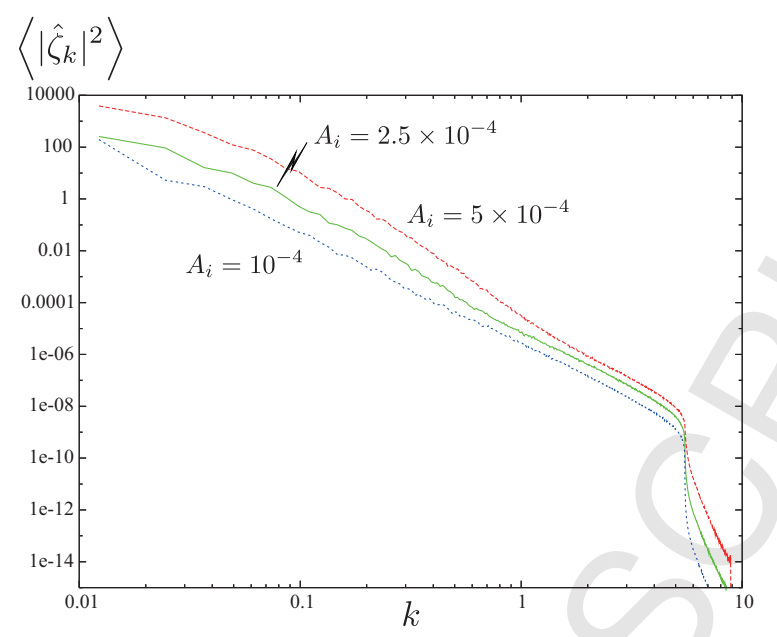

Figure 8: Displacement spectra as the injection amplitude increases with $A_{i}=1 \times 10^{-4}, 2.5 \times 10^{-4}$ and $5 \times 10^{-4}$ for increasing spectrum respectively.
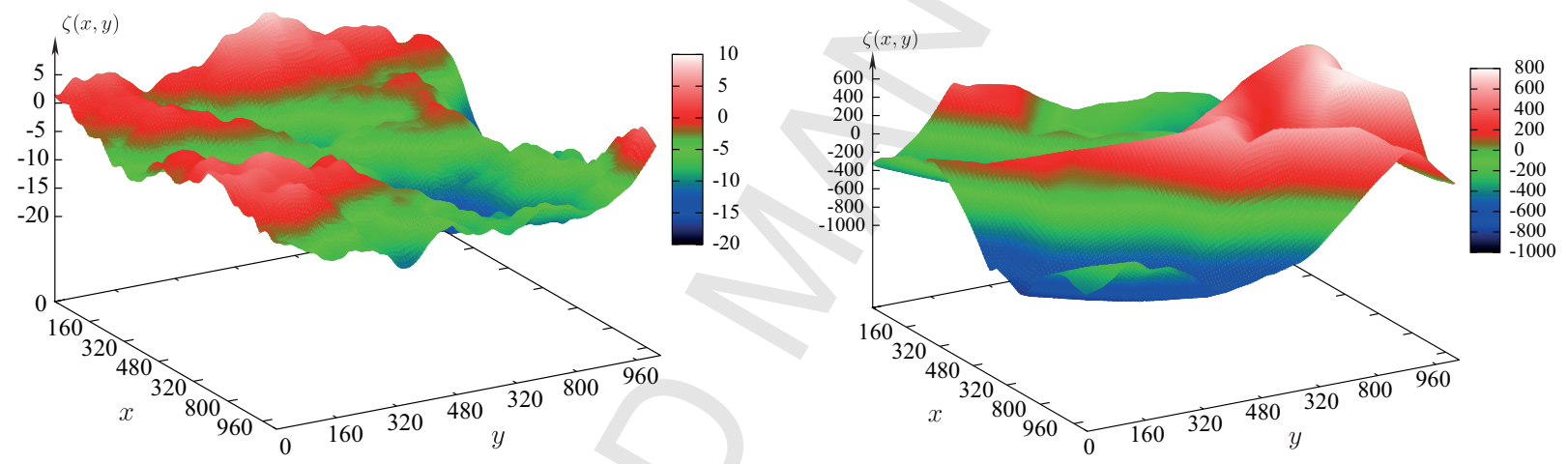

Figure 9: Plate deformation for two different injection amplitude a) low injection for which the KZ spectrum is valid; b) much larger injection, for which the breakdown of WTT is present. Notice the large amplitude of deformation and the presence of ridges. The simulations were done $L_{x}=L_{y}=1024$, the mesh size $d x=0.5$ and $h=0.5$.

with dissipation at small scales only:

$$
D_{k}=-\eta\left(k^{2}-k_{d}^{2}\right) \dot{\zeta}_{k} \text { for }|k| \geq k_{d}, \text { and } D_{k}=0 \text { otherwise. }
$$

Notice that this process injects both energy and wave action since one cannot separate them formally. However, although it is needed to dissipate at small scale the energy to reach a stationary regime, there is a priori no need to pump the wave action at large scale: it should be automatically done by the dynamics since the wave-action is not conserved. This configuration has been studied recently in great details in [30], showing a self-similar singular dynamics followed by a relaxation process that converges at large scale towards the expected inverse cascade spectrum eq. (102).

The existence of this inverse cascade process has a practical consequence on the spectrum for a general injection: one should observe a direct cascade towards the small scales and an inverse one towards the large scales. Again, this can be easily simulated within our numerical scheme by taking $k_{d}$ much larger than $k_{i}+\delta_{i}$, following the formalism described above. Figure 10 shows such spectrum for $k_{i}=0.6, \delta=0.2$ and $k_{d}=5.5$. Two behaviors are identified and compared with the theoretical predictions with very good agreement. The direct cascade law (96) is observed for $k_{i}+\delta<k<k_{d}$ (regardless the logarithmic correction) and the inverse cascade regime $\left\langle\left|\zeta_{k}\right|^{2}\right\rangle \propto k^{-10 / 3}$ is seen for $k<k_{i}-\delta$. 


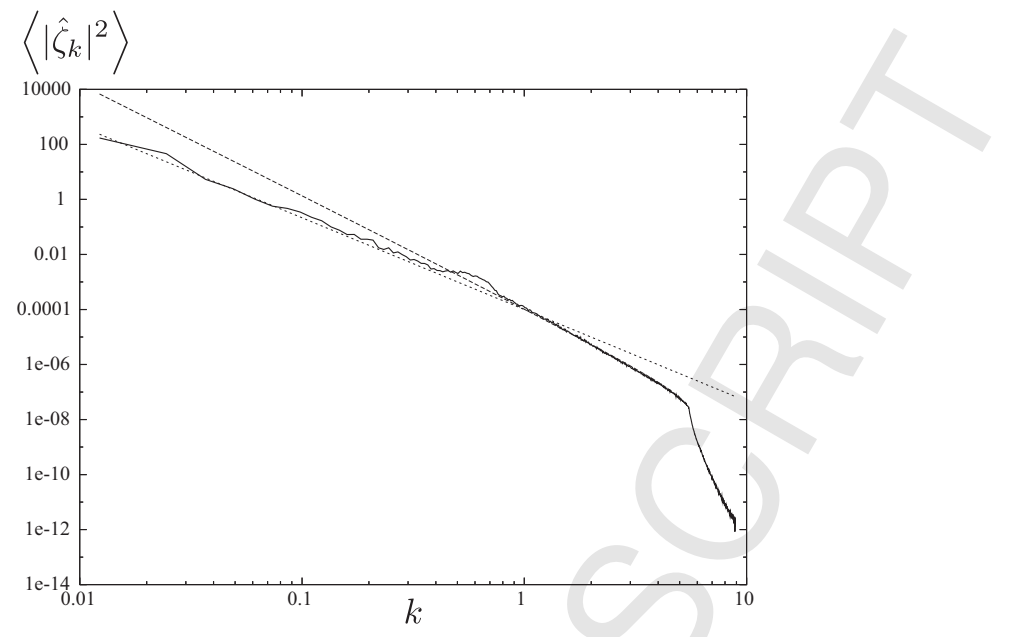

Figure 10: Displacement spectrum $\left\langle\left|\zeta_{\boldsymbol{k}}\right|^{2}\right\rangle$ as function of the wave number $k$ for an injection in an intermediate range $k_{i}=0.6$ with $\delta=0.2$. Dissipation is at small range $k_{d}=5.5$ and pumping at large scale $k_{m}=0.03$ so that two inertial ranges are observed. One at small scales between $k_{i}$ and $k_{d}$ so that a direct cascade can be observed, fitted with the expected $k^{-4}$ spectrum (dashed line), regardless the logarithmic correction, and one at large scale, between $k_{m}$ and $k_{i}$, corresponding to an inverse cascade regime, well fitted by a $k^{-10 / 3}$ law (dotted line).

\subsection{Strong turbulence in a plate, the limit $h \rightarrow 0$.}

The limit $h \rightarrow 0$ represents an interesting challenge for the WTT. Indeed, since the linear frequency is proportional to $h$, ( $\omega_{k} \propto h$ in the dispersion relation (3)), the linear time scale of the oscillation diverges which makes the WTT asymptotic limit not well defined. The dimensionless version of the equation involving length and time scales diverging when $h \rightarrow 0$, this limit can be seen as that of an infinite plate with infinite time scale. Moreover, in this limit, the nonlinear term becomes dominant over the linear ones, leading to the formal nonlinear plate dynamics:

$$
\frac{\partial^{2} \zeta}{\partial t^{2}}=-\frac{E}{2 \rho}\left\{\zeta, \Delta^{-2}\{\zeta, \zeta\}\right\}
$$

This dynamical model could be deduced fromthe theory of elasticity after imposing that the out-of-plane deformations are much larger than the in-plane deformations, and similarly for the speed of deformations, leading to the set of conditions $\left|u_{x}\right|,\left|u_{y}\right| \ll|\zeta|$ and $\left|\partial_{t} u_{x}\right|,\left|\partial_{t} u_{y}\right| \ll\left|\partial_{t} \zeta\right|$. Hence, the in-plane sound modes are neglected as in the original FvK equations.

Since no linear term are anymore present, turbulent dynamics in this limit can be called "strong" plate turbulence. In fact, we can consider that the oscillation frequency depends on the amplitude of the waves yielding $\nu_{k} \sim \sqrt{E / \rho} k^{2}\left|\zeta_{k}\right|$.

Following the dimensional analysis, two situations can be considered. Firstly, the general case, for which the spectrum is independent of $h$, leads to Eq. (10):

$$
E_{k}=\frac{E}{\rho k} \Phi_{2}\left(\frac{P}{(E / \rho)^{3 / 2} k}\right)
$$

In this formula the Young modulus $E$ is still present due to the relation of the Airy function with the Gaussian curvature (4). On the other hand, if, for some reasons, the Young modulus is not involved in the spectrum, indicating that the spectrum is material independent, the usual Kolmogorov spectrum for fluid turbulence is retrieved, Eq. (11):

$$
E_{k}=C P^{2 / 3} k^{-5 / 3}
$$

Therefore, further studies should investigate the vibration of a plate in the limit $h \rightarrow 0$ and for $h=0$ in order to observe which solution (and how) is selected by the dynamics. Moreover, since the nonlinearity is here cubic, instead of the quadratic one at play in fluid turbulence, this limit might help to characterize the role of the nonlinearity in the strong turbulence regime.

\subsection{Wave turbulence in cylindrical tubes and spherical shells}

The WTT that we have developed for elastic plates can be easily applied to other geometries such as elastic tubes or shells. If the technical aspect of the WTT can be adapted to these geometries, important differences in the results appear that need a 
specific discussion here. In fact, it introduces a new relevant parameter, namely $R$ the radius of the cylinder or the sphere ${ }^{6}$, that adds an extra dimensionless variable, $R / \ell$. Obviously, this additional parameter modifies the general dimensional analysis of the problem, but it affects in fact more profoundly the wave turbulence dynamics. Firstly, the inclusion of this new length modifies the dispersion relation, changing the power law dependence between the frequency and the wave-number. More precisely, the dispersion relation exhibits different behaviors in the small and large $k$ limits, questioning the possible expansion of the spectra into simple power laws. More importantly, the lowest order nonlinearities appears to be quadratic instead of cubic. For quadratic nonlinearity in general, WTT predicts that the relation between the energy spectrum and the energy flux $P$ should scale as $n_{k} \sim P^{1 / 2}$, instead of the $P^{1 / 3}$ found for cubic nonlinearity.

In the following, we present the results of the WTT applied to cylindrical tubes and spherical shells, emphasizing in particular the differences with the WTT for elastic plates..

\subsubsection{The Donnell-von Kármán equations.}

The extensions of the Föppl-von Kármán equations for a cylindrical tube with a radius $R$ which is large compared with the local amplitude of the shell deflection, the so-called Donnell-von Kármán equations, read [55, 56]

$$
\begin{aligned}
\rho \frac{\partial^{2} \zeta}{\partial t^{2}} & =-\frac{E h^{2}}{12\left(1-\sigma^{2}\right)} \Delta^{2} \zeta+\frac{1}{R} \partial_{x x} \chi+\{\zeta, \chi\} \\
\frac{1}{E} \Delta^{2} \chi & =-\frac{1}{R} \partial_{x x} \zeta-\frac{1}{2}\{\zeta, \zeta\} .
\end{aligned}
$$

where the $x$ and $y$ are the curvilinear coordinates running along the axial direction of the cylinder, and along the circumference respectively. The new term in the dynamics $\frac{1}{R} \partial_{x x} \chi$ introduces a quadratic nonlinearity through the relation (104). It should be emphasized that in the weak turbulence framework, this nonlinearity should dominate the cubic one of elastic plates, changing thus dramatically the wave interaction process. Moreover, the linear dispersion relation becomes then

$$
\omega_{\boldsymbol{k}}=\sqrt{\frac{E}{\rho}\left(\frac{h^{2}}{12\left(1-\sigma^{2}\right)} k^{4}+\frac{k_{x}^{4}}{R^{2} k^{4}}\right)},
$$

which is a combination of two power law behaviors, $\omega_{\boldsymbol{k}} \propto k^{2}$ at large $k$, (such that $k R \gg 1$ indicating that at these scales, the shell behaves as a plate) and $\omega_{\boldsymbol{k}} \propto C t e$ for small $k(k R \ll 1)$, with in this case a non-isotropic property of the relation. A cylindrical vibrating tube provides thus a non-isotropic oscillations which is reflected in the dynamical equations and in the dispersion relation. The isotropy is recovered in the case of a spherical cap, for which the equations of motion can be obtained in a simple way, yielding

$$
\frac{1}{R} \partial_{x x} \chi \longrightarrow \frac{1}{R} \Delta \chi \quad \text { in (103), and } \quad \frac{1}{R} \partial_{x x} \zeta \longrightarrow \frac{1}{R} \Delta \zeta \quad \text { in (104). }
$$

The linear dispersion relation is then:

$$
\omega_{\boldsymbol{k}}=\sqrt{\frac{E}{\rho}\left(\frac{h^{2}}{12\left(1-\sigma^{2}\right)} k^{4}+\frac{1}{R^{2}}\right)},
$$

that presents the same two asymptotic behaviors than for the tubes. In particular, notice that this dispersion relation exhibits a minimum frequency, $\omega_{0}=\sqrt{\frac{E}{\rho}} \frac{1}{R}$ and thus a forbiden frequency range $\left[-\omega_{0}, \omega_{0}\right]$ for the vibrations. Similarly to (12) and (13), the dimensionless versions of the Donnell-von Kármán equations for cylindrical tubes and elastic spherical shells read

$$
\begin{aligned}
\frac{\partial^{2} \zeta^{\prime}}{\partial t^{\prime 2}} & =-\frac{1}{4} \Delta^{2} \zeta^{\prime}+\frac{1}{R^{\prime}} \partial_{x x} \chi^{\prime}+\left\{\zeta^{\prime}, \chi^{\prime}\right\} \\
\Delta^{2} \chi^{\prime} & =-\frac{1}{R^{\prime}} \partial_{x x}^{\prime} \zeta-\frac{1}{2}\left\{\zeta^{\prime}, \zeta^{\prime}\right\} .
\end{aligned}
$$

where $R^{\prime}=R / \ell$ and again the primes will be dropped everywhere in the following.

\footnotetext{
${ }^{6}$ One may imagine that locally any shell may be seen as a combination of two distinct radius of curvature.
} 


\subsubsection{Three-waves kinetic equation for the case of shells.}

The nonlinear equations for the normal mode amplitudes reads:

$$
\frac{d A_{\boldsymbol{k}}^{s}}{d t}+i s \omega_{s \boldsymbol{k}} A_{\boldsymbol{k}}^{s}=\epsilon \sum_{s_{1} s_{2}} \int L_{\boldsymbol{k}_{1} \boldsymbol{k}_{2}}^{s s_{1} s_{2}} A_{\boldsymbol{k}_{1}}^{s_{1}} A_{\boldsymbol{k}_{2}}^{s_{2}} \delta\left(\boldsymbol{k}_{1}+\boldsymbol{k}_{2}-\boldsymbol{k}\right) d \boldsymbol{k}_{12}+\epsilon^{2} \sum_{s_{1} s_{2} s_{3}} \int L_{\boldsymbol{k k}_{1} \boldsymbol{k}_{2} \boldsymbol{k}_{3}}^{s s_{1} s_{2} s_{3}} A_{\boldsymbol{k}_{1}}^{s_{1}} A_{\boldsymbol{k}_{2}}^{s_{2}} A_{\boldsymbol{k}_{3}}^{s_{3}} \delta\left(\boldsymbol{k}_{1}+\boldsymbol{k}_{2}+\boldsymbol{k}_{3}-\boldsymbol{k}\right) d \boldsymbol{k}_{123},
$$

which generalizes the equation (29). Here $\omega_{\boldsymbol{k}}$ is the linear dispersion relation (105) for the cylindrical shell and of (107) for the spherical shell. The nonlinear interaction terms witness the quadratic nonlinearity and are given by:

$$
L_{\boldsymbol{k}_{1} \boldsymbol{k}_{2}}^{s s_{1} s_{2}}=-\frac{i s}{(2 \pi)} W_{-\boldsymbol{k} \boldsymbol{k}_{1} \boldsymbol{k}_{2}} \quad \text { with } \quad W_{\boldsymbol{k}_{1} \boldsymbol{k}_{2} \boldsymbol{k}_{3}}=\frac{3}{2 \sqrt{2}} X_{\boldsymbol{k}_{1}} X_{\boldsymbol{k}_{2}} X_{\boldsymbol{k}_{3}} V_{\boldsymbol{k}_{1} \boldsymbol{k}_{2} \boldsymbol{k}_{3}}
$$

together with $X_{\boldsymbol{k}}=1 / \sqrt{\omega_{\boldsymbol{k}}}$ and

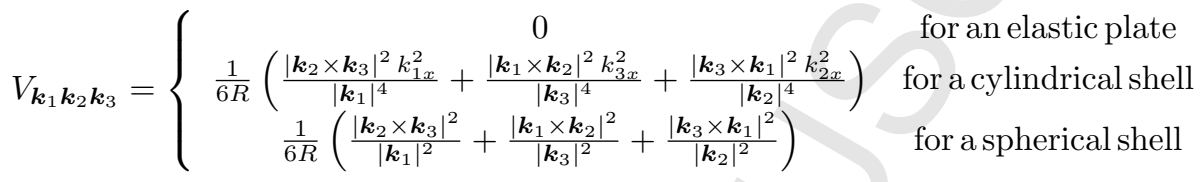

The cubic nonlinear terms are exactly the same as in the case of a plate. Notice that in the limit of $R \rightarrow \infty$ one recovers the equations for plates. Remarkably, both the dispersion relation $\omega_{\boldsymbol{k}}$ and the coefficients $V_{\boldsymbol{k}_{1} \boldsymbol{k}_{2} \boldsymbol{k}_{3}}$ are not isotropic in the case of a cylinder shell.

Because of the quadratic nonlinearity that should a priori dominate the cubic one, the first non trivial contribution in the WTT comes at the second order correction because of the three wave resonance induced by this quadratic interaction. This case has been largely discussed in the literature [4] and can be easily adapted for the shell dynamics leading to the kinetic equation:

$$
\begin{aligned}
\frac{d}{d t} n(\boldsymbol{p}) & =4 \pi \epsilon^{2} \int\left|W_{\boldsymbol{p} \boldsymbol{k}_{1} \boldsymbol{k}_{2}}\right|^{2} n(\boldsymbol{p}) n\left(\boldsymbol{k}_{1}\right) n\left(\boldsymbol{k}_{2}\right)\left(\frac{1}{n(\boldsymbol{p})}-\frac{1}{n\left(\boldsymbol{k}_{1}\right)}-\frac{1}{n\left(\boldsymbol{k}_{2}\right)}\right) \delta\left(\omega(p)-\omega\left(k_{1}\right)-\omega\left(k_{2}\right)\right) \delta\left(\boldsymbol{p}-\boldsymbol{k}_{1}-\boldsymbol{k}_{2}\right) d \boldsymbol{k}_{12} \\
& +4 \pi \epsilon^{2} \int\left|W_{\boldsymbol{p} \boldsymbol{k}_{1} \boldsymbol{k}_{2}}\right|^{2} n(\boldsymbol{p}) n\left(\boldsymbol{k}_{1}\right) n\left(\boldsymbol{k}_{2}\right)\left(\frac{1}{n(\boldsymbol{p})}+\frac{1}{n\left(\boldsymbol{k}_{1}\right)}-\frac{1}{n\left(\boldsymbol{k}_{2}\right)}\right) \delta\left(\omega(p)+\omega\left(k_{1}\right)-\omega\left(k_{2}\right)\right) \delta\left(\boldsymbol{p}+\boldsymbol{k}_{1}-\boldsymbol{k}_{2}\right) d \boldsymbol{k}_{12} \\
& +4 \pi \epsilon^{2} \int\left|W_{\boldsymbol{p} \boldsymbol{k}_{1} \boldsymbol{k}_{2}}\right|^{2} n(\boldsymbol{p}) n\left(\boldsymbol{k}_{1}\right) n\left(\boldsymbol{k}_{2}\right)\left(\frac{1}{n(\boldsymbol{p})}-\frac{1}{n\left(\boldsymbol{k}_{1}\right)}+\frac{1}{n\left(\boldsymbol{k}_{2}\right)}\right) \delta\left(\omega(p)-\omega\left(k_{1}\right)+\omega\left(k_{2}\right)\right) \delta\left(\boldsymbol{p}-\boldsymbol{k}_{1}+\boldsymbol{k}_{2}\right) d \boldsymbol{k}_{12}(11
\end{aligned}
$$

The four waves resonant due to the cubic interaction term is also present but appears at order $\epsilon^{4}$ in the expansion, so that for small enough $\epsilon$ the three wave interaction process is dominant.

\subsubsection{Basic properties.}

This three-waves kinetic equation (112) satisfies the same basic properties than the four-waves one obtained for elastic plates in Sec. 5.2, namely equation (112) conserves energy, $\int \omega_{\boldsymbol{k}} n_{\boldsymbol{k}} d \boldsymbol{k}$ and momentum $\int \boldsymbol{k} n_{\boldsymbol{k}} d \boldsymbol{k}$. Furthermore, the kinetic equation is mandated by a H-theorem leading an isolated dynamics towards an equilibrium.

\subsubsection{Kolmogorov-Zakharov spectrum for the case of shells.}

Remarkably, equation (112) does not exhibit a pure power law spectrum as a stationary solution. This does not imply that a wave turbulence spectrum does not exist, it just means that the stationary cascade is not a single power law. This is clearly a consequence of the fact that the dispersion relation is not a simple power law in $k$. However, equation (112) maybe simplified in the case of a spherical shell which corresponds to the case of isotropic spectrum. In this case the collisional term simplifies to:

$$
\frac{d}{d t} n_{p}=4 \pi \epsilon^{2} \int S_{\omega_{p} \omega_{1} \omega_{2}}\left[\left(n_{1} n_{2}-n_{p} n_{1}-n_{p} n_{2}\right) \delta\left(\omega_{p}-\omega_{1}-\omega_{2}\right)+2\left(n_{1} n_{2}+n_{p} n_{2}-n_{p} n_{1}\right) \delta\left(\omega_{p}+\omega_{1}-\omega_{2}\right)\right] d \omega_{1} d \omega_{2}
$$

with

$$
S_{\omega_{p} \omega_{1} \omega_{2}}=\frac{p}{\omega_{p}^{\prime}} \frac{k_{1}}{\omega_{1}^{\prime}} \frac{k_{2}}{\omega_{2}^{\prime}}\left(\int_{0}^{2 \pi}\left|W_{\boldsymbol{p} \boldsymbol{k}_{1} \boldsymbol{k}_{2}}\right|^{2} \delta\left(\boldsymbol{k}_{1}+\boldsymbol{k}_{2}+\boldsymbol{p}\right) d \varphi_{p} d \varphi_{k_{1}} d \varphi_{k_{2}}\right)
$$


Here $\omega_{p}^{\prime}$ stands for the group velocity of the wave $d \omega_{p} / d p$. Kolmogorov power law spectra can then be derived in the large $R$ limit $(R / \ell \gg 1)$. In this case the dispersion relation (107) corresponds to $\omega_{k} \approx \frac{1}{2} k^{2}+\frac{1}{k^{2} R^{2}}+\ldots$ Keeping the ballistic behavior only $\omega_{k} \sim \frac{1}{2} k^{2}$, one can deduce from (114) that $S_{\omega_{p} \omega_{1} \omega_{2}} \sim \frac{1}{\omega^{2} R^{2}}$. It is thus possible in this limit to seek a power law solution for the spectra: $n_{\omega_{p}}=K \omega_{p}{ }^{-x}$. In this case the collisional term becomes:

$$
\operatorname{Coll}_{2 \leftrightarrow 1}[n]=K^{2} \omega_{p}^{-2 x-1} I_{2 \leftrightarrow 1}(x) .
$$

Here $I_{2 \leftrightarrow 1}(x)$ is a function of $x$, that scales as $1 / R^{2}$. The dimensionless function $R^{2} I_{2 \leftrightarrow 1}(x)$ may be determined in principle numerically as in the case of 4 -wave interactions (Sec. 6.1), yielding

$$
I_{2 \leftrightarrow 1}(x)=4 \pi \int_{\mathcal{D}} S_{1 u_{1} u_{2}}\left(u_{1} u_{2}\right)^{-x}\left(1-u_{1}^{x}-u_{2}^{x}\right)\left(1-u_{1}^{2 x}-u_{2}^{2 x}\right) \delta\left(1-u_{1}-u_{2}\right) d u_{1} d u_{2} .
$$

The stationary solutions arises from the zeroes of the function $I_{2 \leftrightarrow 1}(x)$. This last one at least vanishes for $x=1$, the equilibrium Rayleigh-Jeans equipartition, and for $x=1 / 2$ which corresponds to finite energy flux stationary solution with a constant pre-factor $K^{2}=2 P\left(\left.\frac{\partial I_{2 \leftrightarrow 1}(x)}{\partial x}\right|_{x=1 / 2}\right)^{-1}$. The finite flux of energy stationary power law Kolmogorov spectrum

$$
n_{\omega} \sim P^{1 / 2} \frac{R}{\omega^{1 / 2}}
$$

is different than previous KZ spectrum (161) and different from the Rayleigh-Jeans equilibrium solution. However, the stationary solution with $x=1 / 2$ leads to a negative value for $\left.\frac{\partial I_{2 \leftrightarrow 1}(x)}{\partial x}\right|_{x=1 / 2}$, so that $K^{2}<0$. The reader may notice that the function $I_{2 \leftrightarrow 1}(x)$ has a positive concavity, therefore necessarily $\left.\frac{\partial I_{2 \leftrightarrow 1}(x)}{\partial x}\right|_{x=1}>0$ and $\left.\frac{\partial I_{2 \leftrightarrow 1}(x)}{\partial x}\right|_{x=1 / 2}<0$. Therefore, no stationary power law solution is expected to exist here aside from the Rayleigh-Jean distribution equilibrium, a prediction that would be worth to investigate in the future.

\section{Conclusions}

In this paper, we have presented a full derivation of the WTT for vibrating elastic plates. The analysis for a plate shows that the cubic nonlinearity and the dispersive property of the waves lead to a four-waves nonlinear interaction processes. In this case, the KZ turbulent solution is degenerated from the Rayleigh-Jeans distribution, so that the direct cascade of energy KZ spectrum ends up as a logarithmic correction of the Rayleigh-Jeans distribution. Furthermore, since the dynamics does not preserve the wave action no stationary inverse cascade solution can exist. Numerical simulations exhibit good agreement with the WTT. On the other hand, experimental turbulent spectra show important differences with the predictions of the WTT that can be mainly explained by the typical dissipation in plates that is present at all scales. Generalization of this WTT approach to shell geometries has been also derived involving a three-waves interaction process instead of the four-waves resonance for plates. Different perspectives of this work have been finally drawn in particular concerning the breakdown of wave turbulence theory and the limit $h \rightarrow 0$ of strong turbulence.

Furthermore, plate wave turbulence provides actually a convenient tool for investigating the various aspects and perspectives of wave turbulence. In fact, even if the four wave resonance exhibits degeneracy with the Rayleigh-Jean distribution, leading to a logarithmic correction, it represents a prototype of wave turbulence, since both experimental and numerical investigations are easy to perform, which is not the case for many wave turbulent systems. Recent theoretical and numerical developments have taken advantages of this situation to investigate the statistical properties of this wave turbulence [37, 34, 38], showing in particular an interesting connexion between the breakdown of wave turbulence at large scales with the appearance of intermittencies and $d$ cones, as explained above. Phenomenological models have also been developed, allowing to study more easily transient dynamics or realistic dissipation [29]. These models are based on the assumptions of the locality of the interactions in the Fourier space leading to a nonlinear diffusion equation in the spectral domain, equivalent the Leith equation for turbulence [60]. The nonlinear diffusion equation is obtained by imposing the Rayleigh-Jeans distribution and the KZ spectra as stationary solution although a complete derivation of such local equation in the spectral domain is still lacking. 


\section{Appendix}

\subsection{Relations between moments and cumulants}

In this Appendix we derive the one to one relation between the moments and cumulants in the real and in the Fourier spaces. If we denote the cumulants by the curly brackets $\{\cdots\}$, then the one to one moment-cumulant relations are:

$$
\begin{aligned}
\left\langle u^{s}(\boldsymbol{x})\right\rangle= & \left\{u^{s}(\boldsymbol{x})\right\} \\
\left\langle u^{s}(\boldsymbol{x}) u^{s_{1}}\left(\boldsymbol{x}+\boldsymbol{r}_{1}\right)\right\rangle= & \left\{u^{s}(\boldsymbol{x}) u^{s_{1}}\left(\boldsymbol{x}+\boldsymbol{r}_{1}\right)\right\}+\left\{u^{s}(\boldsymbol{x})\right\}\left\{u^{s_{1}}\left(\boldsymbol{r}_{1}\right)\right\} \\
\left\langle u^{s}(\boldsymbol{x}) u^{s_{1}}\left(\boldsymbol{x}+\boldsymbol{r}_{1}\right) u^{s_{2}}\left(\boldsymbol{x}+\boldsymbol{r}_{2}\right)\right\rangle= & \left\{u^{s}(\boldsymbol{x}) u^{s_{1}}\left(\boldsymbol{x}+\boldsymbol{r}_{1}\right) u^{s_{2}}\left(\boldsymbol{x}+\boldsymbol{r}_{2}\right)\right\}+ \\
& \left\{u^{s}(\boldsymbol{x})\right\}\left\{u^{s_{1}}\left(\boldsymbol{x}+\boldsymbol{r}_{1}\right) u^{s_{2}}\left(\boldsymbol{x}+\boldsymbol{r}_{2}\right)\right\}+\left\{u^{s_{1}}\left(\boldsymbol{x}+\boldsymbol{r}_{1}\right)\right\}\left\{u^{s}(\boldsymbol{x}) u^{s_{2}}\left(\boldsymbol{x}+\boldsymbol{r}_{2}\right)\right\}+ \\
& \left\{u^{s_{2}}\left(\boldsymbol{x}+\boldsymbol{r}_{2}\right)\right\}\left\{u^{s}(\boldsymbol{x}) u^{s_{1}}\left(\boldsymbol{x}+\boldsymbol{r}_{1}\right)\right\}+\left\{u^{s}(\boldsymbol{x})\right\}\left\{u^{s_{1}}\left(\boldsymbol{x}+\boldsymbol{r}_{1}\right)\right\}\left\{u^{s_{2}}\left(\boldsymbol{x}+\boldsymbol{r}_{2}\right)\right\} \\
\vdots & \\
\left\langle u^{s}(\boldsymbol{x}) u^{s_{1}}\left(\boldsymbol{x}+\boldsymbol{r}_{1}\right) \ldots u^{s_{n-1}}\left(\boldsymbol{x}+\boldsymbol{r}_{n-1}\right)\right\rangle= & \left\{u^{s}(\boldsymbol{x}) u^{s_{1}}\left(\boldsymbol{x}+\boldsymbol{r}_{1}\right) \ldots u^{s_{n-1}}\left(\boldsymbol{x}+\boldsymbol{r}_{n-1}\right)\right\}+\text { one of all possible partitions. }
\end{aligned}
$$

Here by "one of all possible partitions" we stand

$$
\left\{a_{1} a_{2} a_{3} \ldots a_{n}\right\}=\left\{a_{1}\right\}\left\{a_{2} a_{3} \ldots a_{n}\right\}+\left\{a_{2}\right\}\left\{a_{1} a_{3} \ldots a_{n}\right\}+\cdots+\left\{a_{1} a_{2}\right\}\left\{a_{3} \ldots a_{n}\right\}+\cdots+\left\{a_{1}\right\}\left\{a_{2}\right\} \ldots\left\{a_{n}\right\} .
$$

Because of the space homogeneity, that is the cumulants and the moments do not depend on the absolute spatial positions but only on the relative geometry. Hence, it is convenient to write them as follows:

$$
R^{(n) s s_{1} \cdots s_{n-1}}\left(\boldsymbol{r}_{1}, \ldots, \boldsymbol{r}_{n-1}\right)=\left\{u^{s}(\boldsymbol{x}) u^{s_{1}}\left(\boldsymbol{x}+\boldsymbol{r}_{1}\right) \ldots u^{s_{n-1}}\left(\boldsymbol{x}+\boldsymbol{r}_{n-1}\right)\right\} .
$$

Since $\left\{u^{s_{i}}\left(\boldsymbol{x}+\boldsymbol{r}_{i}\right) u^{s_{j}}\left(\boldsymbol{x}+\boldsymbol{r}_{j}\right)\right\}=\left\{u^{s_{i}}(\boldsymbol{x}) u^{s_{j}}\left(\boldsymbol{x}+\boldsymbol{r}_{j}-\boldsymbol{r}_{i}\right)\right\}$, the moment-cumulant relation reads

$$
\begin{aligned}
\left\langle u^{s}(\boldsymbol{x})\right\rangle & =R^{(1) s}, \\
\left\langle u^{s}(\boldsymbol{x}) u^{s_{1}}\left(\boldsymbol{x}+\boldsymbol{r}_{1}\right)\right\rangle= & R^{(2) s s_{1}}\left(\boldsymbol{r}_{1}\right)+R^{(1) s} R^{(1) s_{1}}, \\
\left\langle u^{s}(\boldsymbol{x}) u^{s_{1}}\left(\boldsymbol{x}+\boldsymbol{r}_{1}\right) u^{s_{2}}\left(\boldsymbol{x}+\boldsymbol{r}_{2}\right)\right\rangle= & R^{(3) s s_{1} s_{2}}\left(\boldsymbol{r}_{1}, \boldsymbol{r}_{2}\right)+R^{(1) s} R^{(2) s_{1} s_{2}}\left(\boldsymbol{r}_{2}-\boldsymbol{r}_{1}\right)+ \\
& R^{(1) s_{1}} R^{(2) s s_{2}}\left(\boldsymbol{r}_{2}\right)+R^{(1) s_{2}} R^{(2) s s_{1}}\left(\boldsymbol{r}_{1}\right)+R^{(1) s} R^{(1) s_{1}} R^{(1) s_{2}}, \\
\left\langle u^{s}(\boldsymbol{x}) u^{s_{1}}\left(\boldsymbol{x}+\boldsymbol{r}_{1}\right) u^{s_{2}}\left(\boldsymbol{x}+\boldsymbol{r}_{2}\right) u^{s_{3}}\left(\boldsymbol{x}+\boldsymbol{r}_{3}\right)\right\rangle= & R^{(4) s s_{1} s_{2} s_{3}}\left(\boldsymbol{r}_{1}, \boldsymbol{r}_{2}, \boldsymbol{r}_{3}\right)+R^{(1) s} R^{(3) s_{1} s_{2} s_{3}}\left(\boldsymbol{r}_{2}-\boldsymbol{r}_{1}, \boldsymbol{r}_{3}-\boldsymbol{r}_{1}\right)+ \\
& \mathcal{P}_{123} R^{(1) s_{1}} R^{(3) s s_{2} s_{3}}\left(\boldsymbol{r}_{2}, \boldsymbol{r}_{3}\right)+\mathcal{P}_{123} R^{(2) s s_{1}}\left(\boldsymbol{r}_{1}\right) R^{(2) s_{2} s_{3}}\left(\boldsymbol{r}_{3}-\boldsymbol{r}_{2}\right) \\
& \mathcal{P}_{123} R^{(1) s_{1}} R^{(1) s_{2}} R^{(2) s s_{3}}\left(\boldsymbol{r}_{3}\right)+\mathcal{P}_{123} R^{(1) s} R^{(1) s_{1}} R^{(2) s_{2} s_{3}}\left(\boldsymbol{r}_{3}-\boldsymbol{r}_{2}\right)+ \\
& R^{(1) s} R^{(1) s_{1}} R^{(1) s_{2}} R^{(1) s_{3}}, \\
\left\langle u^{s}(\boldsymbol{x}) u^{s_{1}}\left(\boldsymbol{x}+\boldsymbol{r}_{1}\right) \ldots u^{s_{n-1}}\left(\boldsymbol{x}+\boldsymbol{r}_{n-1}\right)\right\rangle= & R^{(n) s s_{1} \cdots s_{n-1}}\left(\boldsymbol{r}_{1}, \ldots, \boldsymbol{r}_{n-1}\right)+\text { one of all possible partitions. }
\end{aligned}
$$

Where $R^{(1) s}$ does not depend on the space variable, but only on the temporal ones. The symbol $\mathcal{P}_{123}$ stands for a cyclical permutation over the indices $1,2,3$ (i.e. $\left.\mathcal{P}_{123} B_{123}=B_{123}+B_{312}+B_{231}\right)$.

We end this Appendix by adding also the expressions for the higher order moments in the Fourier space. Replacing the relations obtained in (119), and using (35) one obtains that the $n$-th order moment in the Fourier space is directly related to the cumulants up to $n$-th order by: 


$$
\begin{aligned}
& \left\langle A_{\boldsymbol{p}_{1}}^{l_{1}}\right\rangle=(2 \pi) Q^{(1) l_{1}} \delta\left(\boldsymbol{p}_{1}\right) \\
& \left\langle A_{\boldsymbol{p}_{1}}^{l_{1}} A_{\boldsymbol{p}_{2}}^{l_{2}}\right\rangle=(2 \pi) Q^{(2) l_{1} l_{2}}\left(\boldsymbol{p}_{2}\right) \delta\left(\boldsymbol{p}_{1}+\boldsymbol{p}_{2}\right) \\
& \left\langle A_{\boldsymbol{k}_{1}}^{s_{1}} A_{\boldsymbol{k}_{2}}^{s_{2}} A_{\boldsymbol{k}_{3}}^{s_{3}}\right\rangle=(2 \pi) Q^{(3) s_{1} s_{2} s_{3}}\left(\boldsymbol{k}_{2}, \boldsymbol{k}_{3}\right) \delta\left(\boldsymbol{k}_{1}+\boldsymbol{k}_{2}+\boldsymbol{k}_{3}\right) \\
& \left\langle A_{\boldsymbol{p}_{1}}^{l_{1}} A_{\boldsymbol{k}_{1}}^{s_{1}} A_{\boldsymbol{k}_{2}}^{s_{2}} A_{\boldsymbol{k}_{3}}^{s_{3}}\right\rangle=(2 \pi) Q^{(4) l_{1} s_{1} s_{2} s_{3}}\left(\boldsymbol{k}_{1}, \boldsymbol{k}_{2}, \boldsymbol{k}_{3}\right) \delta\left(\boldsymbol{p}_{1}+\boldsymbol{k}_{1}+\boldsymbol{k}_{2}+\boldsymbol{k}_{3}\right)+ \\
& +(2 \pi)^{2} \mathcal{P}_{123} Q^{(2) l_{1} s_{1}}\left(\boldsymbol{k}_{1}\right) Q^{(2) s_{2} s_{3}}\left(\boldsymbol{k}_{3}\right) \delta\left(\boldsymbol{p}_{1}+\boldsymbol{k}_{1}\right) \delta\left(\boldsymbol{k}_{2}+\boldsymbol{k}_{3}\right) \\
& \left\langle A_{\boldsymbol{p}_{1}}^{l_{1}} A_{\boldsymbol{p}_{2}}^{l_{2}} A_{\boldsymbol{k}_{1}}^{s_{1}} A_{\boldsymbol{k}_{2}}^{s_{2}} A_{\boldsymbol{k}_{3}}^{s_{3}}\right\rangle=(2 \pi) Q^{(5) l_{1} l_{2} s_{1} s_{2} s_{3}}\left(\boldsymbol{p}_{2}, \boldsymbol{k}_{1}, \boldsymbol{k}_{2}, \boldsymbol{k}_{3}\right) \delta\left(\boldsymbol{p}_{1}+\boldsymbol{p}_{2}+\boldsymbol{k}_{1}+\boldsymbol{k}_{2}+\boldsymbol{k}_{3}\right)+ \\
& +\quad(2 \pi)^{2} \mathcal{P}_{1^{\prime} 2^{\prime}} \mathcal{P}_{123} Q^{(2) l_{1} s_{1}}\left(\boldsymbol{k}_{1}\right) Q^{(3) l_{2} s_{2} s_{3}}\left(\boldsymbol{k}_{2}, \boldsymbol{k}_{3}\right) \delta\left(\boldsymbol{p}_{1}+\boldsymbol{k}_{1}\right) \delta\left(\boldsymbol{p}_{2}+\boldsymbol{k}_{2}+\boldsymbol{k}_{3}\right)+ \\
& +(2 \pi)^{2} Q^{(2) l_{1} l_{2}}\left(\boldsymbol{p}_{2}\right) Q^{(3) s_{1} s_{2} s_{3}}\left(\boldsymbol{k}_{2}, \boldsymbol{k}_{3}\right) \delta\left(\boldsymbol{p}_{1}+\boldsymbol{p}_{2}\right) \delta\left(\boldsymbol{k}_{1}+\boldsymbol{k}_{2}+\boldsymbol{k}_{3}\right)+ \\
& +(2 \pi)^{2} \mathcal{P}_{123} Q^{(2) s_{1} s_{2}}\left(\boldsymbol{k}_{2}\right) Q^{(3) l_{1} l_{2} s_{3}}\left(\boldsymbol{p}_{2}, \boldsymbol{k}_{3}\right) \delta\left(\boldsymbol{k}_{1}+\boldsymbol{k}_{2}\right) \delta\left(\boldsymbol{p}_{1}+\boldsymbol{p}_{2}+\boldsymbol{k}_{3}\right) \\
& \left\langle A_{\boldsymbol{p}_{1}}^{l_{1}} A_{\boldsymbol{p}_{2}}^{l_{2}} A_{\boldsymbol{p}_{3}}^{l_{3}} A_{\boldsymbol{k}_{1}}^{s_{1}} A_{\boldsymbol{k}_{2}}^{s_{2}} A_{\boldsymbol{k}_{3}}^{s_{3}}\right\rangle=(2 \pi) Q^{(6) l_{1} l_{2} l_{3} s_{1} s_{2} s_{3}}\left(\boldsymbol{p}_{2}, \boldsymbol{p}_{3}, \boldsymbol{k}_{1}, \boldsymbol{k}_{2}, \boldsymbol{k}_{3}\right) \delta\left(\boldsymbol{p}_{1}+\boldsymbol{p}_{2}+\boldsymbol{p}_{3}+\boldsymbol{k}_{1}+\boldsymbol{k}_{2}+\boldsymbol{k}_{3}\right)+ \\
& +(2 \pi)^{2} \mathcal{P}_{1^{\prime} 2^{\prime} 3^{\prime}} Q^{(2) l_{1} l_{2}}\left(\boldsymbol{p}_{2}\right) Q^{(4) l_{3} s_{1} s_{2} s_{3}}\left(\boldsymbol{k}_{1}, \boldsymbol{k}_{2}, \boldsymbol{k}_{3}\right) \delta\left(\boldsymbol{p}_{1}+\boldsymbol{p}_{2}\right) \delta\left(\boldsymbol{p}_{3}+\boldsymbol{k}_{1}+\boldsymbol{k}_{2}+\boldsymbol{k}_{3}\right)+ \\
& +(2 \pi)^{2} \mathcal{P}_{1^{\prime} 2^{\prime} 3^{\prime}} \mathcal{P}_{123} Q^{(2) l_{1} s_{1}}\left(\boldsymbol{k}_{1}\right) Q^{(4) l_{2} l_{3} s_{2} s_{3}}\left(\boldsymbol{p}_{3}, \boldsymbol{k}_{2}, \boldsymbol{k}_{3}\right) \delta\left(\boldsymbol{p}_{1}+\boldsymbol{k}_{1}\right) \delta\left(\boldsymbol{p}_{2}+\boldsymbol{p}_{3}+\boldsymbol{k}_{2}+\boldsymbol{k}_{3}\right)+ \\
& +(2 \pi)^{2} \mathcal{P}_{123} Q^{(2) s_{1} s_{2}}\left(\boldsymbol{k}_{2}\right) Q^{(4) l_{1} l_{2} l_{3} s_{3}}\left(\boldsymbol{p}_{2}, \boldsymbol{p}_{3}, \boldsymbol{k}_{3}\right) \delta\left(\boldsymbol{k}_{1}+\boldsymbol{k}_{2}\right) \delta\left(\boldsymbol{p}_{1}+\boldsymbol{p}_{2}+\boldsymbol{p}_{3}+\boldsymbol{k}_{3}\right)+ \\
& +\quad(2 \pi)^{2} \mathcal{P}_{1^{\prime} 2^{\prime} 3^{\prime}} \mathcal{P}_{123} Q^{(3) l_{1} l_{2} s_{1}}\left(\boldsymbol{p}_{2}, \boldsymbol{k}_{1}\right) Q^{(3) l_{3} s_{2} s_{3}}\left(\boldsymbol{k}_{2}, \boldsymbol{k}_{3}\right) \delta\left(\boldsymbol{p}_{1}+\boldsymbol{p}_{2}+\boldsymbol{k}_{1}\right) \delta\left(\boldsymbol{p}_{3}+\boldsymbol{k}_{2}+\boldsymbol{k}_{3}\right)+ \\
& +\quad(2 \pi)^{2} Q^{(3) l_{1} l_{2} l_{3}}\left(\boldsymbol{p}_{2}, \boldsymbol{p}_{3}\right) Q^{(3) s_{1} s_{2} s_{3}}\left(\boldsymbol{k}_{2}, \boldsymbol{k}_{3}\right) \delta\left(\boldsymbol{p}_{1}+\boldsymbol{p}_{2}+\boldsymbol{p}_{3}\right) \delta\left(\boldsymbol{k}_{1}+\boldsymbol{k}_{2}+\boldsymbol{k}_{3}\right)+ \\
& +\quad(2 \pi)^{3} \mathcal{P}_{1^{\prime} 2^{\prime} 3^{\prime}} \mathcal{P}_{123} Q^{(2) l_{1} l_{2}}\left(\boldsymbol{p}_{2}\right) Q^{(2) l_{3} s_{1}}\left(\boldsymbol{k}_{1}\right) Q^{(2) s_{2} s_{3}}\left(\boldsymbol{k}_{3}\right) \delta\left(\boldsymbol{p}_{1}+\boldsymbol{p}_{2}\right) \delta\left(\boldsymbol{p}_{3}+\boldsymbol{k}_{1}\right) \delta\left(\boldsymbol{k}_{2}+\boldsymbol{k}_{3}\right)+ \\
& +(2 \pi)^{3} \mathcal{P} \mathcal{T}_{123} Q^{(2) l_{1} s_{1}}\left(\boldsymbol{k}_{1}\right) Q^{(2) l_{2} s_{2}}\left(\boldsymbol{k}_{2}\right) Q^{(2) l_{3} s_{3}}\left(\boldsymbol{k}_{3}\right) \delta\left(\boldsymbol{p}_{1}+\boldsymbol{k}_{1}\right) \delta\left(\boldsymbol{p}_{2}+\boldsymbol{k}_{2}\right) \delta\left(\boldsymbol{p}_{3}+\boldsymbol{k}_{3}\right) \text {. }
\end{aligned}
$$

Where $\mathcal{P} \mathcal{T}_{123}$ stands by the all posible permutations over the indices 1,2 and 3.

\subsection{Computation of the cumulant hierarchy}

From the linear moments hierarchy (39) we can construct an equivalent non linear cumulant hierarchy using the relations (38) and (120) derived previously. After some algebra one gets for $n=2$

$$
\begin{aligned}
\frac{d}{d t} Q^{(2) l_{1} l_{2}}\left(\boldsymbol{p}_{2}\right) & =-i\left(l_{1} \omega\left(\boldsymbol{p}_{1}\right)+l_{2} \omega\left(\boldsymbol{p}_{2}\right)\right) Q^{(2) l_{1} l_{2}}\left(\boldsymbol{p}_{2}\right)+ \\
& +\epsilon^{2} \mathcal{P}_{1^{\prime} 2^{\prime}} \sum_{s_{1} s_{2} s_{3}} \int L_{\boldsymbol{p}_{2} \boldsymbol{k}_{1} \boldsymbol{k}_{2} \boldsymbol{k}_{3}}^{l_{2} s_{1} s_{2} s_{3}} Q^{(4) l_{1} s_{1} s_{2} s_{3}}\left(\boldsymbol{k}_{1}, \boldsymbol{k}_{2}, \boldsymbol{k}_{3}\right) \delta\left(\boldsymbol{p}_{1}+\boldsymbol{k}_{1}+\boldsymbol{k}_{2}+\boldsymbol{k}_{3}\right) d \boldsymbol{k}_{123}+ \\
& +(2 \pi) \epsilon^{2} \mathcal{P}_{1^{\prime} 2^{\prime}} \sum_{s_{1} s_{2} s_{3}} \int L_{\boldsymbol{p}_{2} \boldsymbol{k}_{1} \boldsymbol{k}_{2} \boldsymbol{k}_{3}}^{l_{2} s_{1} s_{3} s_{3}} \mathcal{P}_{123} Q^{(2) l_{1} s_{1}}\left(\boldsymbol{k}_{1}\right) Q^{(2) s_{2} s_{3}}\left(\boldsymbol{k}_{3}\right) \delta\left(\boldsymbol{p}_{1}+\boldsymbol{k}_{1}\right) \delta\left(\boldsymbol{k}_{2}+\boldsymbol{k}_{3}\right) d \boldsymbol{k}_{123}
\end{aligned}
$$

Though, the equation for the third order cumulant is not required for the case of planar plates, we write explicitly this equation because it becomes pertinent in the case of elastic shells [4]:

$$
\begin{aligned}
\frac{d}{d t} Q^{(3) l_{1} l_{2} l_{3}}\left(\boldsymbol{p}_{2}, \boldsymbol{p}_{3}\right) & =-i\left(l_{1} \omega\left(\boldsymbol{p}_{1}\right)+l_{2} \omega\left(\boldsymbol{p}_{2}\right)+l_{3} \omega\left(\boldsymbol{p}_{3}\right)\right) Q^{(3) l_{1} l_{2} l_{3}}\left(\boldsymbol{p}_{2}, \boldsymbol{p}_{3}\right) \\
& +\epsilon^{2} \mathcal{P}_{1^{\prime} 2^{\prime} 3^{\prime}} \sum_{s_{1} s_{2} s_{3}} \int L_{\boldsymbol{p}_{3} \boldsymbol{k}_{1} \boldsymbol{k}_{2} \boldsymbol{k}_{3}}^{l_{3} s_{1} s_{2} s_{3}} Q^{(5) l_{1} l_{2} s_{1} s_{2} s_{3}}\left(\boldsymbol{p}_{2}, \boldsymbol{k}_{1}, \boldsymbol{k}_{2}, \boldsymbol{k}_{3}\right) \delta\left(\boldsymbol{p}_{1}+\boldsymbol{p}_{2}+\boldsymbol{k}_{1}+\boldsymbol{k}_{2}+\boldsymbol{k}_{3}\right) d \boldsymbol{k}_{123} \\
& +\epsilon^{2} \mathcal{P}_{1^{\prime} 2^{\prime} 3^{\prime}} \sum_{s_{1} s_{2} s_{3}} \int L_{\boldsymbol{p}_{3} \boldsymbol{k}_{1} \boldsymbol{k}_{2} \boldsymbol{k}_{3}}^{l_{3} s_{1} s_{2} s_{3} \mathcal{P}^{\prime}{ }^{\prime}} \mathcal{P}_{123}(2 \pi) Q^{(2) l_{1} s_{1}}\left(\boldsymbol{k}_{1}\right) Q^{(3) l_{2} s_{2} s_{3}}\left(\boldsymbol{k}_{2}, \boldsymbol{k}_{3}\right) \delta\left(\boldsymbol{p}_{1}+\boldsymbol{k}_{1}\right) \delta\left(\boldsymbol{p}_{2}+\boldsymbol{k}_{2}+\boldsymbol{k}_{3}\right) d \boldsymbol{k}_{123} \\
& +\epsilon^{2} \mathcal{P}_{1^{\prime} 2^{\prime} 3^{\prime}} \sum_{s_{1} s_{2} s_{3}} \int L_{\boldsymbol{p}_{3} \boldsymbol{k}_{1} \boldsymbol{k}_{2} \boldsymbol{k}_{3}}^{l_{3} s_{1} s_{2} s_{3}} \mathcal{P}_{123}(2 \pi) Q^{(2) s_{1} s_{2}}\left(\boldsymbol{k}_{2}\right) Q^{(3) l_{1} l_{2} s_{3}}\left(\boldsymbol{p}_{2}, \boldsymbol{k}_{3}\right) \delta\left(\boldsymbol{k}_{1}+\boldsymbol{k}_{2}\right) \delta\left(\boldsymbol{p}_{1}+\boldsymbol{p}_{2}+\boldsymbol{k}_{3}\right) d \boldsymbol{k}_{123} \cdot(121)
\end{aligned}
$$


For $n=4$ one obtains:

$$
\begin{aligned}
& \frac{d}{d t} Q^{(4) l_{1} l_{2} l_{3} l_{4}}\left(\boldsymbol{p}_{2}, \boldsymbol{p}_{3}, \boldsymbol{p}_{4}\right)=-i\left(l_{1} \omega\left(\boldsymbol{p}_{1}\right)+l_{2} \omega\left(\boldsymbol{p}_{2}\right)+l_{3} \omega\left(\boldsymbol{p}_{3}\right)+l_{4} \omega\left(\boldsymbol{p}_{4}\right)\right) Q^{(4) l_{1} l_{2} l_{3} l_{4}}\left(\boldsymbol{p}_{2}, \boldsymbol{p}_{3}, \boldsymbol{p}_{4}\right)+ \\
& +\epsilon^{2} \mathcal{P}_{1^{\prime} 2^{\prime} 3^{\prime} 4^{\prime}} \sum_{s_{1} s_{2} s_{3}} \int L_{\boldsymbol{p}_{4} \boldsymbol{k}_{1} \boldsymbol{k}_{2} \boldsymbol{k}_{3}}^{l_{1} s_{1} s_{2} s_{3}} Q^{(6) l_{1} l_{2} l_{3} s_{1} s_{2} s_{3}}\left(\boldsymbol{p}_{2}, \boldsymbol{p}_{3}, \boldsymbol{k}_{1}, \boldsymbol{k}_{2}, \boldsymbol{k}_{3}\right) \delta\left(\boldsymbol{p}_{1}+\boldsymbol{p}_{2}+\boldsymbol{p}_{3}+\boldsymbol{k}_{1}+\boldsymbol{k}_{2}+\boldsymbol{k}_{3}\right) d \boldsymbol{k}_{123}+ \\
& +\quad(2 \pi) \epsilon^{2} \mathcal{P}_{1^{\prime} 2^{\prime} 3^{\prime} 4^{\prime}} \sum_{s_{1} s_{2} s_{3}} \int L_{\boldsymbol{p}_{4} \boldsymbol{k}_{1} \boldsymbol{k}_{2} \boldsymbol{k}_{3}}^{l_{4} s_{1} s_{2} s_{3}} \mathcal{P}_{1^{\prime} 2^{\prime} 3^{\prime}} \mathcal{P}_{123} Q^{(2) l_{1} s_{1}}\left(\boldsymbol{k}_{1}\right) Q^{(4) l_{2} l_{3} s_{2} s_{3}}\left(\boldsymbol{p}_{3}, \boldsymbol{k}_{2}, \boldsymbol{k}_{3}\right) \delta\left(\boldsymbol{p}_{1}+\boldsymbol{k}_{1}\right) \delta\left(\boldsymbol{p}_{2}+\boldsymbol{p}_{3}+\boldsymbol{k}_{2}+\boldsymbol{k}_{3}\right) d \boldsymbol{k}_{123}+ \\
& +\quad(2 \pi) \epsilon^{2} \mathcal{P}_{1^{\prime} 2^{\prime} 3^{\prime} 4^{\prime}} \sum_{s_{1} s_{2} s_{3}} \int L_{\boldsymbol{p}_{4} \boldsymbol{k}_{1} \boldsymbol{k}_{2} \boldsymbol{k}_{3}}^{l_{4} s_{1} s_{2} s_{3}} \mathcal{P}_{123} Q^{(2) s_{1} s_{2}}\left(\boldsymbol{k}_{2}\right) Q^{(4) l_{1} l_{2} l_{3} s_{3}}\left(\boldsymbol{p}_{2}, \boldsymbol{p}_{3}, \boldsymbol{k}_{3}\right) \delta\left(\boldsymbol{k}_{1}+\boldsymbol{k}_{2}\right) \delta\left(\boldsymbol{p}_{1}+\boldsymbol{p}_{2}+\boldsymbol{p}_{3}+\boldsymbol{k}_{3}\right) d \boldsymbol{k}_{123}+ \\
& +\quad(2 \pi) \epsilon^{2} \mathcal{P}_{1^{\prime} 2^{\prime} 3^{\prime} 4^{\prime}} \sum_{s_{1} s_{2} s_{3}} \int L_{\boldsymbol{p}_{4} \boldsymbol{k}_{1} \boldsymbol{k}_{2} \boldsymbol{k}_{3}}^{l_{4} s_{1} s_{2} s_{3}} \mathcal{P}_{1^{\prime} 2^{\prime} 3^{\prime}} \mathcal{P}_{123} Q^{(3) l_{1} l_{2} s_{1}}\left(\boldsymbol{p}_{2}, \boldsymbol{k}_{1}\right) Q^{(3) l_{3} s_{2} s_{3}}\left(\boldsymbol{k}_{2}, \boldsymbol{k}_{3}\right) \delta\left(\boldsymbol{p}_{1}+\boldsymbol{p}_{2}+\boldsymbol{k}_{1}\right) \delta\left(\boldsymbol{p}_{3}+\boldsymbol{k}_{2}+\boldsymbol{k}_{3}\right) d \boldsymbol{k}_{123}+ \\
& +(2 \pi)^{2} \epsilon^{2} \mathcal{P}_{1^{\prime} 2^{\prime} 3^{\prime} 4^{\prime}} \sum_{s_{1} s_{2} s_{3}} \int L_{\boldsymbol{p}_{4} \boldsymbol{k}_{1} \boldsymbol{k}_{2} \boldsymbol{k}_{3}}^{l_{1} s_{1} s_{2} s_{3}} \mathcal{P} \mathcal{T}_{123} Q^{(2) l_{1} s_{1}}\left(\boldsymbol{k}_{1}\right) Q^{(2) l_{2} s_{2}}\left(\boldsymbol{k}_{2}\right) Q^{(2) l_{3} s_{3}}\left(\boldsymbol{k}_{3}\right) \delta\left(\boldsymbol{p}_{1}+\boldsymbol{k}_{1}\right) \delta\left(\boldsymbol{p}_{2}+\boldsymbol{k}_{2}\right) \delta\left(\boldsymbol{p}_{3}+\boldsymbol{k}_{3}\right) d \boldsymbol{k}_{123},(12
\end{aligned}
$$

keeping in mind that for each equation $\sum_{i=1}^{n} \boldsymbol{p}_{i}=0$.

10.3. The order $\epsilon^{2}$ correction for the second order cumulant.

The equation for the second order cumulant (40) up to second order reads, after the change of variables (43) and keeping in mind the multi-scale assumption (42):

$$
\begin{aligned}
\frac{d}{d t} q_{2}^{(2) l_{1} l_{2}}\left(\boldsymbol{p}_{2}\right) & =-F_{2}^{(2) l_{1} l_{2}}\left(\boldsymbol{p}_{2}\right)+ \\
& +\mathcal{P}_{1^{\prime} 2^{\prime}} \sum_{s_{1} s_{2} s_{3}} \int L_{\boldsymbol{p}_{2} \boldsymbol{k}_{1} \boldsymbol{k}_{2} \boldsymbol{k}_{3}}^{l_{2} s_{1} s_{2} s_{3}} q_{0}^{(4) l_{1} s_{1} s_{2} s_{3}}\left(\boldsymbol{k}_{1}, \boldsymbol{k}_{2}, \boldsymbol{k}_{3}\right) e^{i\left(l_{2} \omega\left(\boldsymbol{p}_{2}\right)-s_{1} \omega\left(\boldsymbol{k}_{1}\right)-s_{2} \omega\left(\boldsymbol{k}_{2}\right)-s_{3} \omega\left(\boldsymbol{k}_{3}\right)\right) t} \delta\left(\boldsymbol{p}_{1}+\boldsymbol{k}_{1}+\boldsymbol{k}_{2}+\boldsymbol{k}_{3}\right) d \boldsymbol{k}_{123}+ \\
& +(2 \pi) \mathcal{P}_{1^{\prime} 2^{\prime}} \sum_{s_{1} s_{2} s_{3}} \int L_{\boldsymbol{p}_{2} \boldsymbol{k}_{1} \boldsymbol{k}_{2} \boldsymbol{k}_{3}}^{l_{2} s_{1} s_{3} s_{3}} \mathcal{P}_{123} q_{0}^{(2) l_{1} s_{1}}\left(\boldsymbol{k}_{1}\right) q_{0}^{(2) s_{2} s_{3}}\left(\boldsymbol{k}_{3}\right) e^{i\left(l_{2} \omega\left(\boldsymbol{p}_{2}\right)-s_{1} \omega\left(\boldsymbol{k}_{1}\right)-s_{2} \omega\left(\boldsymbol{k}_{2}\right)-s_{3} \omega\left(\boldsymbol{k}_{3}\right)\right) t} \delta\left(\boldsymbol{p}_{1}+\boldsymbol{k}_{1}\right) \delta\left(\boldsymbol{k}_{2}+\boldsymbol{k}_{3}\right) d \boldsymbol{k}_{123}
\end{aligned}
$$

here $\boldsymbol{p}_{1}+\boldsymbol{p}_{2}=0$.

Expanding the eight terms of the sum $\sum_{s_{1} s_{2} s_{3}}$, and using the symmetries of the interaction terms, we can deduce, after some algebra, up to order $\mathcal{O}\left(\epsilon^{2}\right)$ :

$$
\begin{aligned}
& F_{2}^{(2) l_{1} l_{2}}\left(\boldsymbol{p}_{2}\right)=-\frac{d}{d t} q_{2}^{(2) l_{1} l_{2}}\left(\boldsymbol{p}_{2}\right) \\
& +\mathcal{P}_{1^{\prime} 2^{\prime}} \sum_{s_{1} s_{2} s_{3}} \int L_{\boldsymbol{p}_{2} \boldsymbol{k}_{1} \boldsymbol{k}_{2} \boldsymbol{k}_{3}}^{l_{2} s_{1} s_{2} s_{3}} q_{0}^{(4) l_{1} s_{1} s_{2} s_{3}}\left(\boldsymbol{k}_{1}, \boldsymbol{k}_{2}, \boldsymbol{k}_{3}\right) e^{-i t \Omega_{k_{1} k_{2} k_{3} p_{2}}^{s_{1} s_{2} s_{3}-l_{2}} \delta\left(\boldsymbol{p}_{1}+\boldsymbol{k}_{1}+\boldsymbol{k}_{2}+\boldsymbol{k}_{3}\right) d \boldsymbol{k}_{123}} \\
& +\mathcal{P}_{1^{\prime} 2^{\prime}} \mathcal{P}_{123} \sum_{s_{2} s_{3}} \int L_{\boldsymbol{p}_{2} \boldsymbol{p}_{2}-\boldsymbol{k}_{3} \boldsymbol{k}_{3}}^{l_{2}-l_{2} s_{2} s_{3}}(2 \pi) q_{0}^{(2) s_{2} s_{3}}\left(\boldsymbol{k}_{3}\right) q_{0}^{(2) l_{1}-l_{2}}\left(\boldsymbol{p}_{2}\right) e^{-i t \Omega_{p_{2}-k_{3} k_{3} p_{2}}^{-l_{2} s_{2} s_{3}-l_{2}}} d \boldsymbol{k}_{3} \\
& +\mathcal{P}_{1^{\prime} 2^{\prime}} \mathcal{P}_{123} \sum_{s_{3}} \int L_{\boldsymbol{p}_{2} \boldsymbol{p}_{2}-\boldsymbol{k}_{3} \boldsymbol{k}_{3}}^{l_{2} l_{2} s_{3} s_{3}}(2 \pi) q_{0}^{(2) s_{3} s_{3}}\left(\boldsymbol{k}_{3}\right) q_{0}^{(2) l_{1} l_{2}}\left(\boldsymbol{p}_{2}\right) e^{-i t \Omega_{p_{2}-k_{3} k_{3} p_{2}}^{l_{2} s_{3} s_{3}-l_{2}}} d \boldsymbol{k}_{3} \\
& +\mathcal{P}_{1^{\prime} 2^{\prime}} \mathcal{P}_{123} \sum_{s_{3}} \int L_{\boldsymbol{p}_{2} \boldsymbol{p}_{2}-\boldsymbol{k}_{3} \boldsymbol{k}_{3}}^{l_{2} l_{2}-s_{3} s_{3}}(2 \pi) q_{0}^{(2)-s_{3} s_{3}}\left(\boldsymbol{k}_{3}\right) q_{0}^{(2) l_{1} l_{2}}\left(\boldsymbol{p}_{2}\right) d \boldsymbol{k}_{3},
\end{aligned}
$$

with the short notation $\Omega_{k_{1} k_{2} k_{3} k_{4}}^{s_{2} s_{3} s_{4}}=s_{1} \omega\left(\boldsymbol{k}_{1}\right)+s_{2} \omega\left(\boldsymbol{k}_{2}\right)+s_{3} \omega\left(\boldsymbol{k}_{3}\right)+s_{4} \omega\left(\boldsymbol{k}_{4}\right)$.

We are interested in a solution of $q_{0}^{(2) l_{1} l_{2}}\left(\boldsymbol{p}_{2}\right)$ that remains bounded for times of order $O\left(\epsilon^{-2}\right)$. Then to capture the secular terms we take the limit $t \rightarrow \infty$ keeping $\epsilon^{2} t$ fixed, which really means that both $q_{0}^{(2) l_{1} l_{2}}\left(\boldsymbol{p}_{2}\right)$ and $F_{2}^{(2) l_{1} l_{2}}\left(\boldsymbol{p}_{2}\right)$ are constant over the 
integration in time. Such a procedure has an interpretation as an average. To see this, let us integrate over time, then divide both sides by $t$, and finally take the limit $t \rightarrow \infty$, keeping $\epsilon^{2} t$ fixed, yielding

$$
\begin{aligned}
F_{2}^{(2) l_{1} l_{2}}\left(\boldsymbol{p}_{2}\right) & =\lim _{t \rightarrow \infty} \frac{1}{t}\left\{-q_{2}^{(2) l_{1} l_{2}}\left(\boldsymbol{p}_{2}\right)+\alpha^{(2) l_{1} l_{2}}\left(\boldsymbol{p}_{2}\right)\right. \\
& +\mathcal{P}_{1^{\prime} 2^{\prime}} \sum_{s_{1} s_{2} s_{3}} \int L_{\boldsymbol{p}_{2} \boldsymbol{k}_{1} \boldsymbol{k}_{2} \boldsymbol{k}_{3}}^{l_{2} s_{1} s_{2} s_{3}} q_{0}^{(4) l_{1} s_{1} s_{2} s_{3}}\left(\boldsymbol{k}_{1}, \boldsymbol{k}_{2}, \boldsymbol{k}_{3}\right) \Delta\left(-\Omega_{k_{1} k_{2} k_{3} p_{2}}^{s_{1} s_{2} s_{3}-l_{2}}, t\right) \delta\left(\boldsymbol{p}_{1}+\boldsymbol{k}_{1}+\boldsymbol{k}_{2}+\boldsymbol{k}_{3}\right) d \boldsymbol{k}_{123} \\
& +\mathcal{P}_{1^{\prime} 2^{\prime}} \mathcal{P}_{123} \sum_{s_{2} s_{3}} \int L_{\boldsymbol{p}_{2} \boldsymbol{p}_{2}-\boldsymbol{k}_{3} \boldsymbol{k}_{3}}^{l_{2}-l_{2} s_{2} s_{3}}(2 \pi) q_{0}^{(2) s_{2} s_{3}}\left(\boldsymbol{k}_{3}\right) q_{0}^{(2) l_{1}-l_{2}}\left(\boldsymbol{p}_{2}\right) \Delta\left(-\Omega_{p_{2}-k_{3} k_{3} p_{2}}^{-l_{2} s_{2} s_{3}-l_{2}}, t\right) d \boldsymbol{k}_{3} \\
& +\mathcal{P}_{1^{\prime} 2^{\prime}} \mathcal{P}_{123} \sum_{s_{3}} \int L_{\boldsymbol{p}_{2} \boldsymbol{p}_{2}-\boldsymbol{k}_{3} \boldsymbol{k}_{3}}^{l_{2} l_{2} s_{3} s_{3}}(2 \pi) q_{0}^{(2) s_{3} s_{3}}\left(\boldsymbol{k}_{3}\right) q_{0}^{(2) l_{1} l_{2}}\left(\boldsymbol{p}_{2}\right) \Delta\left(-\Omega_{p_{2}-k_{3} k_{3} p_{2}}^{l_{2} s_{3} s_{3}-l_{2}}, t\right) d \boldsymbol{k}_{3} \\
& \left.+t\left(\mathcal{P}_{1^{\prime} 2^{\prime}} \mathcal{P}_{123} \sum_{s_{3}} \int L_{\boldsymbol{p}_{2} \boldsymbol{p}_{2}-\boldsymbol{k}_{3} \boldsymbol{k}_{3}}^{l_{2} l_{2}-s_{3} s_{3}}(2 \pi) q_{0}^{(2)-s_{3} s_{3}}\left(\boldsymbol{k}_{3}\right) q_{0}^{(2) l_{1} l_{2}}\left(\boldsymbol{p}_{2}\right) d \boldsymbol{k}_{3}\right)\right\}
\end{aligned}
$$

where $\alpha^{(2) l_{1} l_{2}}\left(\boldsymbol{p}_{2}\right)$ is an integration constant, that could depend on the slow varying scale time $\epsilon^{2} t$. In equation (123) we have defined the function

$$
\Delta(x, t) \equiv \int_{0}^{t} e^{i x \tau} d \tau=\frac{e^{i x t}-1}{i x}
$$

One sees that $F_{2}^{(2) l_{1} l_{2}}\left(\boldsymbol{p}_{2}\right)$ represents the time average over a time interval $t \sim \epsilon^{-2}$, then the slow dynamics is given by a long-time cumulative effect of the faster time dynamics. Because of the Riemann Lebesgue lemma ${ }^{7}$, if a function $f(x)$ is sufficiently smooth and decays sufficiently fast at $|x| \rightarrow \infty$ then [47]

$$
\lim _{t \rightarrow \infty} \int_{-\infty}^{\infty} f(x) \Delta(x, t) d x=\operatorname{sgn}(t) \pi f(0)+i P \int_{-\infty}^{\infty} \frac{f(x)}{x} d x,
$$

where $\mathrm{P}$ denotes the Cauchy principal value of the integral and $\operatorname{sgn}(t)=+1$ if $t>0$ and $\operatorname{sgn}(t)=-1$ if $t<0$. Symbolically, this result will be written as

$$
\lim _{t \rightarrow \infty} \Delta(x, t)=\operatorname{sgn}(t) \pi \delta(x)+i P\left(\frac{1}{x}\right) .
$$

As we suppose that $q_{0}^{(n) l_{1} \ldots l_{n}}\left(\boldsymbol{p}_{2}, \ldots, \boldsymbol{p}_{n}\right)$ is smooth at the initial time, we can use this last relation when taking the limit at $(123)^{8}$. Using also that $\alpha^{(2) l_{1} l_{2}}\left(\boldsymbol{p}_{2}\right)$ is constant and that $q_{2}^{(2) l_{1} l_{2}}\left(\boldsymbol{p}_{2}\right)$ must remain bounded to preserve a well orderer expansion, one gets the equation (45)

$$
F_{2}^{(2) l_{1} l_{2}}\left(\boldsymbol{p}_{2}\right)=3 \mathcal{P}_{1^{\prime} 2^{\prime}} \sum_{s} \int L_{\boldsymbol{p}_{2} \boldsymbol{p}_{2}-\boldsymbol{k} \boldsymbol{k}}^{l_{2} l_{2}-s s}(2 \pi) q_{0}^{(2)-s s}(\boldsymbol{k}) d \boldsymbol{k} q_{0}^{(2) l_{1} l_{2}}\left(\boldsymbol{p}_{2}\right),
$$

where we have used some symmetries to simplify the right hand side. The remainder terms of (123) give the next order correction of the second order cumulant:

$$
q_{2}^{(2) l_{1} l_{2}}\left(\boldsymbol{p}_{2}\right)=\alpha^{(2) l_{1} l_{2}}\left(\boldsymbol{p}_{2}\right)+\text { "non resonant regular terms". }
$$

In this case, the "non resonant regular terms" are precisely the non resonant terms in (123), that is, the terms that vanish as one take the limit of $1 / t$ times the right hand side of (123). They are regular in the sense of preserving the well ordered expansion in Fourier space, i.e. they do not have a singular behavior. The long time behavior of these terms, as we will see, can be always absorbed by the integration constant, thus they play no role in the slow dynamics.

\footnotetext{
${ }^{7}$ If the Lebesgue integral of $|f(x)|$ is finite, then $\lim _{t \rightarrow \pm \infty} \int_{-\infty}^{\infty} f(x) e^{i t x} d x \rightarrow 0$.

${ }^{8}$ It is also necessary to consider dispersive wave. If the frequency is proportional to the wave number, the last relation cannot be used in general. See for example [57, 58].
} 
10.4. The order $\epsilon^{2}$ correction for the fourth order cumulant.

Writing the equation for the fourth order cumulant (see equation (122)) of the Appendix 10.3) up to second order, using the change of variables (43) and the multi-scale expansion for time derivatives (42), the fourth order cumulant reads up to order $\epsilon^{2}$ :

$$
\begin{aligned}
& F_{2}^{(4) l_{1} l_{2} l_{3} l_{4}}\left(\boldsymbol{p}_{2}, \boldsymbol{p}_{3}, \boldsymbol{p}_{4}\right)=\lim _{t \rightarrow \infty} \frac{1}{t}\left\{-q_{2}^{(4) l_{1} l_{2} l_{3} l_{4}}\left(\boldsymbol{p}_{2}, \boldsymbol{p}_{3}, \boldsymbol{p}_{4}\right)+\alpha^{(4) l_{1} l_{2} l_{3} l_{4}}\left(\boldsymbol{p}_{2}, \boldsymbol{p}_{3}, \boldsymbol{p}_{4}\right)+\right. \\
& +\mathcal{P}_{1^{\prime} 2^{\prime} 3^{\prime} 4^{\prime}} \mathcal{P} \mathcal{T}_{123} \sum_{s_{1} s_{2} s_{3}} L_{\boldsymbol{p}_{4}-\boldsymbol{p}_{1}-\boldsymbol{p}_{2}-\boldsymbol{p}_{3}}^{l_{4} s_{1} s_{2} s_{3}}(2 \pi)^{2} q_{0}^{(2) s_{1} l_{1}}\left(\boldsymbol{p}_{1}\right) q_{0}^{(2) s_{2} l_{2}}\left(\boldsymbol{p}_{2}\right) q_{0}^{(2) s_{3} l_{3}}\left(\boldsymbol{p}_{3}\right) \Delta\left(-\Omega_{\left.p_{1} p_{2} p_{3} p_{3} p_{4} s_{4}, t\right)}^{s_{2} l_{4}}\right) \\
& \left.+t\left(\mathcal{P}_{1^{\prime} 2^{\prime} 3^{\prime} 4^{\prime}} \mathcal{P}_{123} \sum_{s_{2}} \int L_{\boldsymbol{p}_{4}-\boldsymbol{k}_{2} \boldsymbol{k}_{2} \boldsymbol{p}_{4}}^{l_{4}-s_{2} s_{2} l_{4}}(2 \pi) q_{0}^{(2)-s_{2} s_{2}}\left(\boldsymbol{k}_{2}\right) q_{0}^{(4) l_{1} l_{2} l_{3} l_{4}}\left(\boldsymbol{p}_{2}, \boldsymbol{p}_{3}, \boldsymbol{p}_{4}\right) d \boldsymbol{k}_{2}\right)\right\} \\
& +\quad \text { "non resonant regular terms" }
\end{aligned}
$$

and we can use a priori the same kind of arguments as before. However, the third term of (126)

$$
\mathcal{P}_{1^{\prime} 2^{\prime} 3^{\prime} 4^{\prime}} \mathcal{P} \mathcal{T}_{123} \sum_{s_{1} s_{2} s_{3}} L_{\boldsymbol{p}_{4}-\boldsymbol{p}_{1}-\boldsymbol{p}_{2}-\boldsymbol{p}_{3}}^{l_{4} s_{1} s_{2} s_{3}}(2 \pi)^{2} q_{0}^{(2) s_{1} l_{1}}\left(\boldsymbol{p}_{1}\right) q_{0}^{(2) s_{2} l_{2}}\left(\boldsymbol{p}_{2}\right) q_{0}^{(2) s_{3} l_{3}}\left(\boldsymbol{p}_{3}\right) \Delta\left(-\Omega_{p_{1} p_{2} p_{3} p_{4}}^{s_{1} s_{2} s_{3}-l_{4}}, t\right)
$$

deserves some caution because it does not have any integral over the $\boldsymbol{k}$-space. Therefore, it develop a non-smooth behavior at long times which is the main responsible of the long-time dynamics for $q_{0}^{(2) l_{1} l_{2}}\left(\boldsymbol{p}_{2}\right)$. These kind of terms are usually known as "live terms" [46, 47]. Although the expansion is no longer regular in the Fourier space, it is regular in the physical space (which is the pertinent one) since the transform into the real space requires some extra integrals of the cumulants. This extra integration is enough to ensure well defined functions, and therefore the asymptotic cumulant expansion remains valid. Even though there is no integral in the term (127), we can follow schematically the formal expression (124). The secular equation then leads to

$$
F_{2}^{(4) l_{1} l_{2} l_{3} l_{4}}\left(\boldsymbol{p}_{2}, \boldsymbol{p}_{3}, \boldsymbol{p}_{4}\right)=3 \mathcal{P}_{1^{\prime} 2^{\prime} 3^{\prime} 4^{\prime}}\left(\sum_{s} \int L_{\boldsymbol{p}_{4} \boldsymbol{p}_{4}-\boldsymbol{k} \boldsymbol{k}}^{l_{4} l_{4}-s s}(2 \pi) q_{0}^{(2)-s s}(\boldsymbol{k}) d \boldsymbol{k}\right) q_{0}^{(4) l_{1} l_{2} l_{3} l_{4}}\left(\boldsymbol{p}_{2}, \boldsymbol{p}_{3}, \boldsymbol{p}_{4}\right)
$$

and the first correction to the fourth order cumulant is

$$
\begin{aligned}
q_{2}^{(4) l_{1} l_{2} l_{3} l_{4}}\left(\boldsymbol{p}_{2}, \boldsymbol{p}_{3}, \boldsymbol{p}_{4}\right)=\alpha^{(4) l_{1} l_{2} l_{3} l_{4}}\left(\boldsymbol{p}_{2}, \boldsymbol{p}_{3}, \boldsymbol{p}_{4}\right)+\text { "non resonant regular terms" }+ \\
+\mathcal{P}_{1^{\prime} 2^{\prime} 3^{\prime} 4^{\prime}} \mathcal{P} \mathcal{T}_{123} \sum_{s_{1} s_{2} s_{3}} L_{\boldsymbol{p}_{4}-\boldsymbol{p}_{1}-\boldsymbol{p}_{2}-\boldsymbol{p}_{3}}^{l_{4} s_{1} s_{2} s_{3}}(2 \pi)^{2} q_{0}^{(2) s_{1} l_{1}}\left(\boldsymbol{p}_{1}\right) q_{0}^{(2) s_{2} l_{2}}\left(\boldsymbol{p}_{2}\right) q_{0}^{(2) s_{3} l_{3}}\left(\boldsymbol{p}_{3}\right) \Delta\left(-\Omega_{p_{1} p_{2} p_{3} s_{2} s_{2} s_{3}-l_{4}}^{s_{2}}, t\right)
\end{aligned}
$$

Note that $F_{2}^{(2)}$ and $F_{2}^{(4)}$ exhibit the same structure. Moreover, it is possible to show that for all order $n$

$$
F_{2}^{(n) l_{1} \ldots l_{n}}\left(\boldsymbol{p}_{2}, \ldots, \boldsymbol{p}_{n}\right)=3 \mathcal{P}_{1^{\prime} \ldots n^{\prime}}\left[\sum_{s} \int L_{\boldsymbol{p}_{n} \boldsymbol{p}_{n}-\boldsymbol{k} \boldsymbol{k}}^{l_{n} l_{n}-s s}(2 \pi) q_{0}^{(2)-s s}(\boldsymbol{k}) d \boldsymbol{k}\right] q_{0}^{(n) l_{1} \ldots l_{n}}\left(\boldsymbol{p}_{2}, \ldots, \boldsymbol{p}_{n}\right) .
$$

Then, one can integrate $q_{2}^{(n) l_{1} \ldots l_{n}}\left(\boldsymbol{p}_{2}, \ldots, \boldsymbol{p}_{n}\right)$ for all $n$. The constants of integration $\alpha_{2}^{(n) l_{1} \ldots l_{n}}\left(\boldsymbol{p}_{2}, \ldots, \boldsymbol{p}_{n}\right)$ play a relevant role in the expansion. Clearly these constants do depend on the slower time scales $\epsilon^{4} t$, and we can choose almost any value for $\alpha^{(n)}$ in such a way that they do not depend on the fast time scale. The only restriction it is that the cumulants $q^{(n)}$ must be smooth functions at $t=0$, that is, this choice cannot contradict the initial assumption. To avoid future complications we take $\alpha^{(n)}$ in order to have

$$
\lim _{t \rightarrow \infty} q_{2}^{(n) l_{1} \ldots l_{n}}\left(\boldsymbol{p}_{2}, \ldots, \boldsymbol{p}_{n}\right)=\lim _{t \rightarrow \infty} \quad \text { "live terms" }
$$

Explicitly this choice means that 


$$
\begin{aligned}
\lim _{t \rightarrow \infty} q_{2}^{(2) l_{1} l_{2}}\left(\boldsymbol{p}_{2}\right) & =0 \\
\lim _{t \rightarrow \infty} q_{2}^{(3) l_{1} l_{2} l_{3}}\left(\boldsymbol{p}_{2}, \boldsymbol{p}_{3}\right)= & 0 \\
\lim _{t \rightarrow \infty} q_{2}^{(4) l_{1} l_{2} l_{3} l_{4}}\left(\boldsymbol{p}_{2}, \boldsymbol{p}_{3}, \boldsymbol{p}_{4}\right)= & \mathcal{P}_{1^{\prime} 2^{\prime} 3^{\prime} 4^{\prime}} \mathcal{P} \mathcal{T}_{123} \sum_{s_{1} s_{2} s_{3}} L_{\boldsymbol{p}_{4}-\boldsymbol{p}_{1}-\boldsymbol{p}_{2}-\boldsymbol{p}_{3}}^{l_{4} s_{1} s_{2} s_{3}}(2 \pi)^{2} q_{0}^{(2) s_{1} l_{1}}\left(\boldsymbol{p}_{1}\right) q_{0}^{(2) s_{2} l_{2}}\left(\boldsymbol{p}_{2}\right) q_{0}^{(2) s_{3} l_{3}}\left(\boldsymbol{p}_{3}\right) \\
& \times\left(\operatorname{sgn}(t) \pi \delta\left(-\Omega_{p_{1} p_{2} p_{3} p_{4}}^{s_{1} s_{2} s_{3}-l_{4}}\right)+i P\left(\frac{1}{-\Omega_{p_{1} p_{2} p_{3} p_{3} s_{4}}^{s_{1} s_{3}-l_{4}}}\right)\right) .
\end{aligned}
$$

As we already mentioned, this choice is equivalent to absorb the long time behavior of the non resonant regular terms, hence:

$$
\alpha_{2}^{(n) l_{1} \ldots l_{n}}\left(\boldsymbol{p}_{2}, \ldots, \boldsymbol{p}_{n}\right)=\lim _{t \rightarrow \infty} \text { "non resonant regular terms". }
$$

At this order $\mathcal{O}\left(\epsilon^{2}\right)$, the resonant condition (46) gives only a nonlinear correction to the frequency. Moreover, from (45) and using the properties of the $L$ 's (32) one gets

$$
F_{2}^{(2)-l l}(\boldsymbol{p})=0,
$$

then $q_{0}^{(2)-l l}(\boldsymbol{p})$ does not depend on the slow time scale $\epsilon^{2} t$. For all the other cumulants one has an oscillatory dynamics

$$
q_{0}^{(n) l_{1} \ldots l_{n}}\left(\boldsymbol{p}_{2}, \ldots, \boldsymbol{p}_{n}\right)=\tilde{q}_{0}^{(n) l_{1} \ldots l_{n}}\left(\boldsymbol{p}_{2}, \ldots, \boldsymbol{p}_{n}\right) \exp \left(i \epsilon^{2} t\left(\mathcal{P}_{1^{\prime} \ldots n^{\prime}} \omega_{2}^{l_{1}}\left(\boldsymbol{p}_{1}\right)\right)\right)
$$

where $\omega_{2}^{l}(\boldsymbol{p})$ comes directly from (45), which is a second order correction to the dispersion relation:

$$
\omega_{2}^{l}(\boldsymbol{p})=-3 i \sum_{s} \int L_{\boldsymbol{p} \boldsymbol{p}-\boldsymbol{k} \boldsymbol{k}}^{l l-s s}(2 \pi) q_{0}^{(2)-s s}(\boldsymbol{k}) d \boldsymbol{k} .
$$

10.5. The order $\epsilon^{4}$ correction for the second order cumulant and closure.

As done in the $\frac{d}{d t} q_{0}^{(n)}$, in general, any higher order correction of the cumulant $q_{2}^{(n) l_{1} \ldots l_{n}}\left(\boldsymbol{p}_{2}, \ldots, \boldsymbol{p}_{n}\right)$ could depend on the slower time scales, so we can expand

$$
\frac{d}{d t} q_{2}^{(n) l_{1} \ldots l_{n}}\left(\boldsymbol{p}_{2}, \ldots, \boldsymbol{p}_{n}\right)=G_{0}^{(n) l_{1} \ldots l_{n}}\left(\boldsymbol{p}_{2}, \ldots, \boldsymbol{p}_{n}\right)+\epsilon^{2} G_{2}^{(n) l_{1} \ldots l_{n}}\left(\boldsymbol{p}_{2}, \ldots, \boldsymbol{p}_{n}\right) \ldots
$$

Doing the usual change of variable $Q_{4}^{(2) l_{1} l_{2}}\left(\boldsymbol{p}_{2}\right)=q_{4}^{(2) l_{1} l_{2}}\left(\boldsymbol{p}_{2}\right) \exp \left(-i\left(l_{1} \omega\left(\boldsymbol{p}_{1}\right)+l_{2} \omega\left(\boldsymbol{p}_{2}\right)\right) t\right)$, into the equation for the fourth order cumulant (see Appendix 10.2, equation (122)) one has the equation at order $\epsilon^{4}$ for the second order cumulant :

$$
\begin{aligned}
& F_{4}^{(2) l_{1} l_{2}}\left(\boldsymbol{p}_{2}\right)+G_{2}^{(2) l_{1} l_{2}}\left(\boldsymbol{p}_{2}\right)+\frac{d}{d t} q_{4}^{(2) l_{1} l_{2}}\left(\boldsymbol{p}_{2}\right)= \\
& \mathcal{P}_{1^{\prime} 2^{\prime}} \sum_{s_{1} s_{2} s_{3}} \int L_{\boldsymbol{p}_{2} \boldsymbol{k}_{1} \boldsymbol{k}_{2} \boldsymbol{k}_{3}}^{l_{2} s_{1} s_{2} s_{3}} q_{2}^{(4) l_{1} s_{1} s_{2} s_{3}}\left(\boldsymbol{k}_{1}, \boldsymbol{k}_{2}, \boldsymbol{k}_{3}\right) \delta\left(\boldsymbol{p}_{1}+\boldsymbol{k}_{1}+\boldsymbol{k}_{2}+\boldsymbol{k}_{3}\right) \exp \left(-i t \Omega_{p_{2} k_{1} k_{2} k_{3}}^{-l_{2} s_{1} s_{2} s_{3}}\right) d \boldsymbol{k}_{123}+ \\
& \mathcal{P}_{1^{\prime} 2^{\prime}{ }^{\prime}} \sum_{s_{1} s_{2} s_{3}} \int L_{\boldsymbol{p}_{2} \boldsymbol{p}_{2}-\boldsymbol{k}_{3} \boldsymbol{k}_{3}}^{l_{2} s_{1} s_{2} s_{3}} \mathcal{P}_{123}(2 \pi) q_{2}^{(2) l_{1} s_{1}}\left(\boldsymbol{p}_{2}\right) q_{0}^{(2) s_{2} s_{3}}\left(\boldsymbol{k}_{3}\right) \exp \left(-i t \Omega_{p_{2} p_{2} k_{3} k_{3}}^{-l_{2} s_{1} s_{2} s_{3}}\right) d \boldsymbol{k}_{3}+ \\
& \mathcal{P}_{1^{\prime} 2^{\prime}{ }^{\prime}} \sum_{s_{1} s_{2} s_{3}} \int L_{\boldsymbol{p}_{2} \boldsymbol{p}_{2}-\boldsymbol{k}_{3} \boldsymbol{k}_{3}}^{l_{2} s_{1} s_{2} s_{3}} \mathcal{P}_{123}(2 \pi) q_{0}^{(2) l_{1} s_{1}}\left(\boldsymbol{p}_{2}\right) q_{2}^{(2) s_{2} s_{3}}\left(\boldsymbol{k}_{3}\right) \exp \left(-i t \Omega_{p_{2} p_{1} k_{3} k_{3}}^{-l_{2} s_{1} s_{3} s_{3}}\right) d \boldsymbol{k}_{3}
\end{aligned}
$$

Replacing the cumulant $q_{2}^{(4) l_{1} s_{1} s_{2} s_{3}}\left(\boldsymbol{k}_{1}, \boldsymbol{k}_{2}, \boldsymbol{k}_{3}\right)$ given in (132) and integrating over the fast time scale, keeping $\epsilon^{4} t$ and $\epsilon^{2} t$ constant and following the same procedure used to solve the equation at order $\epsilon^{2}$, straightforward calculations give that the only possible 
relevant terms are

$$
\begin{aligned}
& F_{4}^{(2) l_{1} l_{2}}\left(\boldsymbol{p}_{2}\right)=\lim _{t \rightarrow \infty} \frac{1}{t}\left\{\mathcal{P}_{1^{\prime} 2^{\prime}} \mathcal{P} \mathcal{T}_{456} \sum_{s_{1} \cdots s_{6}} \int L_{\boldsymbol{p}_{2} \boldsymbol{k}_{1} \boldsymbol{k}_{2} \boldsymbol{k}_{3}}^{l_{2} s_{1} s_{2} s_{3}} L_{\boldsymbol{p}_{1}-\boldsymbol{k}_{1}-\boldsymbol{k}_{2}-\boldsymbol{k}_{3}}^{l_{1} s_{4} s_{5} s_{6}}(2 \pi)^{2} q_{0}^{(2) s_{4} s_{1}}\left(\boldsymbol{k}_{1}\right) q_{0}^{(2) s_{5} s_{2}}\left(\boldsymbol{k}_{2}\right) q_{0}^{(2) s_{6} s_{3}}\left(\boldsymbol{k}_{3}\right) \times\right. \\
& \times\left.\delta\left(\boldsymbol{p}_{1}+\boldsymbol{k}_{1}+\boldsymbol{k}_{2}+\boldsymbol{k}_{3}\right) \int_{0}^{t} \Delta\left(-\Omega_{\boldsymbol{k}_{1} \boldsymbol{k}_{2} \boldsymbol{k}_{3} \boldsymbol{p}_{1}}^{s_{4} s_{5} s_{6}-l_{1}}, \tau\right) \exp \left(-i \tau \Omega_{\boldsymbol{p}_{2} \boldsymbol{k}_{1} \boldsymbol{k}_{2} \boldsymbol{k}_{3}}^{-l_{2} s_{1} s_{2} s_{3}}\right) d \tau d \boldsymbol{k}_{123}\right\} \\
&+\lim _{t \rightarrow \infty} \frac{1}{t}\left\{\mathcal{P}_{1^{\prime} 2^{\prime}} \mathcal{P} \mathcal{T}_{456} \sum_{s_{1} \cdots s_{6}} \int L_{\boldsymbol{p}_{2} \boldsymbol{k}_{1} \boldsymbol{k}_{2} \boldsymbol{k}_{3}}^{l_{2} s_{1} s_{2} s_{3}} \mathcal{P}_{123} L_{\boldsymbol{k}_{3}-\boldsymbol{p}_{1}-\boldsymbol{k}_{1}-\boldsymbol{k}_{2}}^{s_{3} s_{4} s_{5} s_{6}}(2 \pi)^{2} q_{0}^{(2) s_{4} l_{1}}\left(\boldsymbol{p}_{1}\right) q_{0}^{(2) s_{5} s_{1}}\left(\boldsymbol{k}_{1}\right) q_{0}^{(2) s_{6} s_{2}}\left(\boldsymbol{k}_{2}\right) \times\right. \\
&\left.\times \delta\left(\boldsymbol{p}_{1}+\boldsymbol{k}_{1}+\boldsymbol{k}_{2}+\boldsymbol{k}_{3}\right) \int_{0}^{t} \Delta\left(-\Omega_{\boldsymbol{p}_{1} \boldsymbol{k}_{1} \boldsymbol{k}_{2} \boldsymbol{k}_{3}}^{s_{4} s_{5} s_{6}-s_{3}}, \tau\right) \exp \left(-i \tau \Omega_{\boldsymbol{p}_{2} \boldsymbol{k}_{1} \boldsymbol{k}_{2} \boldsymbol{k}_{3}}^{-l_{2} s_{1} s_{2} s_{3}}\right) d \tau d \boldsymbol{k}_{123}\right\}
\end{aligned}
$$

which reduce to the equations (47), (48) and (49) depending on the values of the indices $l_{1}$ and $l_{2}$.

10.6. Zakharov's transformation for the collisional integrals.

In this Appendix we precise the Zakharov's transformations to obtain simplified formulas for both collisional integrals $\mathrm{Coll}_{3 \leftrightarrow 1}[n](68)$ and $\mathrm{Coll}_{2 \leftrightarrow 2}(69)$ of Section 6.1. As we mention in the text the case of the collisional term $C o l l_{3 \leftrightarrow 1}[n]$ will be realized in detail.

The key idea is to find a change of variables over the second term (77)

$$
12 \pi K^{3} \int_{\mathcal{D}} S_{\omega_{p} \omega_{1} \omega_{2} \omega_{3}}\left(\omega_{1} \omega_{2} \omega_{3} \omega_{p}\right)^{-x}\left(\omega_{p}^{x}-\omega_{1}^{x}+\omega_{2}^{x}+\omega_{3}^{x}\right) \delta\left(\omega_{1}-\omega_{p}-\omega_{2}-\omega_{3}\right) d \omega_{123},
$$

that maps the line $\omega_{1}-\omega_{p}-\omega_{2}-\omega_{3}=0$ into the line $\omega_{p}-\omega_{1}-\omega_{2}-\omega_{3}=0$. The transformation reads

$$
\omega_{1}=\frac{\omega_{p}^{2}}{\tilde{\omega}_{1}}, \quad \omega_{2}=\frac{\omega_{p} \tilde{\omega}_{2}}{\tilde{\omega}_{1}}, \quad \omega_{3}=\frac{\omega_{p} \tilde{\omega}_{3}}{\tilde{\omega}_{1}},
$$

that has a Jacobian $\left|\frac{D\left(\omega_{1}, \omega_{2}, \omega_{3}\right)}{D\left(\tilde{\omega}_{1}, \tilde{\omega}_{2}, \tilde{\omega}_{3}\right)}\right|=\left(\frac{\omega_{p}}{\omega_{1}}\right)^{4}$. Re-arranging terms one has

$$
12 \pi K^{3} \int_{O} S_{\omega_{p} \frac{\omega_{p}^{2}}{\tilde{\omega}_{1}} \frac{\omega_{p} \tilde{\omega}_{2}}{\tilde{\omega}_{1}} \frac{\omega_{p} \tilde{\omega}_{3}}{\tilde{\omega}_{1}}}\left(\omega_{p} \tilde{\omega}_{1} \tilde{\omega}_{2} \tilde{\omega}_{3}\right)^{-x}\left(\tilde{\omega}_{1}^{x}-\omega_{p}^{x}+\tilde{\omega}_{2}^{x}+\tilde{\omega}_{3}^{x}\right)\left(\frac{\omega_{p}}{\tilde{\omega}_{1}}\right)^{-3 x} \delta\left(\frac{\omega_{p}}{\tilde{\omega}_{1}}\left(\omega_{p}-\tilde{\omega}_{1}-\tilde{\omega}_{2}-\tilde{\omega}_{3}\right)\right) \mid \frac{D\left(\omega_{1}, \omega_{2}, \omega_{3}\right)\left|d\left(\tilde{\omega}_{1}, \tilde{\omega}_{2}, \tilde{\omega}_{3}\right)\right|}{d \tilde{\omega}_{123}},
$$

where the new domain of integration, after the change of variables, is exactly the region $O$, in Fig. 2.

Replacing the Jacobian and because of the degree of homogeneity of the $S$-matrix (66)

$$
S_{\omega_{p} \frac{\omega_{p}^{2}}{\tilde{\omega}_{1}} \frac{\omega_{p} \tilde{\omega}_{2}}{\tilde{\omega}_{1}} \frac{\omega_{p} \tilde{\omega}_{3}}{\tilde{\omega}_{1}}}=\left(\frac{\tilde{\omega}_{1}}{\omega_{p}}\right) S_{\omega_{1} \omega_{p} \omega_{2} \omega_{3}}=\left(\frac{\tilde{\omega}_{1}}{\omega_{p}}\right) S_{\omega_{1} \omega_{p} \omega_{2} \omega_{3}}
$$

one finally has

$$
-12 \pi K^{3} \int_{O} S_{\tilde{\omega}_{1} \omega_{p} \tilde{\omega}_{2} \tilde{\omega}_{3}}\left(\omega_{p} \tilde{\omega}_{1} \tilde{\omega}_{2} \tilde{\omega}_{3}\right)^{-x}\left(\omega_{p}^{x}-\tilde{\omega}_{1}^{x}-\tilde{\omega}_{2}^{x}-\tilde{\omega}_{3}^{x}\right)\left(\frac{\omega_{p}}{\tilde{\omega}_{1}}\right)^{-3 x+2} \delta\left(\omega_{p}-\tilde{\omega}_{1}-\tilde{\omega}_{2}-\tilde{\omega}_{3}\right) d \tilde{\omega}_{123} .
$$

The same procedure may be done for the other two terms in (68) exchanging $1 \leftrightarrow 2$ and $1 \leftrightarrow 3$, therefore one gets at the end that

$$
\begin{aligned}
\operatorname{Coll}_{3 \leftrightarrow 1}\left[K \omega_{p}^{-x}\right]= & 12 \pi K^{3} \int_{O} S_{\omega_{p} \omega_{1} \omega_{2} \omega_{3}}\left(\omega_{p} \omega_{1} \omega_{2} \omega_{3}\right)^{-x}\left(\omega_{p}^{x}-\omega_{1}^{x}-\omega_{2}^{x}-\omega_{3}^{x}\right) \\
& \times\left(1-\left(\frac{\omega_{p}}{\omega_{1}}\right)^{-3 x+2}-\left(\frac{\omega_{p}}{\omega_{2}}\right)^{-3 x+2}-\left(\frac{\omega_{p}}{\omega_{3}}\right)^{-3 x+2}\right) \delta\left(\omega_{p}-\omega_{1}-\omega_{2}-\omega_{3}\right) d \omega_{123}
\end{aligned}
$$

Rewriting everything in terms of dimensionless variables of integration $u_{i}=\frac{\omega_{i}}{\omega_{p}}$ one gets equation (78) and (79). 
Similarly, the collisional term $\mathrm{Coll}_{2 \leftrightarrow 2}$, maybe written after an integration of (69) with respect to $\omega_{1}$ yielding

$$
\operatorname{Coll}_{2 \leftrightarrow 2}[n]=36 \pi K^{3} \int_{\mathcal{D}} S_{\omega_{p} \omega_{1} \omega_{2} \omega_{3}} n_{1} n_{2} n_{3} n_{p}\left(\frac{1}{n_{p}}+\frac{1}{n_{1}}-\frac{1}{n_{2}}-\frac{1}{n_{3}}\right) d \omega_{23},
$$

where $\omega_{1}=\omega_{2}+\omega_{3}-\omega_{p}$. Because $\omega_{1} \geq 0$ one has that the domain of integration exclude the region $O$ in Fig. 2 , that is $\mathcal{D}=I \cup I I \cup I I I \cup I V\left(\omega_{2} \geq 0, \omega_{3} \geq 0\right.$ and $\left.\omega_{2}+\omega_{3}-\omega_{p} \geq 0\right)$. This domain of integration may be divided in the four distinct domains: $I, I I, I I I \& I V$ (see Fig. 2).

As in the previous collisional term $\operatorname{Coll}_{3 \leftrightarrow 1}[n]$, one looks for a power law solution of the form $n_{p}=K \omega_{p}^{-x}$, then one splits the integral in the four different domains $I, I I, I I I \& I V$ and finally the domains $I I, I I I \& I V$ are mapped into $I$ via the following transformations :

$$
\begin{gathered}
I I \rightarrow I: \quad \omega_{2}=\frac{\omega_{p} \tilde{\omega}_{3}}{\tilde{\omega}_{1}}, \quad \omega_{3}=\frac{\omega_{p} \tilde{\omega}_{2}}{\tilde{\omega}_{1}}, \Rightarrow \omega_{1}=\frac{\omega_{p}^{2}}{\tilde{\omega}_{1}} \\
I I I \rightarrow I: \quad \omega_{2}=\frac{\omega_{p} \tilde{\omega}_{1}}{\tilde{\omega}_{3}}, \quad \omega_{3}=\frac{\omega_{p}^{2}}{\tilde{\omega}_{3}} \Rightarrow \omega_{1}=\frac{\omega_{p} \tilde{\omega}_{2}}{\tilde{\omega}_{3}} \\
I V \rightarrow I: \quad \omega_{2}=\frac{\omega_{p}^{2}}{\tilde{\omega}_{2}} \quad \omega_{3}=\frac{\omega_{p} \tilde{\omega}_{1}}{\tilde{\omega}_{2}}, \quad \Rightarrow \quad \omega_{1}=\frac{\omega_{p} \tilde{\omega}_{3}}{\tilde{\omega}_{2}},
\end{gathered}
$$

where $\tilde{\omega}_{1}=\tilde{\omega}_{2}+\tilde{\omega}_{3}-\omega_{p}$. The final collision integral, which is defined over the domain $I$ of Fig. 2 , reads

$$
\begin{aligned}
\operatorname{Coll}_{2 \leftrightarrow 2}[n] & =36 \pi K^{3} \int_{I} S_{\omega_{p} \omega_{1} \omega_{2} \omega_{3}}\left(\omega_{p} \omega_{1} \omega_{2} \omega_{3}\right)^{-x}\left(\omega_{p}^{x}+\omega_{1}^{x}-\omega_{2}^{x}-\omega_{3}^{x}\right) \\
& \times\left(1+\left(\frac{\omega_{p}}{\omega_{1}}\right)^{-3 x+2}-\left(\frac{\omega_{p}}{\omega_{2}}\right)^{-3 x+2}-\left(\frac{\omega_{p}}{\omega_{3}}\right)^{-3 x+2}\right) \delta\left(\omega_{p}+\omega_{1}-\omega_{2}-\omega_{3}\right) d \omega_{123}
\end{aligned}
$$

where the tilde over the $\omega$ 's were removed and the $\delta$-function was reintroduced to show explicitly the energy conservation of the wave interaction. Notice that the integration domain $I$ (defined in Fig. 2) differs from the one in the $3 \leftrightarrow 1$ interaction. As previously one may rewrite (138) using dimensionless variable $u_{i}=\frac{\omega_{i}}{\omega_{p}}$ getting equations (80) and (81).

\subsection{Bounds of the S-Matrix and the modified $\hat{S}$-Matrix.}

The exact calculation of the isotropic scattering matrix (64)

$$
S_{\omega_{p} \omega_{1} \omega_{2} \omega_{3}}=\frac{p}{\omega_{p}^{\prime}} \frac{k_{1}}{\omega_{1}^{\prime}} \frac{k_{2}}{\omega_{2}^{\prime}} \frac{k_{3}}{\omega_{3}^{\prime}} \int_{0}^{2 \pi}\left|J_{p \boldsymbol{k}_{1}, \boldsymbol{k}_{2} \boldsymbol{k}_{3}}\right|^{2} \delta\left(\boldsymbol{p}+\boldsymbol{k}_{1}+\boldsymbol{k}_{2}+\boldsymbol{k}_{3}\right) d \varphi_{p} d \varphi_{k_{1}} d \varphi_{k_{2}} d \varphi_{k_{3}} .
$$

with

$$
\begin{aligned}
\left|J_{\boldsymbol{p} \boldsymbol{k}_{1}, \boldsymbol{k}_{2} \boldsymbol{k}_{3}}\right|^{2}= & \frac{1}{9}\left(X_{\boldsymbol{p}} X_{\boldsymbol{k}_{1}} X_{\boldsymbol{k}_{2}} X_{\boldsymbol{k}_{3}}\right)^{2}\left[\left|T_{\boldsymbol{p} \boldsymbol{k}_{1} ; \boldsymbol{k}_{2} \boldsymbol{k}_{3}}\right|^{2}+\left|T_{\boldsymbol{p} \boldsymbol{k}_{2} ; \boldsymbol{k}_{1} \boldsymbol{k}_{3}}\right|^{2}+\left|T_{\boldsymbol{p} \boldsymbol{k}_{3} ; \boldsymbol{k}_{2} \boldsymbol{k}_{1}}\right|^{2}+\right. \\
& \left.2 T_{\boldsymbol{p} \boldsymbol{k}_{1} ; \boldsymbol{k}_{2} \boldsymbol{k}_{3}} T_{\boldsymbol{p} \boldsymbol{k}_{2} ; \boldsymbol{k}_{1} \boldsymbol{k}_{3}}+2 T_{\boldsymbol{p} \boldsymbol{k}_{1} ; \boldsymbol{k}_{2} \boldsymbol{k}_{3}} T_{\boldsymbol{p} \boldsymbol{k}_{3} ; \boldsymbol{k}_{2} \boldsymbol{k}_{1}}+2 T_{\boldsymbol{p} \boldsymbol{k}_{2} ; \boldsymbol{k}_{1} \boldsymbol{k}_{3}} T_{\boldsymbol{p} \boldsymbol{k}_{3} ; \boldsymbol{k}_{2} \boldsymbol{k}_{1}}\right] .
\end{aligned}
$$

requires the calculation of two types of integrals. Namely the perfect square: $\left|T_{\boldsymbol{p} \boldsymbol{k}_{1}, \boldsymbol{k}_{2} ; \boldsymbol{k}_{3}}\right|^{2}$, and the cross term: $2 T_{\boldsymbol{p} \boldsymbol{k}_{1} ; \boldsymbol{k}_{2} \boldsymbol{k}_{3}} T_{\boldsymbol{p} \boldsymbol{k}_{2} ; \boldsymbol{k}_{1} \boldsymbol{k}_{3}}$. The integration of the cross term is a complicate problem, fortunately to study the convergence of the collisional term we do not need to calculate them. A simple lower and upper bound for the expression (140) can be obtained

$$
\frac{1}{9}\left[\left|T_{\boldsymbol{p} \boldsymbol{k}_{1}, \boldsymbol{k}_{2} ; \boldsymbol{k}_{3}}\right|^{2}+\left|T_{\boldsymbol{p} \boldsymbol{k}_{2} ; \boldsymbol{k}_{1} \boldsymbol{k}_{3}}\right|^{2}+\left|T_{\boldsymbol{p} \boldsymbol{k}_{3} ; \boldsymbol{k}_{2} \boldsymbol{k}_{1}}\right|^{2}\right] \leq \frac{\left|J_{\boldsymbol{p} \boldsymbol{k}_{1} \boldsymbol{k}_{2} \boldsymbol{k}_{3}}\right|^{2}}{\left(X_{\boldsymbol{p}} X_{\boldsymbol{k}_{1}} X_{\boldsymbol{k}_{2}} X_{\boldsymbol{k}_{3}}\right)^{2}} \leq \frac{1}{3}\left[\left|T_{\boldsymbol{p} \boldsymbol{k}_{1}, \boldsymbol{k}_{2} ; \boldsymbol{k}_{3}}\right|^{2}+\left|T_{\boldsymbol{p} \boldsymbol{k}_{2} ; \boldsymbol{k}_{1} \boldsymbol{k}_{3}}\right|^{2}+\left|T_{\boldsymbol{p} \boldsymbol{k}_{3} ; \boldsymbol{k}_{2} \boldsymbol{k}_{1}}\right|^{2}\right] .
$$

The l.h.s of the inequality follows directly from (31) and the positiveness of (26), and the r.h.s inequality follows from the Cauchy-Schwarz inequality. Therefore, the $S$-matrix (139) can also be bounded as

$$
\frac{1}{3} \hat{S}_{\omega_{p} \omega_{1} \omega_{2} \omega_{3} \omega} \leq S_{\omega_{p} \omega_{1} \omega_{2} \omega_{3}} \leq \hat{S}_{\omega_{p} \omega_{1} \omega_{2} \omega_{3}}
$$


where the modified $S$-matrix

$$
\hat{S}_{\omega_{p} \omega_{1} \omega_{2} \omega_{3}}=\frac{1}{3} \frac{p}{\omega_{p}^{\prime}} \frac{k_{1}}{\omega_{1}^{\prime}} \frac{k_{2}}{\omega_{2}^{\prime}} \frac{k_{3}}{\omega_{3}^{\prime}}\left(X_{\boldsymbol{p}} X_{\boldsymbol{k}_{1}} X_{\boldsymbol{k}_{2}} X_{\boldsymbol{k}_{3}}\right)^{2}\left[I_{p k_{1} k_{2} k_{3}}+I_{p k_{2} k_{1} k_{3}}+I_{p k_{3} k_{2} k_{1}}\right],
$$

and

$$
I_{p k_{1} k_{2} k_{3}}=\int_{0}^{2 \pi}\left|T_{\boldsymbol{p} \boldsymbol{k}_{1} ; \boldsymbol{k}_{2} \boldsymbol{k}_{3}}\right|^{2} \delta\left(\boldsymbol{p}+\boldsymbol{k}_{1}+\boldsymbol{k}_{2}+\boldsymbol{k}_{3}\right) d \varphi_{k} d \varphi_{k_{1}} d \varphi_{k_{2}} d \varphi_{k_{3}},
$$

which correspond to the perfect square term in eq. (140).

We shall add an extra integral $\delta\left(\boldsymbol{p}+\boldsymbol{k}_{1}+\boldsymbol{k}_{2}+\boldsymbol{k}_{3}\right)=\int d \boldsymbol{\lambda} \delta\left(\boldsymbol{p}+\boldsymbol{k}_{1}+\boldsymbol{\lambda}\right) \delta\left(\boldsymbol{k}_{2}+\boldsymbol{k}_{3}-\boldsymbol{\lambda}\right)$. The order of the indices $\boldsymbol{p}, \boldsymbol{k}_{1}, \ldots$ are chosen depending on the indices of the corresponding term $\left|T_{\boldsymbol{p} \boldsymbol{k}_{1} ; \boldsymbol{k}_{2} \boldsymbol{k}_{3}}\right|^{2}$. Therefore, equation (143) becomes

$$
I_{p k_{1} k_{2} k_{3}}=\int d \boldsymbol{\lambda} \int_{0}^{2 \pi}\left|T_{\boldsymbol{p} \boldsymbol{k}_{1} ; \boldsymbol{k}_{2} \boldsymbol{k}_{3}}\right|^{2} \delta\left(\boldsymbol{p}+\boldsymbol{k}_{1}+\boldsymbol{\lambda}\right) \delta\left(\boldsymbol{k}_{2}+\boldsymbol{k}_{3}-\boldsymbol{\lambda}\right) d \varphi_{k} d \varphi_{k_{1}} d \varphi_{k_{2}} d \varphi_{k_{3}} .
$$

The last integral would be over $\boldsymbol{\lambda}$, so that for the integrations with respect to $\varphi_{k}, \varphi_{1}, \varphi_{2}, \varphi_{3}, \in[0,2 \pi]$ one assumes that $\lambda=(\lambda, 0)$.

$$
\begin{aligned}
I_{k k_{1} k_{2} k_{3}=}= & \int d \boldsymbol{\lambda} \int_{0}^{2 \pi}\left|T_{\boldsymbol{p} \boldsymbol{k}_{1} ; \boldsymbol{k}_{2} \boldsymbol{k}_{3}}\right|^{2} \delta\left(k \cos \varphi_{k}+k_{1} \cos \varphi_{1}+\lambda\right) \delta\left(k \sin \varphi_{k}+k_{1} \sin \varphi_{1}\right) \times \\
& \times \delta\left(k_{2} \cos \varphi_{2}+k_{3} \cos \varphi_{3}+\lambda\right) \delta\left(k_{2} \sin \varphi_{2}+k_{3} \sin \varphi_{3}\right) d \varphi_{k} d \varphi_{1} d \varphi_{2} d \varphi_{3} .
\end{aligned}
$$

The whole expression can be simplified because

$$
\int_{0}^{2 \pi} f\left(\varphi_{k}, \varphi_{1}\right) \delta\left(k \cos \varphi_{k}+k_{1} \cos \varphi_{1}+\lambda\right) \delta\left(k \sin \varphi_{k}+k_{1} \sin \varphi_{1}\right) d \varphi_{k} d \varphi_{1}=\frac{f\left(\varphi_{k}^{*}, \varphi_{1}^{*}\right)}{2 S_{p k_{1} \boldsymbol{\lambda}}}
$$

where

$$
S_{\boldsymbol{p} \boldsymbol{k}_{1} \boldsymbol{\lambda}}=\frac{1}{4} \sqrt{\left(\left(k+k_{1}\right)^{2}-\lambda^{2}\right)\left(\lambda^{2}-\left(k-k_{1}\right)^{2}\right)},
$$

is the area of the triangle built with the triad vectors $\boldsymbol{p}, \boldsymbol{k}_{1}, \boldsymbol{\lambda}$, and $\varphi_{k}^{*}, \varphi_{1}^{*}$ are the roots that vanish the arguments of the $\delta$-functions. Finally, because the tensor $T_{\boldsymbol{p} \boldsymbol{k}_{1} ; \boldsymbol{k}_{2} \boldsymbol{k}_{3}}$ of (26) depends explicitly on $1 /\left|\boldsymbol{p}+\boldsymbol{k}_{1}\right|^{4}=1 / \lambda^{4}$ and $\left|\boldsymbol{p} \times \boldsymbol{k}_{1}\right|=2 S_{\boldsymbol{p} \boldsymbol{k}_{1} \boldsymbol{\lambda}}$, one gets

$$
I_{p k_{1} k_{2} k_{3}}=2 \pi \int_{\lambda_{\min }}^{\lambda_{\max }} \frac{\lambda d \lambda}{\lambda^{8}}\left(S_{\boldsymbol{p} \boldsymbol{k}_{1} \boldsymbol{\lambda}}\right)^{3}\left(S_{\boldsymbol{k}_{2} \boldsymbol{k}_{3} \boldsymbol{\lambda}}\right)^{3}
$$

where

$$
\lambda_{\min }=\max \left\{\left|k-k_{1}\right|,\left|k_{2}-k_{3}\right|\right\} \quad \text { and } \quad \lambda_{\max }=\min \left\{k+k_{1}, k_{2}+k_{3}\right\} .
$$

Rewriting the latter expression in term of the frequencies after the convenient change of variable $u=\lambda^{2} / 2$ one gets

$$
\left.I_{\omega_{p} \omega_{1} ; \omega_{2} \omega_{3}}=\frac{\pi}{2^{9}} \int_{u_{\min }}^{u_{\max }} \Psi\left(\left(\sqrt{\omega_{p}}+\sqrt{\omega_{1}}\right)^{2},\left(\sqrt{\omega_{p}}-\sqrt{\omega_{1}}\right)^{2} ;\left(\sqrt{\omega_{2}}+\sqrt{\omega_{3}}\right)^{2},\left(\sqrt{\omega_{2}}-\sqrt{\omega_{3}}\right)^{2}\right) ; u\right) d u
$$

where we define

$$
\Psi(a, b ; c, d ; u)=\frac{1}{u^{4}}[(a-u)(u-b)]^{3 / 2}[(c-u)(u-d)]^{3 / 2}
$$

and the integration limits reads

$$
u_{\min }=\max \left\{\left(\sqrt{\omega_{p}}-\sqrt{\omega_{1}}\right)^{2},\left(\sqrt{\omega_{2}}-\sqrt{\omega_{3}}\right)^{2}\right\} \quad \text { and } \quad u_{\max }=\min \left\{\left(\sqrt{\omega_{p}}+\sqrt{\omega_{1}}\right)^{2},\left(\sqrt{\omega_{2}}+\sqrt{\omega_{3}}\right)^{2}\right\} .
$$


10.8. Appendix. Sigular behavior of the $\hat{S}$-matrix in the $\omega_{2}, \omega_{3}$ plane.

In this appendix we shall study the convergence of the collisional integrals

$$
\hat{I}_{2 \leftrightarrow 2}^{(1)}(x)=36 \pi \int_{I} \hat{S}_{1 u_{1} u_{2} u_{3}} \operatorname{coll}^{(+)} d u_{23} \quad \text { and } \quad \hat{I}_{3 \leftrightarrow 1}^{(1)}(x)=12 \pi \int_{O} \hat{S}_{1 u_{1} u_{2} u_{3}} \operatorname{coll}^{(-)} d u_{23},
$$

where

$$
\operatorname{coll}^{(s)}=\left(u_{2} u_{3} u_{1}\right)^{-x}\left(1+s u_{1}^{x}-u_{2}^{x}-u_{3}^{x}\right)\left(1+s u_{1}^{3 x-2}-u_{2}^{3 x-2}-u_{3}^{3 x-2}\right) .
$$

For the $2 \leftrightarrow 2$ processes $u_{1}=\left(u_{2}+u_{3}-1\right)$ and for the $3 \leftrightarrow 1$ processes $u_{1}=\left(1-u_{2}-u_{3}\right)$.

As shown in Fig. 3 the $\hat{S}$-matrix presents various singularities in the $u_{2}-u_{3}$ plane. These singularities are located near the lines $u_{2}+u_{3}=1, u_{2}=1$ and $u_{3}=1$, and also near the three points $\left(u_{2}=1, u_{3}=0,\left(u_{2}=0, u_{3}=1\right)\right.$, and $\left(u_{2}=1, u_{3}=1\right)$. To determine the convergence of the integral we need to determine the dominant behavior of the integrand near the critical zones. Let us first consider the integral $\hat{I}_{2 \leftrightarrow 2}^{(1)}$ over the domain I in Fig. 2. In this domain there are various critical zones which are located near the lines $u_{2}+u_{3}=1, u_{2}=1$ and $u_{3}=1$, and also near the three corners of the triangles $u_{2}=1, u_{3}=0, u_{2}=0$, $u_{3}=1$, and $u_{2}=1, u_{3}=1$. We shall consider them separately.

10.8.1. The line $u_{2}+u_{3}=1$

In this case we take $u_{1}=\epsilon \delta u_{1}$ (with $\epsilon \ll 1$ ), and the other two variables may be written in terms of a new one $\Omega=u_{2}-u_{3}$. In this way, $u_{2}$ and $u_{3}$ take the form:

$$
u_{2}=\frac{1}{2}\left(1+\epsilon \delta u_{1}+\Omega\right) \quad \text { and } \quad u_{3}=\frac{1}{2}\left(1+\epsilon \delta u_{1}-\Omega\right) .
$$

We need to evaluate the three integrals of (83). The first contribution reads:

$$
I_{1, \epsilon \delta u_{1} ; u_{2}, u_{3}}=\int_{\left(1-\sqrt{\epsilon \delta u_{1}}\right)^{2}}^{\left(1+\sqrt{\epsilon \delta u_{1}}\right)^{2}} \psi(u) d u
$$

where

$$
\psi(u)=\Psi\left(\left(1+\sqrt{\epsilon \delta u_{1}}\right)^{2},\left(1-\sqrt{\epsilon \delta u_{1}}\right)^{2} ;\left(1+\epsilon \delta u_{1}\right)+\sqrt{\left(1+\epsilon \delta u_{1}\right)^{2}-\Omega^{2}},\left(1+\epsilon \delta u_{1}\right)-\sqrt{\left(1+\epsilon \delta u_{1}\right)^{2}-\Omega^{2}} ; u\right)
$$

and $\Psi(a, b, c, d, u)$ was defined in Eq. (146). The key point here, is that the integral is concentrated around 1. Therefore it can be approximated by

$$
I_{1, \epsilon \delta u_{1} ; u_{2}, u_{3}}=4 \sqrt{\epsilon \delta u_{1}} \psi(1) \approx 32 \epsilon^{2} \delta u_{1}^{2}\left(1-\Omega^{2}\right)^{3 / 2} .
$$

The next contribution comes from

$$
I_{1, u_{2} ; \epsilon \delta u_{1}, u_{3}}=\int_{u_{\min }}^{u_{\max }} \psi(u) d u
$$

where

$$
\psi(u) \approx \Psi\left((1+\sqrt{(1+\Omega) / 2})^{2},(1-\sqrt{(1+\Omega) / 2})^{2} ;(1+\Omega) / 2,(1+\Omega) / 2 ; u\right) .
$$

In the limit $\epsilon \rightarrow 0$, one has that the previous integral is concentrate over a very small region

$$
\begin{aligned}
& u_{\min }=\left(-\sqrt{\epsilon \delta u_{1}}+\sqrt{\left(1+\epsilon \delta u_{1}-\Omega\right) / 2}\right)^{2} \approx \frac{1}{2}(1-\Omega)-\epsilon^{1 / 2} \sqrt{2(1-\Omega) \delta u_{1}} \\
& u_{\max }=\left(\sqrt{\epsilon \delta u_{1}}+\sqrt{\left(1+\epsilon \delta u_{1}-\Omega\right) / 2}\right)^{2} \approx \frac{1}{2}(1-\Omega)+\epsilon^{1 / 2} \sqrt{2(1-\Omega) \delta u_{1}}
\end{aligned}
$$

Therefore,

$$
I_{1, u_{2} ; \epsilon \delta u_{1}, u_{3}} \approx \int_{u_{\min }}^{u_{\max }} \psi(u) d u \approx 2 \epsilon^{1 / 2} \sqrt{2(1-\Omega) \delta u_{1}} \psi((1-\Omega) / 2) \approx 128 \epsilon^{2} \delta u_{1}^{2} \frac{(1+\Omega)^{3 / 2}}{(1-\Omega)^{1 / 2}}
$$


A similar result arises for $I_{1, u_{3} ; u 2, \epsilon \delta u_{1}}$ changing $\Omega \rightarrow-\Omega$. Therefore, the final $\hat{S}$ matrix behaves up to a leading order to:

$$
\hat{S}_{1, \epsilon \delta u_{1} ; u_{2}, u_{3}} \approx \frac{\pi}{12} \epsilon \delta u_{1} \frac{\left(9+6 \Omega^{2}+8 \Omega^{4}\right)}{\left(1-\Omega^{2}\right)^{3 / 2}}+\mathcal{O}\left(\epsilon^{2}\right) .
$$

From the singular behavior of (147), one gets that the collisional integrand has two dominant behaviors depending on the value of $x$ :

$$
\operatorname{coll}^{(+)}\left(\delta u_{1}, \Omega\right) \sim\left\{\begin{array}{cc}
\left(\epsilon \delta u_{1}\right)^{2 x-2} f_{1}(\Omega, x) & x<2 / 3 \\
\left(\epsilon \delta u_{1}\right)^{-x} f_{2}(\Omega, x) & x>2 / 3
\end{array}\right.
$$

where $f_{1 / 2}(\Omega, x)$ are two functions of $\Omega$, that we omit for simplicity.

Therefore, we can deduce that the collisional integral in the strip $u_{1} \in[0, \epsilon]$ reads,

$$
\hat{I}_{2 \leftrightarrow 2}^{(1)} \sim \epsilon \int_{0}^{\epsilon} \delta u_{1} d \delta u_{1} \int_{-1+\epsilon \delta u_{1}}^{1-\epsilon \delta u_{1}} \hat{S}\left(\delta u_{1}, \Omega\right) \operatorname{coll}^{(+)}\left(\delta u_{1}, \Omega\right) d \Omega \sim\left\{\begin{array}{cc}
\epsilon^{2 x} & x<2 / 3 \\
\epsilon^{2-x} & x>2 / 3
\end{array} .\right.
$$

Therefore, this integral converges, for any small $\epsilon$, if $1 / 2<x<2$.

10.8.2. The line $u_{2}=1$.

We shall approximate de $\hat{S}$-matrix in the line $u_{2} \approx 1$ and $u_{3} \in[0,1]$. In this case we take $u_{2}=1+\epsilon \delta u_{2}$, hence $u_{1}=u_{3}+\epsilon \delta u_{2}$. First, we compute the contribution of

$$
I_{1, u_{3}+\epsilon \delta u_{2} ; 1+\epsilon \delta u_{2}, u_{3}}=\frac{\pi}{512} \int_{\left(1-\sqrt{u_{3}+\epsilon \delta u_{2}}\right)^{2}}^{\left(1+\sqrt{u_{3}+\epsilon \delta u_{2}}\right)^{2}} \psi(u) d u
$$

where

$$
\psi(u)=\Psi\left(\left(1+\sqrt{u_{3}+\epsilon \delta u_{2}}\right)^{2},\left(1-\sqrt{u_{3}+\epsilon \delta u_{2}}\right)^{2} ;\left(\sqrt{u_{3}}+\sqrt{1+\epsilon \delta u_{2}}\right)^{2},\left(\sqrt{u_{3}}-\sqrt{1+\epsilon \delta u_{2}}\right)^{2} ; u\right)
$$

In this case, it is easy to see that the final result would be at order $\epsilon^{0}$, we approximate the integrand by

$$
\psi(u)=\frac{\pi}{512 u^{4}}\left(-u^{2}+2 u\left(u_{3}+1\right)-\left(u_{3}-1\right)^{2}\right)^{3}+\mathcal{O}(\epsilon)
$$

hence, the final result is

$$
I_{1, u_{3}+\epsilon \delta u_{2} ; 1+\epsilon \delta u_{2}, u_{3}} \approx \frac{\pi}{384}\left(-4 \sqrt{u_{3}}\left(15 u_{3}^{2}+14 u_{3}+15\right)+6\left(1+u_{3}\right)\left(5 u_{3}^{2}-2 u_{3}+5\right) \log \left(\frac{1+\sqrt{u_{3}}}{1-\sqrt{u_{3}}}\right)\right)+\mathcal{O}(\epsilon)
$$

Next, we consider the integral

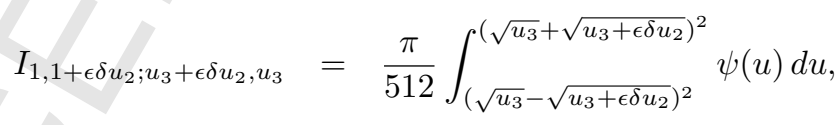

where

$$
\psi(u)=\Psi\left(\left(1+\sqrt{1+\epsilon \delta u_{2}}\right)^{2},\left(1-\sqrt{1+\epsilon \delta u_{2}}\right)^{2} ;\left(\sqrt{u_{3}}+\sqrt{u_{3}+\epsilon \delta u_{2}}\right)^{2},\left(\sqrt{u_{3}}-\sqrt{u_{3}+\epsilon \delta u_{2}}\right)^{2} ; u\right)
$$

Because the lower limit $\left(\sqrt{u_{3}}-\sqrt{u_{3}+\epsilon \delta u_{2}}\right)^{2} \approx \epsilon^{2} \frac{\delta u_{2}^{2}}{4 u_{3}}$ the integral diverges as $u \rightarrow 0$. Keeping, the singular behavior of $\psi(u)$ near $u \approx 0$, we approximate $\psi(u)$ by:

$$
\psi(u) \approx \Psi\left(4,\left(1-\sqrt{1+\epsilon \delta u_{2}}\right)^{2} ; 4 u_{3},\left(\sqrt{u_{3}}-\sqrt{u_{3}+\epsilon \delta u_{2}}\right)^{2} ; u\right) .
$$


Keeping the singular behavior of order $\log \epsilon$ one gets

$$
I_{1,1+\epsilon \delta u_{2} ; u_{3}+\epsilon \delta u_{2}, u_{3}} \approx-\frac{\pi}{8} u_{3}^{3 / 2} \log \left(\epsilon^{2} \delta u_{2}^{2}\right)+\mathcal{O}(1)
$$

The third term, leads also to a regular behavior, therefore the only singular behavior comes from equation (150). The final $\hat{S}$-matrix diverges logarithmically as

$$
\hat{S}_{1 u_{1} u_{2} u_{3}} \approx-\frac{\pi}{24 u_{3}^{1 / 2}} \log \left(\epsilon^{2} \delta u_{2}^{2}\right)+\mathcal{O}(1)
$$

On the other hand the wave interaction term behaves as

$$
\operatorname{coll}^{(+)} \approx \epsilon^{2} x(3 x-2) u_{3}^{-2(2+x)}\left(u_{3}-u_{3}^{x}\right)^{2}\left(u_{3}^{2}+u_{3}^{2 x}+u_{3}^{1+x}\right) \delta u_{2}^{2}+\mathcal{O}\left(\epsilon^{3}\right) .
$$

The final collisional integral scales as:

$$
\hat{I}_{2 \leftrightarrow 2}^{(1)}=\int_{\epsilon}^{1} d u_{3} \int_{-1}^{0}\left(\epsilon d \delta u_{2}\right) \hat{S}_{1 u_{1} u_{2} u_{3}} \operatorname{coll}^{(+)} d u_{2} \sim \frac{\pi}{18} x(3 x-2)\left(\frac{\epsilon^{7 / 2-2 x}}{1-4 x}+\frac{\epsilon^{5 / 2-x}}{1+2 x}+\frac{\epsilon^{x+1 / 2}}{5-2 x}+\frac{\epsilon^{2 x-1 / 2}}{4 x-7}\right) \log (\epsilon) .
$$

Therefore, the integral $\hat{I}_{2 \leftrightarrow 2}^{(1)}$ is bounded for any $1 / 4<x<7 / 4$ at this singular point.

10.8.3. The point $u_{2}=1$ and $u_{3}=0$.

Next, we investigate the situation around one of the corners of the integration domain $u_{2} \approx 1$ and $u_{3} \approx 0$. More precisely, we take $u_{2}=1+\epsilon \delta u_{2}, u_{3}=\epsilon \delta u_{3}$, hence $u_{1}=\epsilon \delta u_{1}=\epsilon\left(\delta u_{2}+\delta u_{3}\right)$.

First, we compute the contribution of

$$
I_{1, \epsilon \delta u_{1} ; 1+\epsilon \delta u_{2}, \epsilon \delta u_{3}}=\int_{\left(1-\sqrt{\epsilon \delta u_{1}}\right)^{2}}^{\left(1+\sqrt{\epsilon \delta u_{1}}\right)^{2}} \psi(u) d u
$$

where

$$
\psi(u)=\Psi\left(\left(1+\sqrt{\epsilon \delta u_{1}}\right)^{2},\left(1-\sqrt{\epsilon \delta u_{1}}\right)^{2} ;\left(\sqrt{1+\epsilon \delta u_{2}}+\sqrt{\epsilon \delta u_{3}}\right)^{2},\left(\sqrt{1+\epsilon \delta u_{2}}-\sqrt{\epsilon \delta u_{3}}\right)^{2} ; u\right) .
$$

In the limit $\epsilon \rightarrow 0$, one gets

$$
I_{1, \epsilon \delta u_{1} ; 1+\epsilon \delta u_{2}, \epsilon \delta u_{3}} \approx 4 \sqrt{\epsilon \delta u_{1}} \psi(1)+\text { h.o.t. } \approx \frac{\pi}{2} \delta u_{1}^{2} \delta u_{3}^{3 / 2} \epsilon^{7 / 2}+\text { h.o.t }
$$

Next contribution reads

$$
I_{1,1+\epsilon \delta u_{2} ; \epsilon \delta u_{1}, \epsilon \delta u_{3}}=\int_{\epsilon\left(\sqrt{\delta u_{1}}-\sqrt{\delta u_{3}}\right)^{2}}^{\epsilon\left(\sqrt{\delta u_{1}}+\sqrt{\delta u_{3}}\right)^{2}} \psi(u) d u
$$

where

$$
\begin{aligned}
\psi(u) & =\Psi\left(\left(1+\sqrt{1+\epsilon \delta u_{2}}\right)^{2},\left(1-\sqrt{1+\epsilon \delta u_{2}}\right)^{2} ; \epsilon\left(\sqrt{\delta u_{1}}+\sqrt{\delta u_{3}}\right)^{2}, \epsilon\left(\sqrt{\delta u_{1}}-\sqrt{\delta u_{3}}\right)^{2} ; u\right) \\
& \approx \frac{8}{u^{5 / 2}}\left[\left(\epsilon\left(\sqrt{\delta u_{1}}+\sqrt{\delta u_{3}}\right)^{2}-u\right)\left(u-\epsilon\left(\sqrt{\delta u_{1}}-\sqrt{\delta u_{3}}\right)^{2}\right)\right]^{3 / 2}\left[4 \epsilon\left(\delta u_{1}+\delta u_{3}\right)\right]^{3 / 2} .
\end{aligned}
$$

In the second approximation we have neglected a $\epsilon^{2} \delta u_{2}^{2}$ factor because the leading order is for $u \sim \epsilon$. This readily gives the final result up to order $\epsilon^{3 / 2}$. Moreover, the numerical pre-factors posses a close form:

$$
I_{1,1+\epsilon \delta u_{2} ; \epsilon \delta u_{1}, \epsilon \delta u_{3}} \approx \epsilon^{3 / 2} \frac{\pi}{12}\left(\delta u_{1} \delta u_{3}\right)^{1 / 4}\left(4\left(\delta u_{1}+\delta u_{3}\right) E[\varphi, m]-\left(2 \delta u_{1}+2 \delta u_{3}-\sqrt{\delta u_{1} \delta u_{3}}\right) F[\varphi, m]\right)
$$


where $F(\varphi \mid m)$ and $E(\varphi \mid m)$ are the elliptic integrals of first kind $F$ and second kind $E$ respectively, and the parameters read

$$
\varphi=\arcsin \left(2 \frac{\left(\delta u_{1} \delta u_{3}\right)^{1 / 4}}{\sqrt{\delta u_{1}}+\sqrt{\delta u_{3}}}\right) \quad \& \quad m=\frac{\left(\sqrt{\delta u_{1}}+\sqrt{\delta u_{3}}\right)^{2}}{4 \sqrt{\delta u_{1} \delta u_{3}}}
$$

Finally, the expression

$$
I_{1, \epsilon \delta u_{3} ; 1+\epsilon \delta u_{2}, \epsilon \delta u_{1}}=\frac{\pi}{2} \epsilon^{7 / 2} \delta u_{3}^{2} \delta u_{1}^{3 / 2}
$$

by symmetry arguments with the first term. Therefore, the final behavior of the $\hat{S}$-matrix is up to its dominant order as $\epsilon^{-1 / 2}$ :

$$
\hat{S}_{1 u_{1} u_{2} u_{3}} \approx \frac{\pi}{36 \epsilon^{1 / 2}}\left(\delta u_{1} \delta u_{3}\right)^{-3 / 4}\left(4\left(\delta u_{1}+\delta u_{3}\right) E[\varphi, m]-\left(2 \delta u_{1}+2 \delta u_{3}-\sqrt{\delta u_{1} \delta u_{3}}\right) F[\varphi, m]\right) .
$$

The collisional interaction term diverges as:

$$
\operatorname{coll}^{(+)} \approx\left\{\begin{array}{cc}
\epsilon^{2 x-2}\left(\delta u_{3}^{-x}-\delta u_{1}^{-x}\right)\left(\delta u_{1}^{3 x-2}-\delta u_{1}^{3 x-2}\right) & x<1 \\
x(3 x-2) \epsilon^{2-2 x} \delta u_{2}^{2} \delta u_{1}^{-x} \delta u_{3}^{-x} & x>1
\end{array} .\right.
$$

The convergence of the integral is therefore conditioned by the integral (notice the change of variables $\int_{0}^{\epsilon} d u_{3} \int_{1-u_{3}}^{1} d u_{2} \rightarrow$ $\left.\epsilon^{2} \int_{0}^{1} d \delta u_{3} \int_{-\delta u_{3}}^{0} d \delta u_{2}\right)$

$$
\hat{I}_{2 \leftrightarrow 2}^{(1)} \approx \epsilon^{2} \int_{0}^{1} d \delta u_{3} \int_{-\delta u_{3}}^{0} \int_{1-u_{3}}^{1} \hat{S}_{1 u_{1} u_{2} u_{3}} \operatorname{coll}^{(+)} d \delta u_{2} \sim\left\{\begin{array}{cc}
\epsilon^{2 x-1 / 2} & x<1 \\
\epsilon^{7 / 2-2 x} & x>1
\end{array}\right.
$$

Therefore the collisional integral converges, for all $\epsilon>0$, if $1 / 4<x<7 / 4$.

10.8.4. The point $u_{2}=1$ and $u_{3}=1$.

Near this singular point the one has $u_{2}=1+\epsilon \delta u_{2}, u_{3}=1+\epsilon \delta u_{3}$, hence $u_{1}=1+\epsilon \delta u_{1}$ with $\delta u_{1}=\left(\delta u_{2}+\delta u_{3}\right)$. All these infinitesimal being negative.

First, we compute the contribution of

$$
I_{1,1+\epsilon \delta u_{1} ; 1+\epsilon \delta u_{2}, 1+\epsilon \delta u_{3}}=\int_{\left(1-\sqrt{1+\epsilon \delta u_{1}}\right)^{2}}^{\left(1+\sqrt{1+\epsilon \delta u_{1}}\right)^{2}} \psi(u) d u
$$

where

$$
\begin{aligned}
\psi(u) & =\Psi\left(\left(1+\sqrt{1+\epsilon \delta u_{1}}\right)^{2},\left(1-\sqrt{1+\epsilon \delta u_{1}}\right)^{2} ;\left(\sqrt{1+\epsilon \delta u_{2}}+\sqrt{1+\epsilon \delta u_{3}}\right)^{2},\left(\sqrt{1+\epsilon \delta u_{2}}-\sqrt{1+\epsilon \delta u_{3}}\right)^{2} ; u\right)(15 \\
& \approx \Psi\left(4, \frac{\epsilon^{2}}{4} \delta u_{1}^{2} ; 4, \frac{\epsilon^{2}}{4}\left(\delta u_{2}-\delta u_{3}\right)^{2} ; u\right)
\end{aligned}
$$

In the limit $\epsilon \rightarrow 0$, one gets integrating and expanding up to leading order in $\epsilon$ one gets:

$$
I_{1,1+\epsilon \delta u_{1} ; 1+\epsilon \delta u_{2}, 1+\epsilon \delta u_{3}} \approx-64\left(\frac{\left(\delta u_{2}^{2}+\delta u_{3}^{2}\right)\left(\delta u_{2}^{4}-4 \delta u_{2}^{2} \delta u_{3}^{2}+\delta u_{3}^{4}\right)}{\left(\delta u_{2}^{2}-\delta u_{3}^{2}\right)^{3}} \log \left(\frac{\delta u_{2}}{\delta u_{3}}\right)+\log \left(\epsilon^{2} \delta u_{2} \delta u_{3}\right)\right)+\mathcal{O}\left(\epsilon^{0}\right) .
$$

Similarly, other terms in (83) are obtained by exchanging $\delta u_{1}$ by $\delta u_{2}$ and $\delta u_{3}$ in the general integral (155).

In the first case,

$$
\psi(u) \approx \Psi\left(4, \frac{\epsilon^{2}}{4} \delta u_{2}^{2} ; 4, \frac{\epsilon^{2}}{4}\left(\delta u_{1}-\delta u_{3}\right)^{2} ; u\right)
$$

but $\delta u_{1}-\delta u_{3}=\delta u_{2}$ therefore 


$$
I_{1,1+\epsilon \delta u_{2} ; 1+\epsilon \delta u_{1}, 1+\epsilon \delta u_{3}} \approx-64 \log \left(\epsilon^{2} \delta u_{2}^{2}\right)+\mathcal{O}\left(\epsilon^{0}\right) .
$$

And the same results holds for the remaining integral but changing $\delta u_{2}^{2} \rightarrow \delta u_{3}^{2}$.

Therefore the final $\hat{S}$-matrix is

$$
\hat{S}_{1 u_{1} u_{2} u_{3}} \approx-\frac{\pi}{24}\left(\frac{\left(\delta u_{2}^{2}+\delta u_{3}^{2}\right)\left(\delta u_{2}^{4}-4 \delta u_{2}^{2} \delta u_{3}^{2}+\delta u_{3}^{4}\right)}{\left(\delta u_{2}^{2}-\delta u_{3}^{2}\right)^{3}} \log \left(\frac{\delta u_{2}}{\delta u_{3}}\right)+3 \log \left(\epsilon^{2} \delta u_{2} \delta u_{3}\right)\right) .
$$

On the other hand the collisional term behaves as

$$
\text { coll }=3 x(x-1)^{2}(3 x-2) \epsilon^{4} \delta u_{2}^{2} \delta u_{3}^{2}+\mathcal{O}\left(\epsilon^{5}\right)
$$

The convergence of the integral is therefore conditioned by the integral (notice the change of variables $\int_{1-\epsilon}^{1} \int_{1-\epsilon}^{1} d u_{3} d u_{2} \rightarrow$ $\left.\epsilon^{2} \int_{-1}^{0} \int_{-1}^{0} d \delta u_{3} d \delta u_{2}\right)$

$$
\hat{I}_{2 \leftrightarrow 2}^{(1)} \approx \epsilon^{2} \int_{-1}^{0} \int_{-1}^{0} d \delta u_{3} d \delta u_{2} \hat{S}_{1 u_{1} u_{2} u_{3}} \operatorname{coll}(+) d \delta u_{2} \sim \epsilon^{6}
$$

Therefore the collisional integral converges, for all $\epsilon>0$.

10.8.5. The point $u_{2}=0$ and $u_{3}=0$.

In this section we focus on the $3 \leftrightarrow 1$ collisional integral (79). Contrary to the case $2 \leftrightarrow 2$, in the present situation there are four critical regions inside the domain O in Fig. 2. The three already discussed and because $\hat{S}_{1 u_{1} u_{2} u_{3}}$ is a continuous function, the convergence criteria is the same. Therefore, we shall focus in the fourth singular point at the origin $u_{2}=0$ and $u_{3}=0$.

In this last case, we take $u_{2}=\epsilon \delta u_{2}, u_{3}=\epsilon \delta u_{3}$, but $u_{1}=1-\epsilon \delta u_{1}$. (Here $\delta u_{1}=\delta u_{2}+\delta u_{3}$.)

$$
I_{1,1-\epsilon \delta u_{1} ; \epsilon \delta u_{2}, \epsilon \delta u_{3}}=\int_{u_{\min }}^{u_{\max }} \psi(u) d u
$$

where

$$
\psi(u)=\Psi\left(\left(1+\sqrt{1-\epsilon \delta u_{1}}\right)^{2},\left(1-\sqrt{1-\epsilon \delta u_{1}}\right)^{2} ; \epsilon\left(\sqrt{\delta u_{2}}+\sqrt{\delta u_{3}}\right)^{2}, \epsilon\left(\sqrt{\delta u_{2}}-\sqrt{\delta u_{3}}\right)^{2} ; u\right)
$$

where the limits are defined after $(84)$. There are two cases, the first domain is defined near the diagonal $\delta u_{2} \approx \delta u_{3}$ while the second domain is defined through $\epsilon\left(\sqrt{\delta u_{2}}-\sqrt{\delta u_{3}}\right)^{2} \geq \epsilon^{2} \delta u_{1}^{2} / 4$.

In the later case,

$$
I_{1,1-\epsilon \delta u_{1} ; \epsilon \delta u_{2}, \epsilon \delta u_{3}}=\int_{\epsilon\left(\sqrt{\delta u_{2}}-\sqrt{\delta u_{3}}\right)^{2}}^{\epsilon\left(\sqrt{\delta u_{2}}+\sqrt{\delta u_{3}}\right)^{2}} \psi(u) d u
$$

and keeping in mind that the $u \sim \epsilon$ we approximate

$$
\psi(u) \approx \frac{8}{u^{5 / 2}}\left(u-\epsilon\left(\sqrt{\delta u_{2}}-\sqrt{\delta u_{3}}\right)^{2}\right)^{3 / 2}\left(\epsilon\left(\sqrt{\delta u_{2}}+\sqrt{\delta u_{3}}\right)^{2}-u\right)^{3 / 2}
$$

noticing that the final scaling should be $\epsilon^{3 / 2}$. The final result becomes:

$$
I_{1,1-\epsilon\left(\delta u_{2}+\delta u_{3}\right) ; \epsilon \delta u_{2}, \epsilon \delta u_{3}} \approx \epsilon^{3 / 2} \frac{\pi}{12}\left(\delta u_{2} \delta u_{3}\right)^{1 / 4}\left[\left(2\left(\delta u_{2}+\delta u_{3}\right)-\sqrt{\delta u_{2} \delta u_{3}}\right) F(\varphi \mid m)-4\left(\delta u_{2}+\delta u_{3}\right) E(\varphi \mid m)\right]
$$

with

$$
\varphi=\sin ^{-1}\left(\frac{2\left(\delta u_{2} \delta u_{3}\right)^{1 / 4}}{\sqrt{\delta u_{2}}+\sqrt{\delta u_{3}}}\right) \text { and } m=\frac{\left(\sqrt{\delta u_{2}}+\sqrt{\delta u_{3}}\right)^{2}}{4 \sqrt{\delta u_{2} \delta u_{3}}} .
$$


The final, interaction matrix becomes

$$
\hat{S}_{1,1-\epsilon\left(\delta u_{2}+\delta u_{3}\right) ; \epsilon \delta u_{2}, \epsilon \delta u_{3}} \approx \epsilon^{-1 / 2} \frac{\pi}{36\left(\delta u_{2} \delta u_{3}\right)^{3 / 4}}\left[\left(2\left(\delta u_{2}+\delta u_{3}\right)-\sqrt{\delta u_{2} \delta u_{3}}\right) F(\varphi \mid m)-4\left(\delta u_{2}+\delta u_{3}\right) E(\varphi \mid m)\right],
$$

if $\epsilon\left(\sqrt{\delta u_{2}}-\sqrt{\delta u_{3}}\right)^{2} \geq \epsilon^{2} \delta u_{1}^{2} / 4$. change.

On the other hand if $\epsilon\left(\sqrt{\delta u_{2}}-\sqrt{\delta u_{3}}\right)^{2} \leq \epsilon^{2} \delta u_{1}^{2} / 4$, one needs to re-compute the integrals because the integration domains

$$
I_{1,1-2 \epsilon \delta u_{2} ; \epsilon \delta u_{2}, \epsilon \delta u_{2}} \approx \int_{\left(1-\sqrt{1-2 \epsilon \delta u_{2}}\right)^{2}}^{4 \epsilon \delta u_{2}} \psi(u) d u
$$

where

$$
\psi(u) \approx \frac{8}{u^{5 / 2}}\left(u-4 \epsilon \delta u_{2}\right)^{3 / 2}\left(\left(1-\sqrt{1-2 \epsilon \delta u_{2}}\right)^{2}-u\right)^{3 / 2} .
$$

Because both limits are near the origin, the final result maybe computed using the same kind of elliptic integrals, the final behavior reads,

$$
I_{1,1-2 \epsilon \delta u_{2} ; \epsilon \delta u_{2}, \epsilon \delta u_{2}} \approx-\frac{\pi}{8} \epsilon^{3 / 2} \delta u_{2}^{3 / 2} \log \left(\epsilon \delta u_{2}\right)+\text { h.o.t. }
$$

Therefore, in this case, the $\hat{S}$ matrix is approximated by

$$
\hat{S}_{1 u_{1} u_{2} u_{2}}=\frac{\pi}{24} \epsilon^{3 / 2} \delta u_{2}^{3 / 2} \log \left(\epsilon \delta u_{2}\right) .
$$

if $\epsilon\left(\sqrt{\delta u_{2}}-\sqrt{\delta u_{3}}\right)^{2} \leq \epsilon^{2} \delta u_{1}^{2} / 4$.

On the other hand, the four waves interaction term (147) is approximated there by

$$
\operatorname{coll}^{(-)} \approx\left\{\begin{array}{cc}
\epsilon^{2 x-2}\left(\delta u_{2}^{-x}+\delta u_{3}^{-x}\right)\left(\delta u_{2}^{3 x-2}+\delta u_{3}^{3 x-2}\right) & x<1 \\
x(3 x-2) \epsilon^{2-2 x} \delta u_{1}^{2} \delta u_{2}^{-x} \delta u_{3}^{-x} & x>1
\end{array} .\right.
$$

The convergence of the integral is therefore conditioned by the integral (notice the change of variables $\int_{0}^{\epsilon} \int_{0}^{\epsilon} d u_{3} d u_{2} \rightarrow$ $\left.\epsilon^{2} \int_{0}^{1} \int_{0}^{1} d \delta u_{2} d \delta u_{3}\right)$

$$
\hat{I}_{2 \leftrightarrow 2}^{(1)} \approx \epsilon^{2} \int_{0}^{1} \int_{0}^{1} \hat{S}_{1 u_{1} u_{2} u_{3}} \operatorname{coll}^{(-)} d \delta u_{2} d \delta u_{3} \sim\left\{\begin{array}{cc}
\epsilon^{2 x-1 / 2} & x<1 \\
\epsilon^{7 / 2-2 x} & x>1
\end{array} .\right.
$$

Therefore, the collisional integral converges, for all $\epsilon>0$, if $1 / 4<x<7 / 4$.

\subsection{Logarithmic correction of the Kolmogorov-Zakharov spectrum}

When the KZ spectrum coincides with the equilibrium solution, no power law solution seems to exist. However, one may pursue a perturbative expansion to catch a stationary, non power law constant flux solution. Because of the degeneracy aspect of the solution one seeks an expansion of the form

$$
n_{\omega}=K \omega^{-x} \log ^{z}\left(\omega / \omega_{c}\right) .
$$

We Introduce this Ansatz into the collisional terms and apply the change of variables $\omega_{i}=\omega u_{i}$. Because of the locality of interactions one may expand the collisional integral in the parameter $\left|\log \left(\omega / \omega_{c}\right)\right| \gg\left|\log u_{i}\right|$. The result is a series of the form

$$
\operatorname{Coll}_{3 \leftrightarrow 1}[n]=K^{3} \omega^{1-3 x}\left(I_{3 \leftrightarrow 1}^{(1)}(x) \log ^{3 z}\left(\omega / \omega_{c}\right)+z I_{3 \leftrightarrow 1}^{(2)}(x) \log ^{3 z-1}\left(\omega / \omega_{c}\right)+I_{3 \leftrightarrow 1}^{(3)}(x, z) \log ^{3 z-2}\left(\omega / \omega_{c}\right)+\ldots\right),
$$

similarly

$$
\operatorname{Coll}_{2 \leftrightarrow 2}[n]=K^{3} \omega^{1-3 x}\left(I_{2 \leftrightarrow 2}^{(1)}(x) \log ^{3 z}\left(\omega / \omega_{c}\right)+z I_{2 \leftrightarrow 2}^{(2)}(x) \log ^{3 z-1}\left(\omega / \omega_{c}\right)+I_{2 \leftrightarrow 2}^{(3)}(x, z) \log ^{3 z-2}\left(\omega / \omega_{c}\right)+\ldots\right),
$$


where we have neglected terms of order $O\left(\log ^{3 z-3}\left(\omega / \omega_{c}\right)\right)$ and smaller. The integrals $I_{3 \leftrightarrow 1}^{(1)}(x)$ and $I_{2 \leftrightarrow 2}^{(1)}(x)$ are exactly $(79)$ and (81) respectively (with $\gamma=-1$ ). Other integrals are cumbersome so that we do not write them here. Because both expansions have the same form we shall define $I^{(1)}(x) \equiv I_{3 \leftrightarrow 1}^{(1)}(x)+I_{2 \leftrightarrow 2}^{(1)}(x)$ and similarly for others, $I^{(2)}(x)$ and $I^{(3)}(x, z)$. As before, replacing into the equation for the energy $P(75)$, one gets

$$
\begin{aligned}
P=-\int_{0}^{\omega} K^{3} \omega^{2-3 x}\left(I^{(1)}(x) \log ^{3 z}\left(\omega / \omega_{c}\right)+z I^{(2)}(x) \log ^{3 z-1}\left(\omega / \omega_{c}\right)+I^{(3)}(x) \log ^{3 z-2}\left(\omega / \omega_{c}\right)+\ldots\right) d \omega \\
=K^{3} \omega_{c}^{3(1-x)}\left[I^{(1)}(x) \frac{1}{3^{3 z+1}(x-1)^{3 z+1}} \Gamma\left(3 z+1,3(x-1) \log \left(\omega / \omega_{c}\right)\right)\right. \\
+z I^{(2)}(x) \frac{1}{3^{3 z}(x-1)^{3 z}} \Gamma\left(3 z, 3(x-1) \log \left(\omega / \omega_{c}\right)\right) \\
\left.\quad+I^{(3)}(x, z) \frac{1}{3^{3 z-1}(x-1)^{3 z-1}} \Gamma\left(3 z-1,3(x-1) \log \left(\omega / \omega_{c}\right)\right)+\ldots\right]
\end{aligned}
$$

Here we have used the integral

$$
\int_{0}^{\omega} \omega^{2-3 x} \log ^{s}\left(\omega / \omega_{c}\right) d \omega=-\frac{\omega_{c}^{3(1-x)}}{3^{1+s}(x-1)^{1+s}} \Gamma\left(1+s, 3(x-1) \log \left(\omega / \omega_{c}\right)\right),
$$

where $\Gamma(1+s, z)=\int_{z}^{\infty} t^{s} e^{-t} d t$ is the incomplete $\Gamma$-function.

Now we study the local behavior of (160) around $x \approx 1$. Expanding the required quantities around $x=1$, up to the lowest non-trivial order one gets

$$
\begin{aligned}
I^{(1)}(x) & \approx \frac{1}{2} I^{(1)^{\prime \prime}}(1)(x-1)^{2}+\mathcal{O}(x-1)^{3} \\
I^{(2)}(x) & \approx I^{(2)^{\prime}}(1)(x-1)+\mathcal{O}(x-1)^{2} \\
I^{(3)}(x, z) & \approx I^{(3)}(1, z)+\mathcal{O}(x-1) .
\end{aligned}
$$

Where the required expressions are quite simple

$$
\begin{aligned}
\lim _{x \rightarrow 1^{-}} I^{(1)^{\prime \prime}}(x)= & 72 \pi\left(\int_{O} S_{1 u_{1} u_{2} u_{3}}\left(u_{1} u_{2} u_{3}\right)^{-1}\left(u_{1} \log u_{1}+u_{2} \log u_{2}+u_{3} \log u_{3}\right)^{2} \delta\left(1-u_{1}-u_{2}-u_{3}\right) d u_{123}+\right. \\
& \left.3 \int_{I} S_{1 u_{1} u_{2} u_{3}}\left(u_{1} u_{2} u_{3}\right)^{-1}\left(-u_{1} \log u_{1}+u_{2} \log u_{2}+u_{3} \log u_{3}\right)^{2} \delta\left(1+u_{1}-u_{2}-u_{3}\right) d u_{123}\right) \\
\lim _{x \rightarrow 1^{-}} I^{(2)^{\prime}}(x)= & -I^{(1)^{\prime \prime}}(1), \\
\lim _{x \rightarrow 1^{-}} I^{(3)}(x, z)= & \frac{z(3 z-1)}{6} I^{(1)^{\prime \prime}}(1) .
\end{aligned}
$$

Finally, doing the same kind of expansions for the $\Gamma$ one has

$$
\begin{aligned}
\Gamma\left(3 z+1,3(x-1) \log \left(\omega / \omega_{c}\right)\right) & \approx \Gamma(3 z+1)+\mathcal{O}(x-1) \\
\Gamma\left(3 z, 3(x-1) \log \left(\omega / \omega_{c}\right)\right) & \approx \Gamma(3 z)+\mathcal{O}(x-1) \\
\Gamma\left(3 z-1,3(x-1) \log \left(\omega / \omega_{c}\right)\right) & \approx \frac{1}{3 z-1}\left(\Gamma(3 z)-\left(3(x-1) \log \left(\omega / \omega_{c}\right)\right)^{3 z-1} e^{-3(x-1) \log \left(\omega / \omega_{c}\right)}\right)+O(x-1) .
\end{aligned}
$$

Therefore

$$
\begin{aligned}
P= & K^{3} I^{(1)^{\prime \prime}}(1) \frac{\omega_{c}^{3(1-x)}}{(x-1)^{3 z-1}}\left[\frac{\Gamma(3 z+1)}{23^{3 z+1}}-z \frac{\Gamma(3 z)}{3^{3 z}}+\frac{z}{63^{3 z-1}} \Gamma(3 z)\right]+ \\
& -K^{3} I^{(1)^{\prime \prime}}(1) \omega_{c}^{3(1-x)}\left[\frac{z}{63^{3 z-1}}\left(3 \log \left(\omega / \omega_{c}\right)\right)^{3 z-1} e^{-3(x-1) \log \left(\omega / \omega_{c}\right)}\right] .
\end{aligned}
$$


We have not taken any kind of limit so far. Expression (163) is only an expansion up to first non trivial order in $(x-1)$. Naturally, the first term of (163) is independent of $\omega$, representing thus a constant flux. The second term does depend explicitly on $\omega$ but it becomes independent of $\omega$ in the limit $x \rightarrow 1$. This limit, on the other hand, requires $z \rightarrow 1 / 3$ because of the ill-defined limit $\frac{1}{(x-1)^{3 z-1}}$ as $x \rightarrow 1$ and $z \rightarrow 1 / 3$.

Indeed, the order and directions of the limits may thus play a role, which is evident in the study of the limit $x \rightarrow 1$ and $z \rightarrow 1 / 3$ of $\frac{1}{(x-1)^{3 z-1}}$. However the final result is independent of the way one proceeds in the limit operation. This is because the quantity

$$
\left.\left[\frac{\Gamma(3 z+1)}{23^{3 z+1}}-z \frac{\Gamma(3 z)}{3^{3 z}}+\frac{z}{63^{3 z-1}} \Gamma(3 z)\right]\right|_{z=1 / 3} \equiv 0
$$

The final result is

$$
\lim _{x \rightarrow 1^{-}} \lim _{z \rightarrow 1 / 3} P=-\frac{1}{18} K^{3} I^{(1)^{\prime \prime}}(1)
$$

Therefore, one has that the dimensionless constant is $K=-\left(\frac{18 P}{I^{(1)^{\prime \prime}}(1)}\right)^{1 / 3}$. Because $I^{(1)^{\prime \prime}}(1) \geq 0$ the result is consistent with $n(\omega)>0$ for $\omega<\omega_{c}$ :

$$
n_{\omega}=\left(\frac{18 P}{I^{(1)^{\prime \prime}}(1)}\right)^{1 / 3} \frac{1}{\omega} \log ^{1 / 3}\left(\omega_{c} / \omega\right)
$$

In the other limit $\omega>\omega_{c}$ we can perform the same calculation but changing the integration limit to be consistent

$$
P=-\int_{\omega}^{\infty} K^{3} \omega^{2-3 x}\left(I^{(1)}(x) \log ^{3 z}\left(\omega / \omega_{c}\right)+z I^{(2)}(x) \log ^{3 z-1}\left(\omega / \omega_{c}\right)+I^{(3)}(x, z) \log ^{3 z-2}\left(\omega / \omega_{c}\right)+\ldots\right) d \omega
$$

It is easy to see that the only difference introduced by the change of the integration limits is a single sign:

$$
\int_{\omega}^{\infty} \omega^{2-3 x} \log ^{s}\left(\omega / \omega_{c}\right) d \omega=\frac{\omega_{c}^{3(1-x)}}{3^{1+s}(x-1)^{1+s}} \Gamma\left(1+s, 3(x-1) \log \left(\omega / \omega_{c}\right)\right),
$$

which converges for $x>1$. The final result then is given by

$$
n(\omega)=\left(\frac{18 P}{I^{(1)^{\prime \prime}}(1)}\right)^{1 / 3} \omega^{-1} \log ^{1 / 3}\left(\omega / \omega_{c}\right),
$$

which also gives a positive wave action for $\omega>\omega_{c}$.

The authors acknowledge support from FONDECYT (Chile) grant No. 1130709 (CJ and SR) and FONDECYT grant No. 1150463 (G.D). G.D and C.J. Acknowledge the ECOS-CONICYT C14E04.

[1] K. Hasselmann, J. Fluid Mech. 12 (1962) 481; J. Fluid Mech. 15 (1963) 273.

[2] D.J. Benney and P.G. Saffman, Proc. Roy. Soc. London A 289 (1966) 301.

[3] V. E. Zakharov, V. S. L'vov and G. Falkovich, Kolmogorov Spectra of Turbulence I Springer, Berlin, 1992.

[4] A. Newell, S. Nazarenko, and L. Biven, Physica D 152-153 (2001) 520.

[5] A.C. Newell and B. Rumpf, Annual Review of Fluid Mechanics 43 (2011) 59.

[6] S. Nazarenko, Wave turbulence, Lecture Notes in Physics Vol. 825, Springer Berlin 2011.

[7] V.E. Zakharov, N.N. Filonenko, Dokl. Akad. Nauk SSSR 170 (1966) 1292 [English transl. in Sov. Math. Dokl.].

[8] V.E. Zakharov, Zh. Eksper. Teoret. Fiz. 51 (1966) 686 [English transl. in Sov. Phys. JETP 24 (1967) 455]. 
[9] V.E. Zakharov and N.N. Filonenko, Zh. Prikl. Mekh. I Tekn. Fiz. 5 (1967) 62 [English transl. in J. Appl. Mech. Tech. Phys.].

[10] S. Dyachenko, A. C. Newell, A. Pushkarev and V. E. Zakharov, Physica D 57 (1992) 96.

[11] V.M. Malkin, Phys. Rev. Lett. 76, (1996) 4524.

[12] G. Falkovich and N. Vladimirova, Phys. Rev. E, 91, (2015) 041201(R).

[13] P. A. Hwang, D. W. Wang, E. J. Walsh, W. B. Krabill and R. N. Swift, Journal of Physical Oceanography 30 (2000) 2753.

[14] W. Wright, R. Budakian, and S. Putterman, Phys. Rev. Lett 76 (1996) 4528.

[15] E. Henry, P. Alstrom and M. T. Levinsen, Europhys. Lett., 52 (2000) 27.

[16] M. Yu. Brazhnikov, G.V. Kolmakov, A. A. Levchenko, and L. P. Mezhov-Deglin, JETP Lett. 74 (2001) 583; M. Yu. Brazhnikov, G.V. Kolmakov, and A. A. Levchenko, Sov. Phys. JETP 95 (2002) 447;

M. Yu. Brazhnikov, G. V. Kolmakov, A. A. Levchenko and L. P. Mezhov-Deglin, Europhys. Lett., 58 (2002) 510.

[17] A. N. Pushkarev and V. E. Zakharov, Phys. Rev. Lett. 76 (1996) 3320.

[18] A. I. Dyachenko, A. O. Korotkevich, and V. E. Zakharov, JETP Lett. 77, (2003) 477;

A. I. Dyachenko, A. O. Korotkevich, and V. E. Zakharov, JETP Lett. 77 (2003) 546;

A. I. Dyachenko, A. O. Korotkevich and V. E. Zakharov, Phys. Rev. Lett. 92 (2004) 134501.

[19] G. Düring, C. Josserand, and S. Rica, Phys. Rev. Lett. 97 (2006) 025503.

[20] J.W.S. Rayleigh, The Theory of Sound, Editions Dover (1945).

[21] A. Boudaoud, O. Cadot, B. Odille, and C. Touzé , Phys. Rev. Lett. 100 (2008) 234504.

[22] N. Mordant, Phys. Rev. Lett. 100 (2008) 234505.

[23] P. Cobelli, P. Petitjeans, A. Maurel, V. Pagneux, and N. Mordant, Phys. Rev. Lett. 103 (2009) 204301.

[24] N. Mordant, Eur. Phys. J. B 76 (2010) 537.

[25] O. Cadot, C. Touzé and A. Boudaoud, Phys. Rev. E 82 (2010) 046211.

[26] B. Miquel and N. Mordant, Phys. Rev. Lett. 107 (2011) 034501.

[27] T. Humbert, O. Cadot, G. Düring, C. Josserand, S. Rica and C. Touzé, Euro. Phys. Lett. 102 (2013) 30002.

[28] B. Miquel, A. Alexakis and N. Mordant, Phys. Rev. E 89 (2014) 062925.

[29] T. Humbert, O. Cadot, C. Josserand and C. Touzé, Physica D 316 (2016) 34.

[30] G. Düring, C. Josserand, and S. Rica, Phys. Rev. E 91 (2015) 052916.

[31] C.Touze, S. Bilbao and O. Cadot, 331 (2012) 412.

[32] M. Ducceschi, O. Cadot, C. Touzé and S. Bilbao, Physica D 280-281 (2014) 73.

[33] M.I. Auliel, B. Miquel and N. Mordant, Eur. Phys. J. B 88 (2015) 276.

[34] B. Miquel, A. Alexakis, C. Josserand and N. Mordant, Phys. Rev. Lett. 111 (2013) 054302.

[35] N. Yokoyama and M. Takaoka, Phys. Rev. Lett. 110 (2013) 105501.

[36] N. Yokoyama and M. Takaoka, Phys.Rev. E 89 (2014) 012909.

[37] O. Cadot, A. Boudaoud and C. Touzé, Eur. Phys. J. B 66 (2008) 399.

[38] S. Chibbaro and C. Josserand, Phys. Rev. E 94, 011101(R) (2016). 
[39] Gustavo Düring, "Non-equilibrium dynamics of nonlinear wave systems: Turbulent regime, breakdown and wave condensation", PhD dissertation Université Pierre et Marie Curie, Paris (2010).

[40] A. Föppl, Vorlesungen über technische Mechanik, Bd. 5, Leipzig, (1907) p. 132.

[41] Th. von Kármán, Ency. d. math. Wiss., Bd. IV. 2, II, Leipzig, 1910, §8.

[42] L.D. Landau and E.M. Lifshitz, Theory of Elasticity, Pergamon, New York, 1959.

[43] A.N. Kolmogorov: Dokl. Akad. Nauk SSSR 30 (1941) 299 [ English: Sov. Phys. Dokl.]

[44] Y.V. Lvov and S. Nazarenko, Phys. Rev. E 69, (2004) 066608;

Y. Choi, Y.V. Lvov and S. Nazarenko, Phys. Lett. A 332, (2004) 230;

Y. Choi, Y.V. Lvov and S. Nazarenko, Phys. Rev. E 201, (2005) 121;

Y. Choi, Y.V. Lvov and S. Nazarenko, "Recent developments in fluid dynamics 5 (2004) 225 (Transworld Research Network, Kepala, India).

[45] G. Düring, A. Picozzi and S. Rica, Physica D 238 (2009) 1524.

[46] A.C. Newell, The Closure Problem in a System of Random Gravity Waves, Rev. Geophys. 6 (1968) 1.

[47] D.J. Benney and A.C. Newell, Random Wave Closures, Stud. Appl. Math. 48 (1969) 29.

[48] L. Biven, S. V. Nazarenko and A. C. Newell, Phys. Lett. A 280 (2001) 28.

[49] Lord Rayleigh, Phil. Mag. 49, (1900) 539;

Lord Rayleigh, Nature 72 (1905) 54.

[50] J.H. Jeans, Phil. Mag. 10, (1905) 91;

J.H. Jeans, Phil. Mag. 17, (1909) 229.

[51] R. Jordan and C. Josserand, Phys. Rev. E, 61 (2000) 1527.

[52] S. Bilbao, O. Thomas, C. Touzé and M. Ducceschi, Numerical Methods Partial Differential Equations 31 (2015) 1948.

[53] www.fftw.org

[54] A.C. Newell, "Wave turbulence: a story far from over", Conferece at Ecole Normale Supérieure (2007); http://www.diffusion.ens.fr/index.php?res=conf\&idconf=1831

[55] Th. von Kármán and A.D. Kerr, "Instability of spherical shells subjected to external pressure", Collected works of Th. von Kármán 1952-1963, p. 292 (Technical Editing and Reproduction Ltd, Harford House, London,1975). .

[56] L.H. Donnell, "Beams, plates and shells (McGraw-Hill, New York, 1979).

[57] A.C. Newell and P.J. Aucoin, J.Fluid Mech. 49, pp. 593-609 (1971).

[58] V.S.Lvov, Yu. Lvov, A.C. Newell, and V.Zakharov, Physical Review E. 56, 390-405 (1997)

[59] U. Frisch, S. Kurien, R. Pandit, W. Pauls, S. Sankar Ray, A. Wirth and Ji-Z Zhu, Phys. Rev, Lett. 101 (2008) 144501.

[60] C. Leith, Phys. of Fluids 10 (1967) 1409. 


\section{Highlights}

- Wave turbulence

- Elastic plates

- Kinetic equation

- Numerical simulations 